BNL -51950

(Particle Accelerators

DE86 006761

\title{
HIGH ENERGY PHYSICS IN THE UNIIED STATES
}

M. Month

October 16. 1985

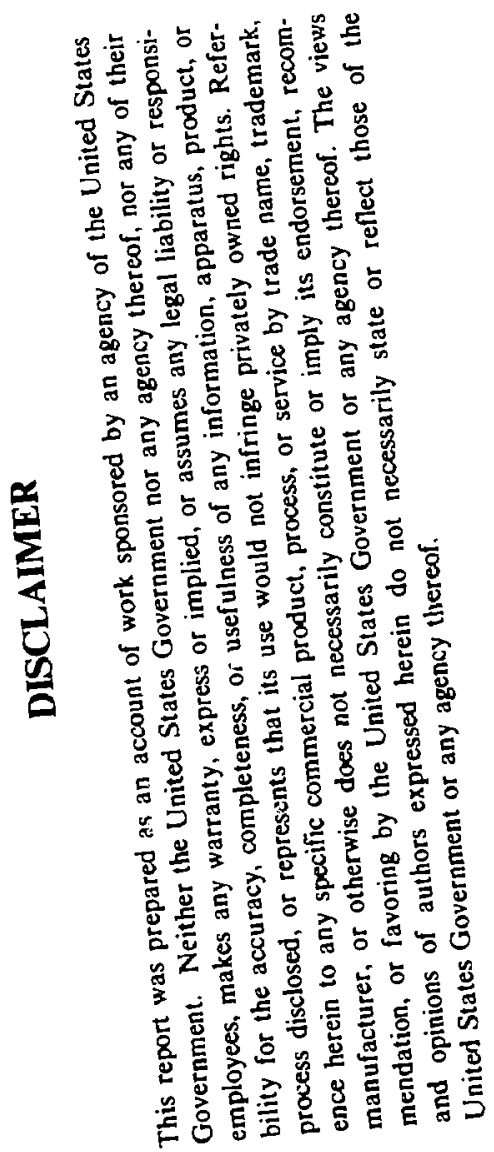

\section{ALTERNATING GRADIENT SYNCHROTRON DEPARTMENT}

BROOKHAVEN NATIONAL LABORATORY UPTON, LONG ISLAND, NEW YORK 11973 


\section{DISCLAIMER}

This report was prepared as an account of work sponsored by an agency of the Lnited States Govemment. Neither the United States Govemment nor any agency thereof. nor any of their employeeg. nor any of their contractors. subcontractors. or their employees, makes any warranty, express or implied. or assumes any legal liability or responsibility for the accuracy, completeness, or usefulness of any information. apparatus. product. or process disclosed. or represents that its use would not infringe privately owned rights. Reference herein to any specific commerctal product. process. or gervice by trade name. trademark. manufacturer. or otherwise. does not necessanly constitute or imply its endorsement. recommendation, or tavoring by the Lnited States

Government or any agency, contractor or subcontractor thereof. The views and opinions of authors expressed herein do not necessarily state or reflect those of the United States Government or any agency, contractor or subcontractor thereot.

Printed in the United States of America Available from

National Technical Information Service

U.S. Department of Commerce

5285 Port Royal Road

Springfield, VA 22161

NTIS price codes:

Printed Copy: AC:5: Microfiche Copy: A01 
HIGH ENERGY PHYSICS IN THE UNITED STATES*

\author{
Melvin Month \\ Brookhaven National Laboratory \\ Upton, NY 11973
}

TABLE OF CONTENTS

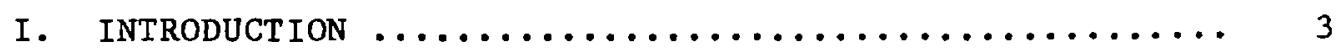

A. Overview of the Field ................... 3

B. Planning for the U.S. High Energy Physics Program. 4

C. Goals and Status of High Energy Physics ......... 8

D. Evolution of Accelerator and Detector Facilities.. 14

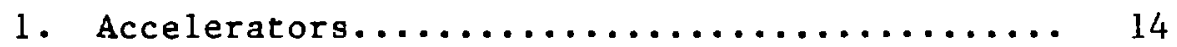

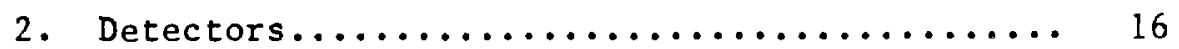

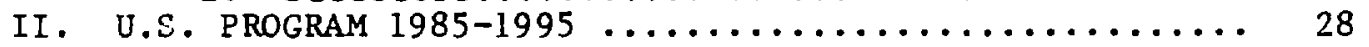

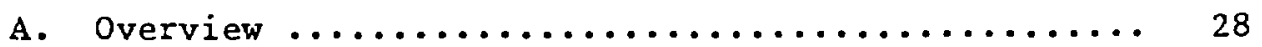

B. Details of the Program ................... 29

1. Brookhaven National Laboratory (BNL) ....... 33

2. Stanford Linear Accelerator Center (SLAC) .... 34

a. SLAC Fixed-Target Program ............ 34

b. PEP Program at SLAC .............. 35

c. SPEAR Program at SLAC .............. 35

d. SLAC Linear Collider (SLC) ........... 36

3. Fermi National Accelerator Laboratory

(Fermilab) ........................ 38

a. TEVATRON II Program at Fermilab ....... 39

b. TEVATRon I Program at Fermilab ......... 40

4. Cornel1 Laboratory for Nuclear Studies ...... 42

5. Non-Accelerator Program .............. 43

C. University-Based Research ................ 44

III. INTERNATIONAL COOPERATION IN HIGH ENERGY PHYSICS .... 48

A. General ............................. 48

B. The European Program ................... 52

1. CERN ................................. 52

a. Fixed-Target Operation of the Super

Proton Synchrotron (SPS) ............. 52

b. The SPS Proton-Antiproton Collider ...... 52

c. Large Electron-Positron Facility (LEP) ... 52

d. The Next Step at CERN .............. 52

*The basis for this paper was a lecture given by W.A. Wallenmeyer (Director of the Division of High High Energy Physics of the Department of Energy) at the 1983 Summer School at BNL. The author of this paper would like to express his appreciation to Dr. Wallenmeyer for his encouragement and support and to the program office staff at DOE for their cooperation and help. The actual material was accumulated from the DOE congressional budget writeup and briefings, from descriptive information prepared by the national laboratories, from various surveys and reports commissioned by the DOE and its scientific advisory body HEPAP, and from other documents. 


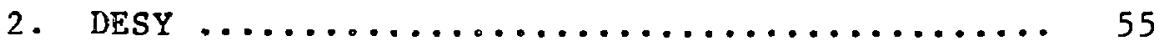

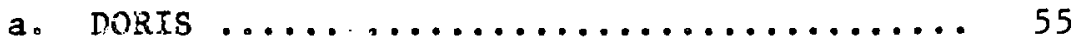

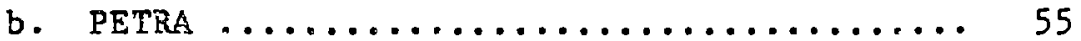

c. HERA ............................ 55

C. The Japanese Program .................. 56

D. The U.S.S.K. Program ..................... 57

1. Institute for High Energy Physics (IHEP),

Serpukhov ..................... 57

2. Institute for Nucsear Physics (INP),

Novesibirsk ............................ 58

3. Joint Institute for Nuclear Research (JINR),

Dubra ............................ 58

E. The Program of the People's Republic of China .... 59

IV. NEW FACILITY NEEDS FOR THE U.S. HIGH ENERGY PHYSICS

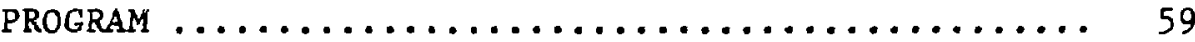

A. The Next step ........................... 59

B. Accelerator $R$ and $D$ in Superconductivity........ 64

C. Longer Range .......................... 66

D. Advanced Technology Research and Development .... 67

E. Funding and the Transition to the SSC .......... 69

1. Evolution of the Research Program .......... 69

2. Funding Required for the High Energy

Physics Program .................. 70

3. Scientific Manpower .................. 71 
HIGH ENERGY PHYSICS IN THE UNITED STATES

\author{
Melvin Month \\ Brookhaven National Laboratory. Upton, NY 11973
}

I. INTRODUCTION

A. Overview of the Field

High energy physics is the field of basic research which addresses the most fundamental questions concerning the nature of the physical universe, i.e., the basic nature of matter, energy, space, and time. Its objective is to find the fundamental constituents of matter (the elementary particles) and the forces that act between them. Recent developments in experimental and theoretical physics point the way to an increasing understanding of the basic structure of matter and to an overall synthesis encompasing all the forces observed in nature.

Exploration of the ultimate constituents of matter requires two essential tools: particle beams of high enough energy and intensity to probe the structure within the nucleons, and detectors sensitive and complex enough to detect and decipher that structure.

The particle beams are generared by complex and large accelerators of various types, including linear accelerators, circular accelerators (synchrotrons), and colliding beam machines. The more fundamen-tal the structure to be probed, the higher are the energies needed; therefore, attempts to probe deeper into the structure of matter require new accelerato: capabilities and often new accelerator technologies. In resent years, devlopments in superconducting acceierator magnets and in colliding beam technology have provided the base for the next step in major facility development and construction.

Accelerating particles and bringing them into collision with targets and other beams is only half the task. The other half is to observe and distinguish the particles that emerge from these collisions with particle detectors. Much ingenuity has gone into the conception, development, and fabrication of these devices that can simultaneously register the passage of many subatomic particles traveling at essentially the speed of light, recognize their nature, and measure their energy and other properties.

Particle detectors have come to have a highly sophisticated set of capabilities due to the rapid development of electronics and other technology developments in recent years. Conversely, $R$ and $D$ to meet detector requirements has contributed to developments in a variety of technologies. This process, in response to the increasingly stringent requirements of experimentation at higher energies, has resulted in great improvements in precision and sensitivity and given rise to the modern detector, a large, complex multicomponent instrument.

The increased detector capability coupled with the higher energy and intensity of accelerator beams has resulted in massive amounts of 
data for analysis, which is. done with powerful computers. Some computers are integrated into the detectors and are used to control the apparatus and to analyze data in real time in order to provide rapid feedback of results to guide the conduct of the experiment. Theoretical physicists and accelerator physicists have also come to rely on computers for the complex calculations needed to solve forefront theoretical problems and to simulate the properties of accelerators under design.

\section{B. Planning for the U.S. High Energy Physics Program}

High energy physics research is dependent on large complex particle accelerators, colliding beans, and detector facilities, and requires long lead times for planning and implementing intricate experiments and for designing and constructing advanced facilities. Typically, the time from the original concept for an experiment to the publication of results is 3 to 6 years and the time from conceptual design to operation for a major facility is 5 to 10 years or more. In an endeavor with such long lead times effective long-range planning is essential. High energy. physics has a long record of efficient longrange planning. Since 1967 planning for the U.S. High Energy Physics Program has benefited substantially from advice from the High Energy Physics Advisory Panel (HEPAP) and its subpanels. Figure 1 indicates the role of the Department of Energy (DOE) as the lead agency responsible for this Program.

Institutionally, the progran structure has at its core the large national accelerator laboratories (BNL, Fermilab, and SLAC managed by DOE and Cornell by NSF). Experimental support for the High Energy Physics Program is provided by 120 groups from 64 universities and laboratories with DOE funding and by 90 groups from 52 institutions with NSF funding; theoretical expertise derives form 57 DOE funded

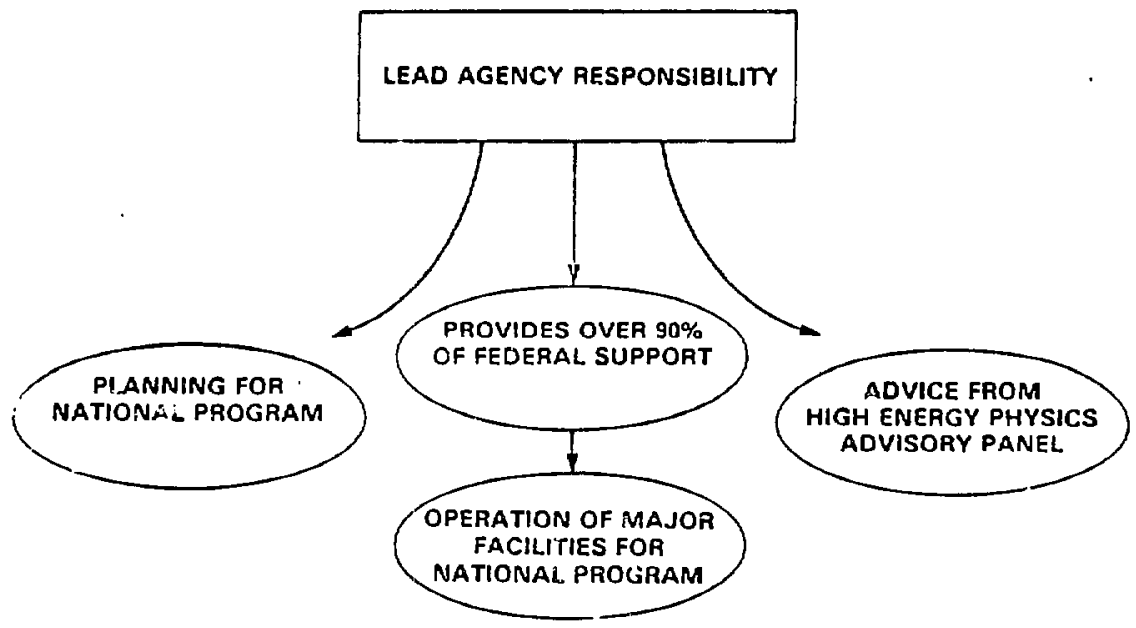

Figure 1. The role of the Department of Energy as the lead agency responsible for the U.S. High Energy Physics Program. The other U.S. government agency responsible for support to High Energy Physics is the National Science Foundation (NSF). 
universities and 1 aboratories and 47 NSF funded instltutions. The DOE management of the program is governed by a program philosophy which can be summarized as follows:

- Ideas and froposals are generated by scientists in the field.

- The agency, with input from the field, establishes policy, plans, and budgets.

- The agency provides funding allocations and general guidance to the field.

- The laboratory management or university principal investigator is entrusted with the responsibility for the day-to-day detailed managenent of the program.

- The agency reviews and monitors progress and takes corrective action where appropriate.

The annual budget process by which funds get allocated is a rather elaborate one, beginning with proposals from the field and culminating in a funding decision process involving the Congress, the Office of Managment and Budget (OMB), and the Department of Energy (DOE).

Within the DOE, the Division of High Energy Physics (DHEP) first puts together a proposed High Energy Physics budget. This budget then moves to the Office of High Energy and Nuclear Physics (OHENP) where it gets folded in with the Nuclear Physics Budget. As the budget proceeds up the organizational ladder, there is negotiation and budget fitting and reworking. After OHENP, the office of Energy Research (OER) has a budget which includes Fusion and Basic Energy Sciences. Finally, the budget goes through the Undersecretary of Energy and the Comptroller and becomes a total DOE Budget including other pirts of the Department's concerns such as Nuclear Energy and Nuclear Weapons. The OMB acts for the President and pulls together from all the agencies the President's Budget, which is then sent to the Congress. The House and the Senate each act on the President's Budget through three committees: the Budget Committee, the Authorization Committee, and the Appropriation Committee. Hearings are held before subcomittees of each of these in both the House and Senate. Coming out of the hearings, House and Senate bills provide budget figures. If they differ there is a House and Senate conference, and from this conference comes a joint House and Senate bill. The bill returns to the President and with his signature it becomes $1 \mathrm{aw}$. The bill then returns down the line essentially the same way it went up, and apportionment is made at each level.

The government operates on a fiscal year basis. The fiscal year begins on October 1 . The budget process begins with the receipt of requests from the field about 18 months before the beginning of the fiscal year. The DOE then sends its request to the OMB about 13 months before the fiscal year begins. Sometime in January, the President's Budget is released and sent to Congress about 9 months before the fiscal year starts. Under ideal circumstances, Congress will return the budget bill to the President about 3 months before October 1; sometimes this doesn't happen, and no budget bill is passed into law by the beginning of the fiscal year. Since government operations must continue, the government then proceeds on the basis of a continuing 
resolution, a temporary bill that Congress must pass just for this purpose.

The steps in the DOE process are reviewed in Table I, and the annual budget process is outlined in Figure 2. An example of a High Energy Physics Budget allocation, including figures for the FY 1986 President's Budget, is shown in Table II.

Planning for the DOE High Energy Physics Program during FY 1979 -FY 1982 was based on a long-range funding plan agreed to in early 1978 by the DOE and the Office of Management and Budget (OMB). The objectives were to maintain a productive and viable High Energy Physics Program in the U.S. effectively utilizing three accelerator centers (Brookhaven, Fermilab, and SLAC); to maintain U.S. world leadership in the field; to permit construction of new facilities as required: and to accomplish these goals within an approximately constant budget level of $\$ 300 \mathrm{mill}$ ion per vear in FY 1979 dollars.

Since the time of the DOE/OMB agreement, many profound scientific and technical insights have been gained. Major physics developments include the discovery at $C E R N$ of the $W$ and $Z$ particles, which are crucial to theories that unify the weak and electromagnetic forces; the emergence of the theory of quantum chromodynamics ( $Q C D)$, which describes the strong force in terms of the interactions between quarks and gluons; and progress toward grand unified theories which link the strong force with the electroweak force in one comprehensive framework. Breakthroughs in understanding are expected to occur in the mass region where elementary subnuclear constituents interact with energies of a few $\mathrm{TeV}$ ( $10^{12}$ electron volts). Many theoretical approaches point to the existence of hitherto unobserved forms of matter in this mass region. The discovery of such new forms of matter would have a profound impact on our understanding of the underlying symmetries of nature and the mechanism for generating the masses of elementary particles.

Major technology and facility developments have also occurred. These include the successful operation at Fermilab of the Energy Saver, the world's first high energy accelerator using superconducting magnets, the TEV II fixed-target project, and the TeV I collider project; the construction of the SLAC SLC and the CERN LEP electronpositron collider projects; the successful achievement of adequate 1 uminosity in the $p \bar{p}$ collider at CERN; and the decision not to proceed with the CBA project at Brookhaven. The success of the Energv Saver, together with $R$ and $D$ progress in the CBA superconducting magnet program, give confidence that very large accelerators using superconducting magnets are feasible. In view of these developments it was prudent that the goals and directions of the U.S. High Energy Physics Program be reassessed.

First steps toward a review and reevaluation of the U.S. High Energy Physics Program plan were taken in mid-1981: A HEPAP Subpanel on Long-Range Planning reviewed and evaluated in depth the high energy physics facilities currently in operation, those under construction, and those proposed for construction. A report with long-range planning recommendations at two assumed budget levels was completed in January 1982. In 1983, a HEPAP Subpanel on New Facilities conducted an in-depth review of the scientific requirements and opportunities 


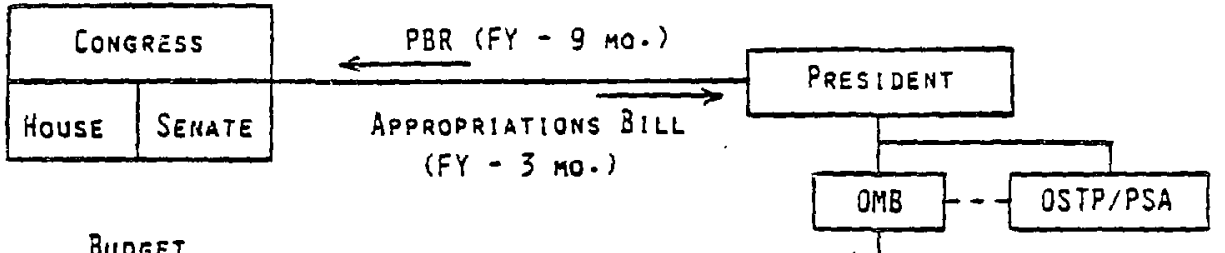

BUDGET

Authorization

DOE REQUEST

Appropriation

TASLE I (FY - 15 mo.)

STEPS IN DOE BUDGET PRICESS

INPUT FROM LabORATORY AND UNIVERSITY

DIVISION OF HIGH ENERGY PHYSICS DFFILE OF HIGH ENERGY AND NUCLEAR PHYSICS FORMULATES BUDGET

DFFICE OF ENERGY RESEARCY REYIEX

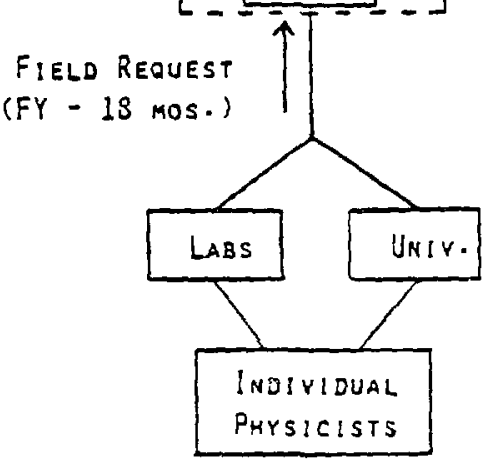

Figure 2.

U.S. DEPARTMENT OF ENERGY Annual budget process.

OFFICE OF MANAGEIENT AND BUDGET

REVIEW

PRESIDENT'S BUDGET TO CONGRESS

HOUSE ACTION

SENATE ACTIOH

CONFERENCE

CONGRESSIONAL BILL

BILL

PRESIDENT

FOR SIGMATURE

OFFICE OF MHWAGEMENT AND BUDGET

APPORTIONMENT

U.S. DEPARTMENT OF EHERGY

ALLOCATION 
Table II. An example of a High Energy Physics Budget $B / A=$ Budget Authorization (authorization to use funds) $B / O=$ Budget Outlays (funds that can be costed)

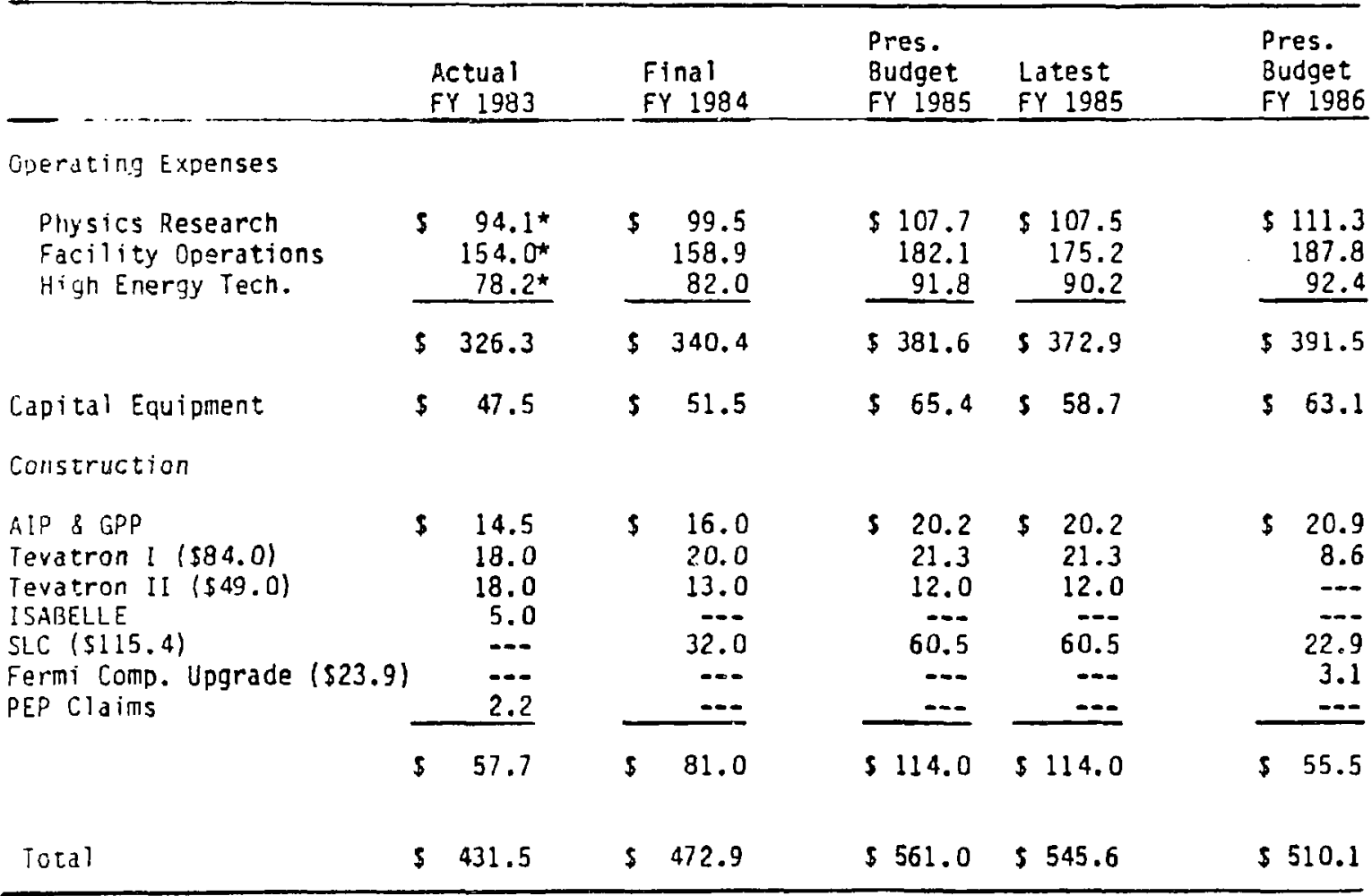

$\star^{B / O}$ given here. Because of GSO adjustments between subprograms, $8 / 0$ gives indication of program activity.

for a forefront U.S. program in the next five to ten years. This report was unanimously endorsed by HEPAP and was published in July 1983. More recently, a HEPAP in-depth examination of the entire U.S. program was made. This study culminated in a meeting at Berkeley Springs, WV, June 2-8, 1985. Its conclusions are detailed in Report of the 1985 High Energy Physics Advisory Panel Stu'y of the U.S. High Energy Physics Program 1985-1995, published by the U.S. Department of Energy Division of High Energy Physics. It reaffirmed the highest priority for the SSC, identified top priority elements in the present Program, and considered the transition to the SSC.

These reports provided valuable input on high energy physics research and facility status, future facility needs, and program priorities to the HEPAP, which reviewed the reports and forwarded its advice and recommendations to the Department of Energy.

C. Goals and Status of High Energy Physics

The goal of high energy physics is to find the ultimate constituents of matter and the forces acting between them. The purpose of the program is to examine the transformations and interactions among the ultimate constituents of matter, to search for new fundamental 1 aws of nature, and to seek a better understanding of the established laws of nature. Research into the inner structure of matter has proceeded 
From the level of molecules to atoms, to nuclei, to nucleons, and recently to constituents inside the nucleon called "quarks." Quarks and leptons (electrons, neutrinos, and massive electron-like particles) form simple families and are the most basic constituents of matter so far understood (Figure 3). High energy physics deals with distance scales (Figure 4) of $<10^{-13} \mathrm{~cm}$. Such deep penetration into matter can be accomplished unly by using particle beams of very high energy (and thus very short wave-length), available from today's giant particle accelerators.

As newer accelerators have probed to smaller distances in matter, a theory has evolved to account for the multitude of observations which has become so widely accepted that it is called the "standard picture." The elemental constituents of matter are currently viewed as six quarks and six leptons, which have distinct physical properties but no internal structure and no size. Quarks combine under the strong force, a residue of which binds protons and neutrons into atomic nuclei and is responsible for fusion processes that power the

\section{BASIC CONSTITUENTS OF MATTER}

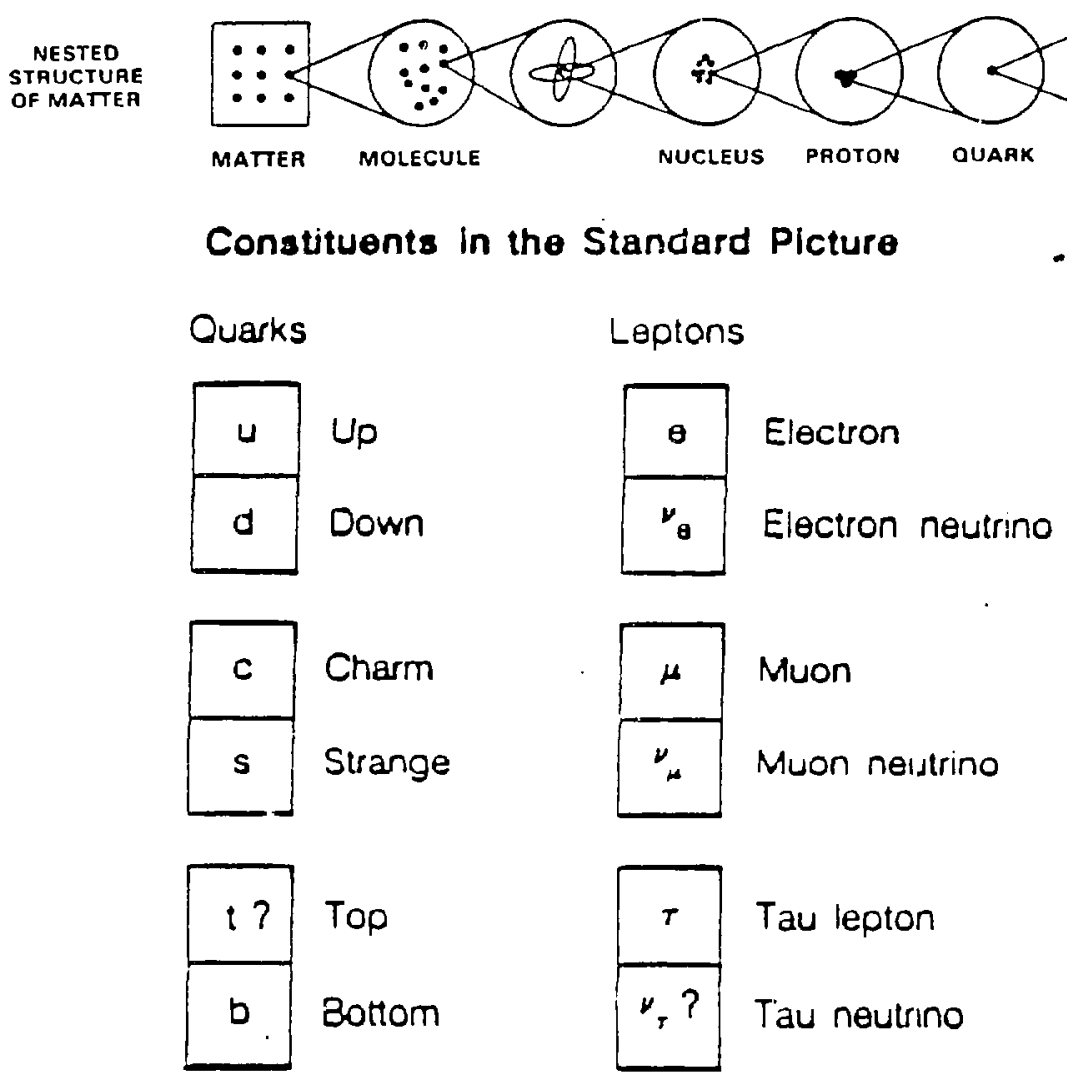

Figure 3. Successive levels in the nested structure of matter discovered through more than a century of research at the "high energy" frontier, and the elementary constituents of matter as indicated by the standard picture, grouped into families of leptons and quarks and into three generations. 


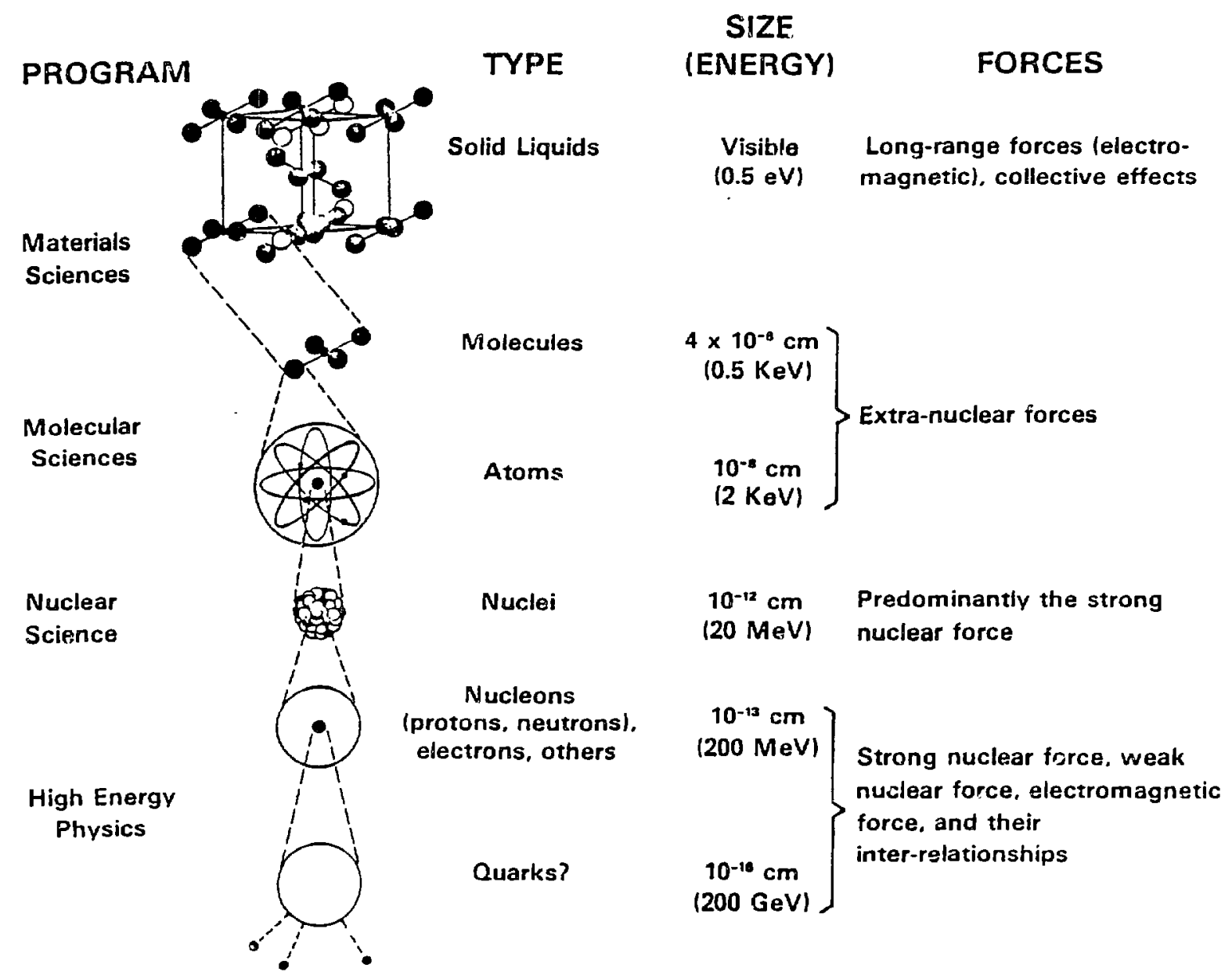

Figure 4a. Typical dimensions of matter for several disciplines ranging from materials sciences to high energy physics at the subatomic scale.

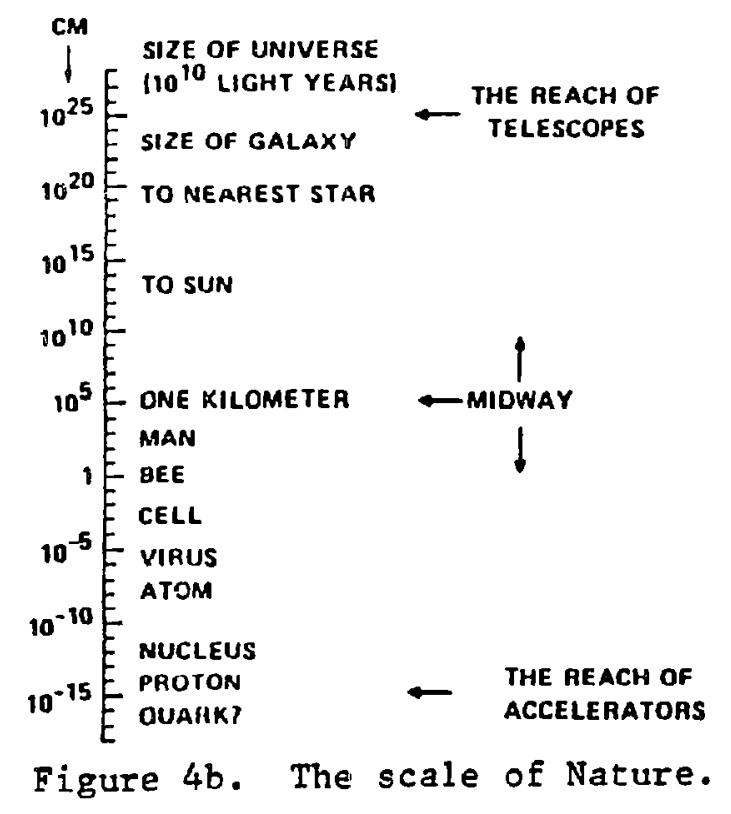


stars; leptons are unaffected by this strong force but are subject, as are the quarks, to the electromagnetic force that binds leptons (i.e. electrons) to nuclear matter to form atoms and to a weak force that generates transitions among quarks and leptons and manifests itself as radioactivity. Gravity is a fourth force, relatively so weak that it would require the combined effect of an enormous number of particles to make it measurable at the scale of distance sc far studied (see Table III). Physicists hope ultimately to unify and so describe all fundamental forces by a single unified theory (Einstein's dream).

This standard picture has brought high energy physics to a turning point. During the last decade, work in the gauge theory of particle interactions has shown how the fundamental forces of nature might be unified. The electromagnetic force has been unified with the weak nuclear interaction even though these two forces differ in strength by a factor of narly 100,000. This has encouraged the hope that unification of all the fundamental forces may be within reach during this century (Figure 5). Gauge theory now dominates nearly all phases of high energy physics, and the decision to perform new experiments is often based on their relevance for testing its predictions. The potential applications for gauge theory extend far beyond elemericary particle physics, to areas as diverse as condensed matter physics, nonlinear wave phenomena, atid even pure mathematics.

The primary principle of modern gauge theory is that forces have a property designated "local gauge invariance," a mathematical symetry such that the various basic forces have a comun mathematical structure so that the different forces appear to be manifestations of a greater unified whole. A second principle is that a phenomenon called "spontaneous symmetry breaking" generates the large differences between the strong, weak nuclear, and electromagnetic forces. This mechanism generates the masses of the elementary particles which reflect the differing natures of the forces observed; the photon, carrier of the electromagnetic force, is massless, whereas its analogues, the vector bosons ( $W^{ \pm}$and $Z^{\circ}$ ), which carry the weak nuclear force, are quite massive. The recent discovery of these bosons at CERN corifirms their huge mass--almost 100 times that of the proton--in agreement with the prediction.

To understand the generation of the masses of vector bosons and other elementary particles requires that one understand how spontaneous symetry breaking is manifested in nature. A likely manifestation is an as yet unseen type of subnuclear particle--one with no intrinsic spin (as contrasted with quarks and leptons, which have internal spins of half units, and the carriers of the strong, weak, and electromagnetic forces, which have a spin of one unit). This particle, called the Higgs boson, is predicted by gauge theory. A primary goal of research in the next decade is to discover the Higgs particle or some manifestation of the mechanisin for spontaneous symetry breaking. This could lead to the discovery of a class of matter with fundamentally new properties which would complement the quarks, leptons, photons, and other gauge bosons found at the smallest scale of distance now attainable. This would have far-reaching intellectual and scientific consequences. 
Table III The characteristics of the four basic forces

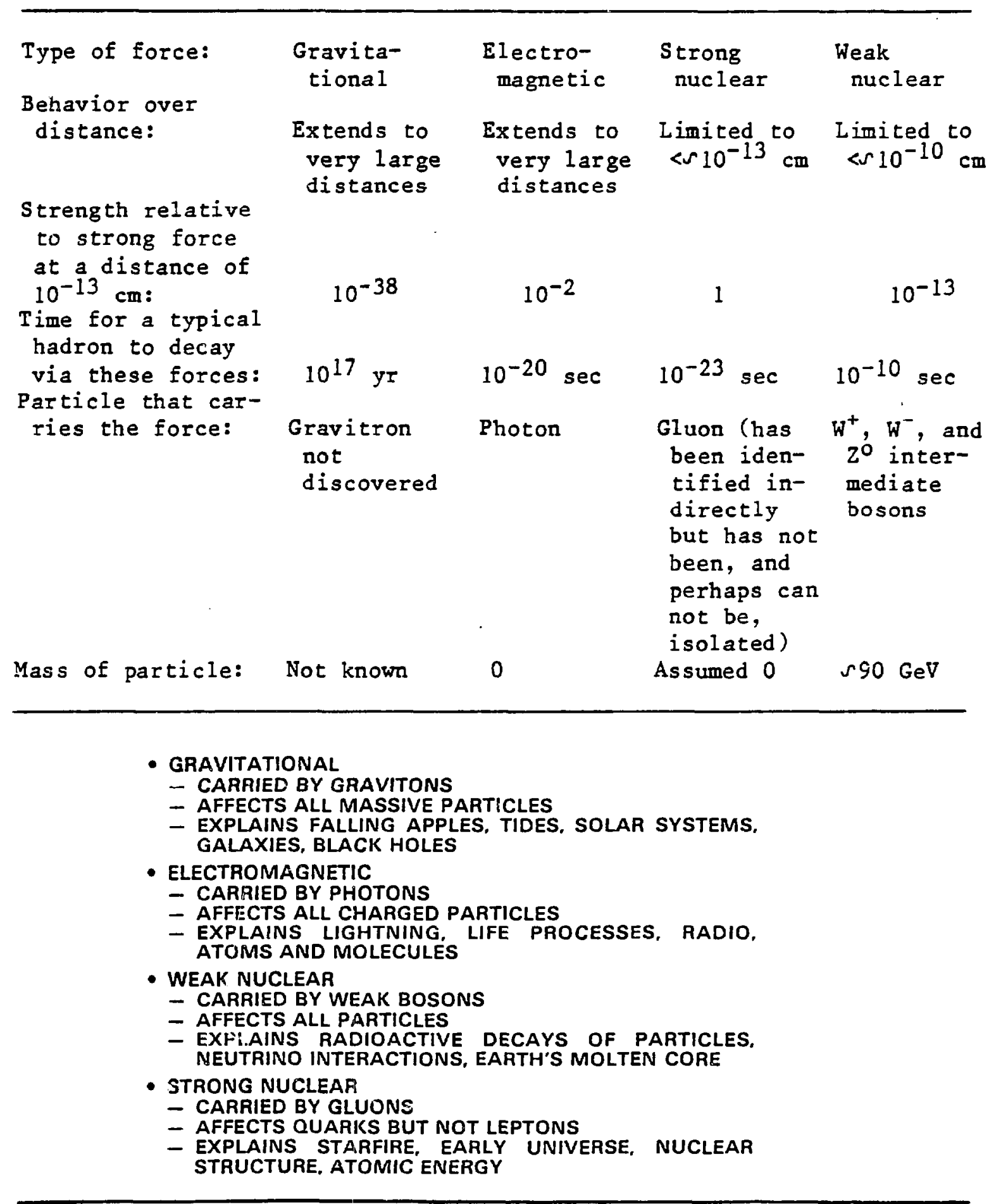




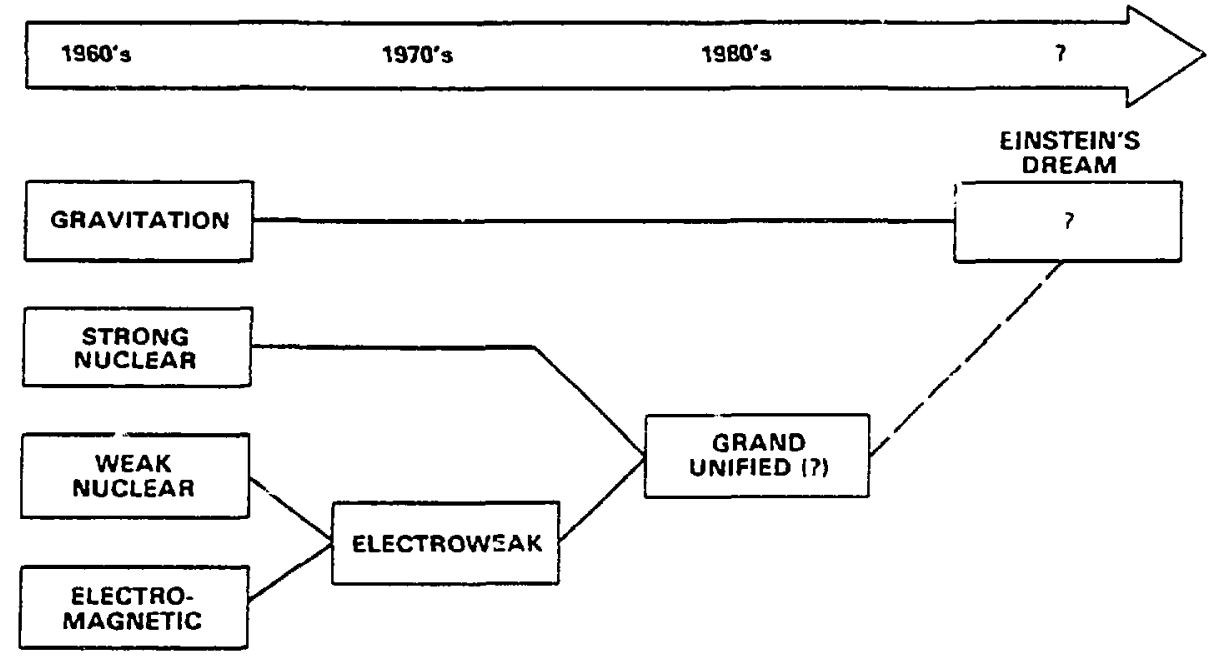

Figure 5. The process of unification of the basic forces of nature over the past quarter century.

All current theories predict fundamental new phenomena at mass values of a few $\mathrm{TeV}$ or less. The need to probe the mass region up to a few $\mathrm{TeV}$ sets the basic requirements for beam energy aid luminosity (beam collision rate) for the next step in the construction of high energy accelerator and colliding beam facilities. The overall priority in high energy physics for exploring the few- $\mathrm{TeV}$ mass region arises from the critical question of spontaneous symetry breaking and the strong expectation that major breakthroughs can be made when the right energy range is probed.

Theory has pushed even further into the unknown and several trial models of "grand unification" unify the description of quark and lepton matter at higher energies and predict that quarks may turn into leptors and vice-versa. This implies the exact equality of lepton and nucleon electric charges and leads to the prediction that protons, long thought to be stable, will be found to decay. These models further indicate that magnetic monopoles, trillions of times heavier than protons, may exist; and some predict that matter may change to antimatter, which implies that neutrinos, previously thought to be massless, may have very small masses that have escaped detection. Finally, these models may provide an approach to understanding why certain microscopic processes do not proceed forward and backward rxactly reversibly (CP violation). Many of the predictions are subject to test in experiments already in progress or being designed for facilities now under construction.

A primary goal of the next decade is therefore to continue unifying the four basic forces of nature. Present theories are expected to be developed and extended, and their predictions will be experimentally tested at facilities existing and under construction. Early in the next decade a new colliding beam accelerator giving access to the $\mathrm{TeV}$ mass region will provide insight into the breaking of gauge symetry and hence allow physicists to formulate the scientific foundation of higher-order unification schemes. Research in high 
energy physics may help to ultimately clarify how the universe evolved srom the "big bang" to its present state and how it will continue to evolve. The study of proton decay and $C P$ violation miy explain why the unirerse evolved almost exclusively into matter rather than equally into mater and antimatter. The observation of a neutrino with nonzero mass may indicate whether the universe will expand forever or contract eventually to another fireball. The high energies achieved by accelerators allow the small-scale simulation of temperature conditions thought to have existed a fraction of a second after the big bang.

Other directions for high energy physics research include the search for new or more elementary constituents more massive than any now known; the search for structure within constituents now considered elementary; the study of the fundamental forces at a distance smaller than that accessible with existing facilities; and the role of the gravitational force in the small-distance domain of the elementary particles. The most significant discoveries could be totally unexpected. High energy physics research, as it probes deeper into matter has in the past seen many of the surprises nature has to offer, and in the next step--to higher energies and smaller subnuslear distances--we can only wonder what nature has in store for us.

\section{Evolution of Accelerator and Detector Facilities}

\section{Accelerators}

In this century the push to identify the ultinate constituents of matter and the basic forces that bind them has led to the consituction of large and powerful research instruments such as the high energy accelerators at CERN (near Geneva), at DESY (near Hamburg, West Germany), at Stanford University (SLAC; in California), at Brookhaven National Laboratory (near New York City), and at Fermilab (near Chicago). Since the $1940 \mathrm{~s}$ : significant discoveries about nuclear and subnuclear structure have been closely related to the availability of increasingly more powerful accelerators providing particles of higher and higher energies. The energy growth of accelerators between 1930 and the present is shown in Figure 6 .

The smaller the domain to be studied, the more powerful must be the instrument. The tremendous binding forces within and between subatomic particles require the high energy of a particle accelerator to split the particles into new components, which can then be observed individually and as they interact. Pirt of the immense energy is converted into matter, producing a profusion of particles of varying degrees of stability.

Particle accelerators can be linear accelerators (such as the 30-GeV electron linac at SLAC) in which the particles go through only once, reaching maximum energy at the end, or circular synchrotrons (such as the $800-\mathrm{GeV}$ Tevatron at Fermilab or the $30-\mathrm{GeV}$ Alternating Gradient Synchrotron at Brookhaven), in which the particles travel around a circular path, gaining energy with each revolution. Electrical energy is used to accelerate the particles; whereas in synchro- 


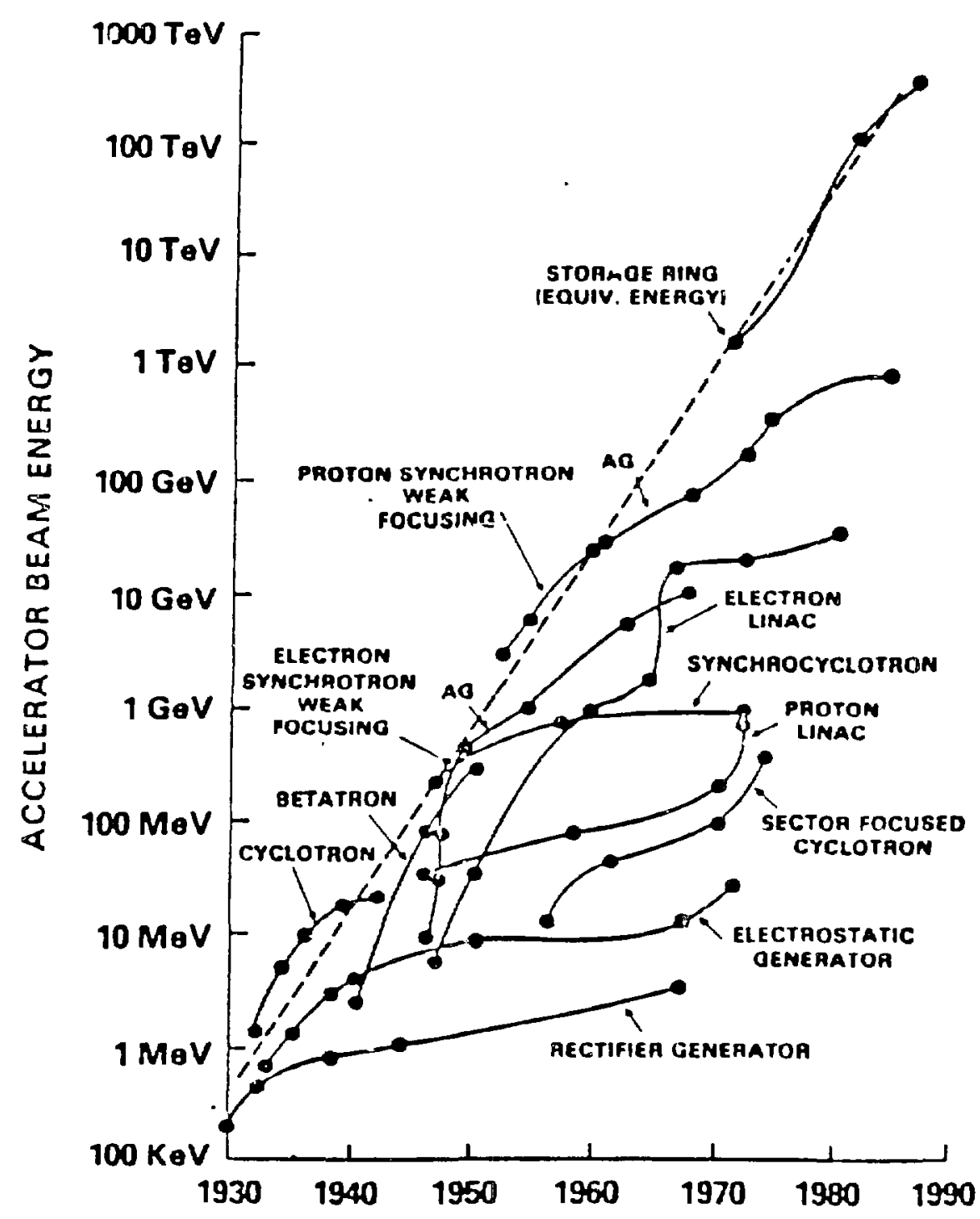

Figure 6. Energy growth of accelerators and storage rings.

trons powerful magnets keep the particles in the circular path and focus them into a narrow beam.

During the 1960 s and 1970 s major advances in accelerator technology (the accumulation and long-time storage of intense beams of hadrons and leptons) made possible the head-on collision of high energy particle beams in circular accelerators. This dramatically increased the energy available for initiating new forms of elementary processes and probing sualler subnuclear distances. For example, in order to reach the available energy produced in a head-on collision between two 1000-GeV protons, a proton colliding with a fixed target proton would have to have an energy of $2 \mathrm{million} \mathrm{GeV}$, requiring an accelerator with a diameter of $\$ 8000$ miles.

In addition to particle beam energy, another basic accelerator parameter is the beam intensity, or the associated collider luminosity. The luminosity of a collider determines the rate at which the 
particles in one beam colifide with those in the other beam. The greater the beam intensity and density, the greater the luminosity. Increased density for a given bean intensity is achieved by focusing the beams to reduce their cross section and by radiofrequency systems to reduce the bunch length. Improved focusing techniques have achieved luminosities high enough to permit detailed investigation of interesting and rare reactions.

Colliding beam systems have the advantage over fixed-target machines of high reaction energy for a given beam energy but the disadvantage that interactions amenable to study are restricted to collisions between the stored parsicles. In addition, positron and electron beams colliding in a storage ring annihilate each other and provide a simple initial state of pure energy, uncomplicated by strong interactions. On the other hand, proton-proton and proton-antiproton colliders can provide the highest reaction energies, which would be prohibitively expensive if obtained by other means, but pay a price in that the variety of collisions that can be studjed is restricted to interactions of particles with complex internal structure. However, by storing other particles and : ath suitable modifications to the collision configuration, colliders could achieve electron-proton (as in the HERA project at DESY) and deuteron-proton collisions and even collisions of heavy nuc: $\geq i$.

A beam of arce erated particles colliding with a fixed target, although not attaining the high reaction energy of colliding beams, has the virtue of providing a large interaction rate and a large variety of secondary particle beams. Fron the products of the primary collision, intense secondary beams of various types of strongly interacting particles (pions, kaons, neutrons, etc.) can be produced to allow many differerir approaches to the study of interactions. In this way, the propertifs of hadrons other than the proton can be directly explored. It is also possible to produce secondary beams of neutrinos, electrons, auons, photons and other particles, which are valuable for studying weak and electromagnetic interactions of hadrons and the properties of :heir internal constituents.

Schematics of these modern high energy physics accelerator facilities, emphasizing their special features, are shown in Figure 7 , including fixed target, $e^{+} e^{-}, p p$ and $p \bar{p}$ facilities.

\section{Detectors}

As their energy has increased, particle accelerators and colliders not only have increased in size and complexity but also have come to depend on large and intricate detectors to record the pattern of particles emanating from the collisions, often in time intervals of billionths of a second. These detectors, consisting of a number of devices (bubble chambers, Cerenkov counters, proportional wire chanbers, drift chambers, charge coupled detectors, time projection chambers, scintillation counters, etc.) require advanced technology and high precision.

Detectors extract the physics from particle collisions by making "visible" the products of the interactions of the high energy particles. Elementary particles cannot be seen directly; their path 
Figure 7A. Froton beam colliding with fixed-target accelerator. Available reaction energy $s \sqrt{2 \times \text { heam energy. }}$
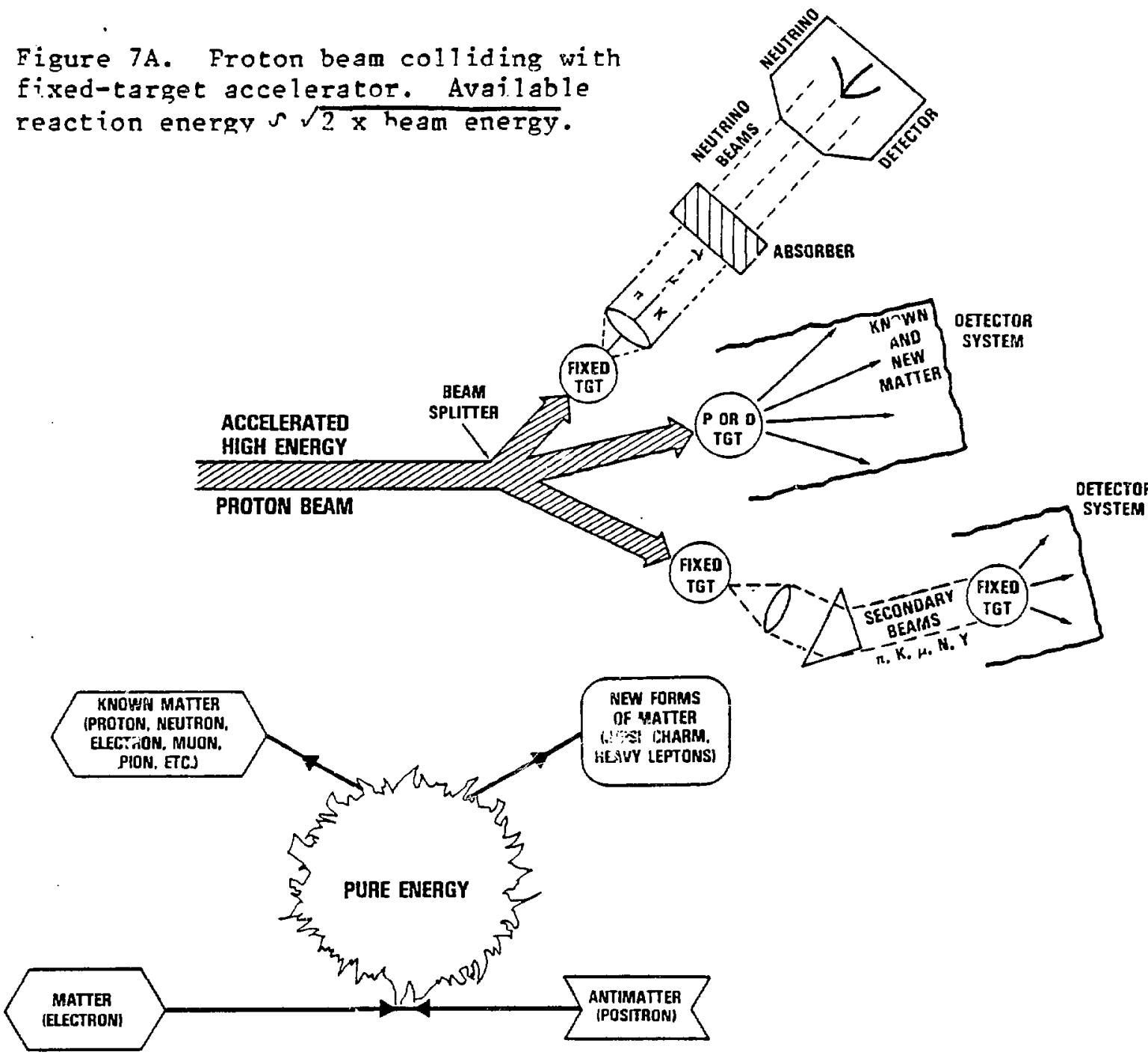

Figure 7B. Positron-electron colliding beam system. Matter-energy conversion and new particle production.

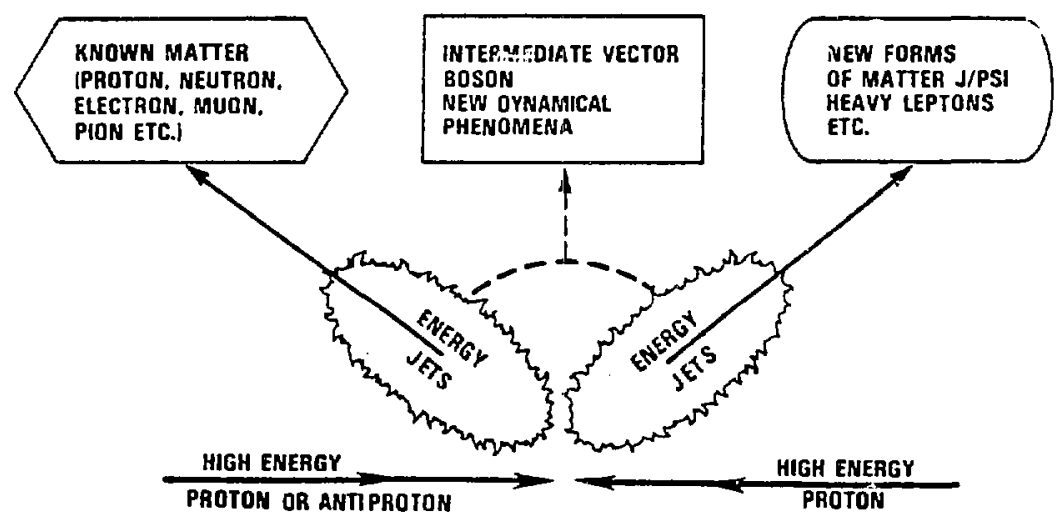

Figure 7C. Hadron-hadron colliding beam system. High available reaction energy $=2 \mathrm{x}$ beam energy. 
and energy as they come out of an accelerator or out of a collison must be determined indirectly. It is also important to identify the typo of particle: is it an electron, muon, proton, photon and so forth. Thus detectors are often very complex. The strong interest in rare processes (such as $W$ and $Z$ production in the recent CERN experiments), and the need to completely characterize events, have led to the development of detectors sensitive to almost the total solid angle. At all accelerators, but particularly at particle colliders, it is essential to provide detectors caparle of making the fullest use of the particle beams. A wide range of detectors exists including the small but often very sophisticated instruments designed for fixedtarget work, the very large detectors used for recording rare events such as the interactions of neutrinos or the decays of nucleons, and the large collider detectors that provide almost complete angular coverage and characterization of the interacations occurring in these machines. This latter class of collider detectors is among the most costly and demanding. Their technological problews and soluticns are, in large part, shared with the other clasjes of detectors.

The MARK I detector first used at SPEAR in 1972 was the first electronic detector for a particle collider with close to full angular coverage and with a magn ic icield to provide momentum and energy measurements for charged paricicles. Two riews of the MARK I are shown in Figure 8, and a reconstructed "picture" of a $\mathrm{J} / \psi$ (psi) event is shown in Figure 9. This detector was sophisticated for its era, allowing the discovery of the $\psi$, the tau lepton, and charmed particles.

A bare decade later comparably important results have begun to flow from the detectors at the CERN proton-antiproton collider with the discovery of the $Z$ and $W$ particles. The enormous advances in detector technology over this last decade are best illustrated by constrasting the view of a $J / \psi$ event in the MARK I detector with the enormously more detailed "picture" from the UAl detector at CERN of an event with a $Z^{\circ}$, shown in Figure 10. The actual configuration of the immense 5000 ton UAl detector is shown in Figure 11 .

Fixed-target detectors typically have a more precise area of physics interest than the large collider detectors. The neutrino detectors are vast instrumented targets, usually incorporating magnetic analysis of produced muons and calorimetric measurements of produced hadrons. The target of a Fermilab neutrino detector, shown in Figure 12 , has an instrumented mass of 690 tons, consisting of $20-\mathrm{cm}$ iron slabs interleaved with drift chambers, followed by 420 tons of momentum analyzing toroidal magnets. Such large masses are required to achieve a reasonable interaction rate from the weakly interacting neutrinos. In fixed-target experiments, the kinematics of high-energy relativistic collisions results in most of the final-state particles from an interaction being thrown forward into a relatively narrow cone. Consequently, fixed-target experiments generally appear as a linear sequence of detector elements downstream from the target, as Figure 12 clearly illustrates.

The larger detectors constitute major facilities, with a 1 ifetime of usage tvpically more than 10 years. They cost in the range of 10 to 50 million dollars and compete for resources with even the large parent accelerators. Unlike the early pioneering experiments of 

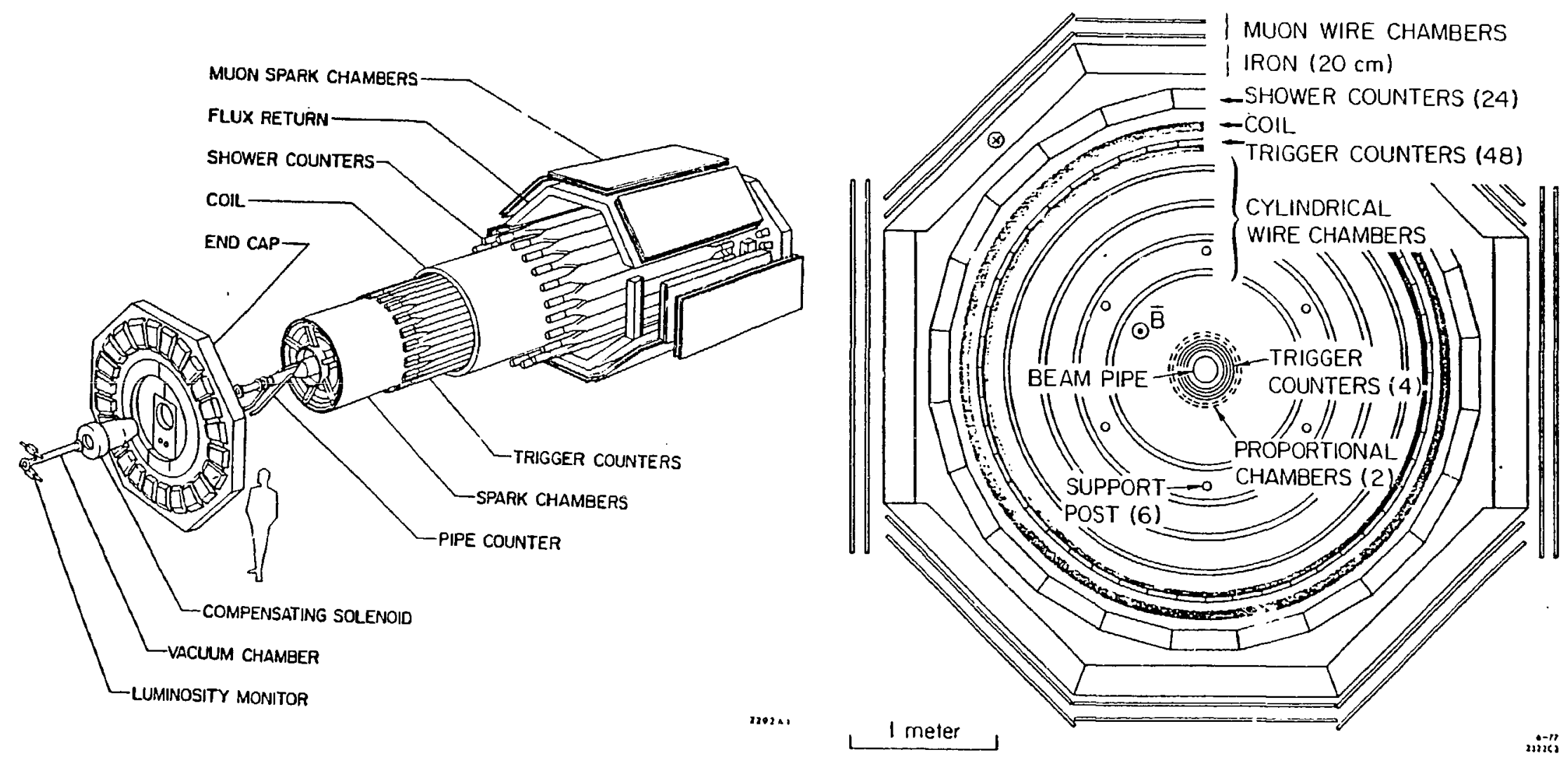

Fifice 8. (a) Mark l detector; (b) cross section. 


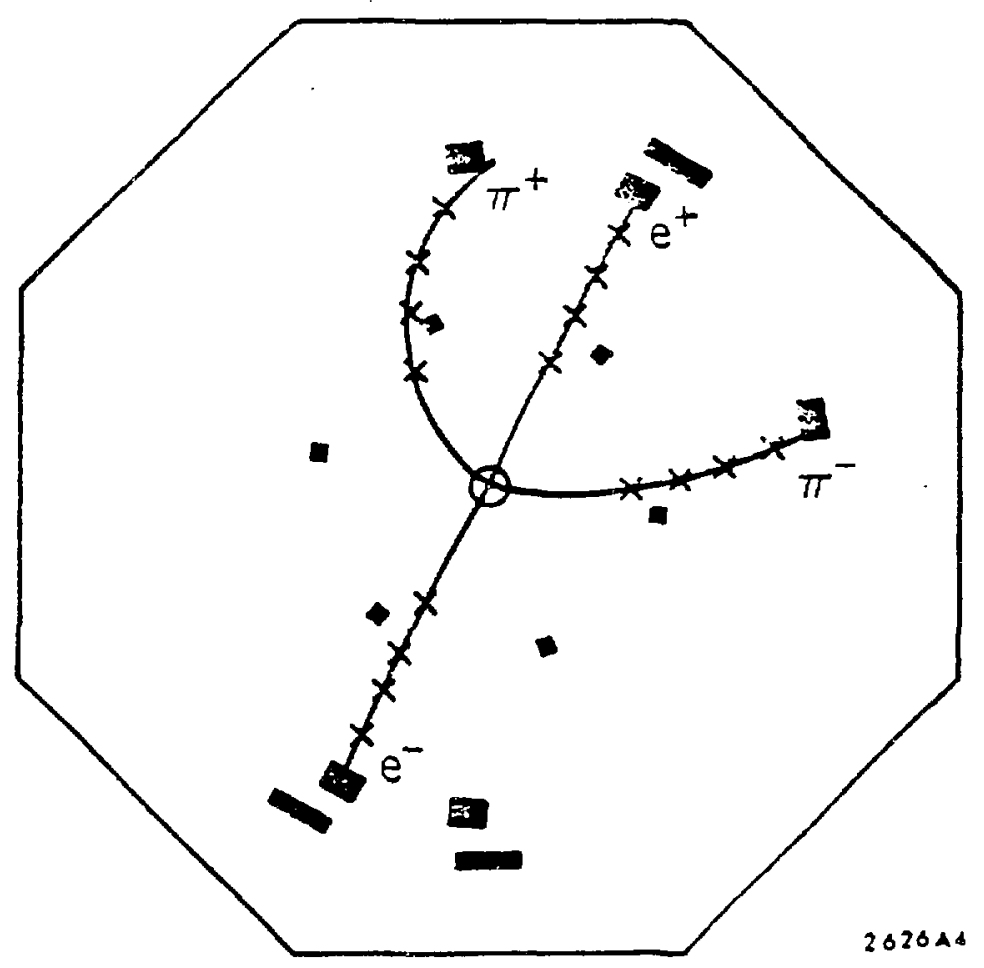

Figure 9. Reconstruction of $\mathrm{J} / \psi$ event.

particle fhysics, a modern experiment may well require the simultaneous collaboration of several hundred physicists from 20 or more institutions. These major facilities require resources comparable with those used in the construction of the parent accelerator.

A detector system should be able to measure, as completely as possible, all the characteristics of the produced particles within an event. This implies that the detector should function over the largest possible angular range, measure with the best attainable precision, be provided with instrumentation to identify particie characteristics, and simultaneously provide a wide range of cross checks to protect against measurement artifacts. Additionally, for use with hadron colliders, detectors must be able to extract interesting classes of physics events from backgrounds of events perhaps a hundred million times more frequent. These challenges must be met while simultaneously keeping the combined costs of construction, operation, and data-reduction within reasonable bounds.

The basic physics underlying detector operation can be sumarized as follows:

- Charged particles lose energy by ionization processes and leave a "track" or trail of ionized atoms and electrons as they pass th:ough gasses, liquids, or solids. A wide range of technigies serve to measure the position and magnitude of these ionization trails. The magnitude of this energy loss per unit length is a measure of particle velocities. 
Evevi

ㄱำ. : $25:$.
21

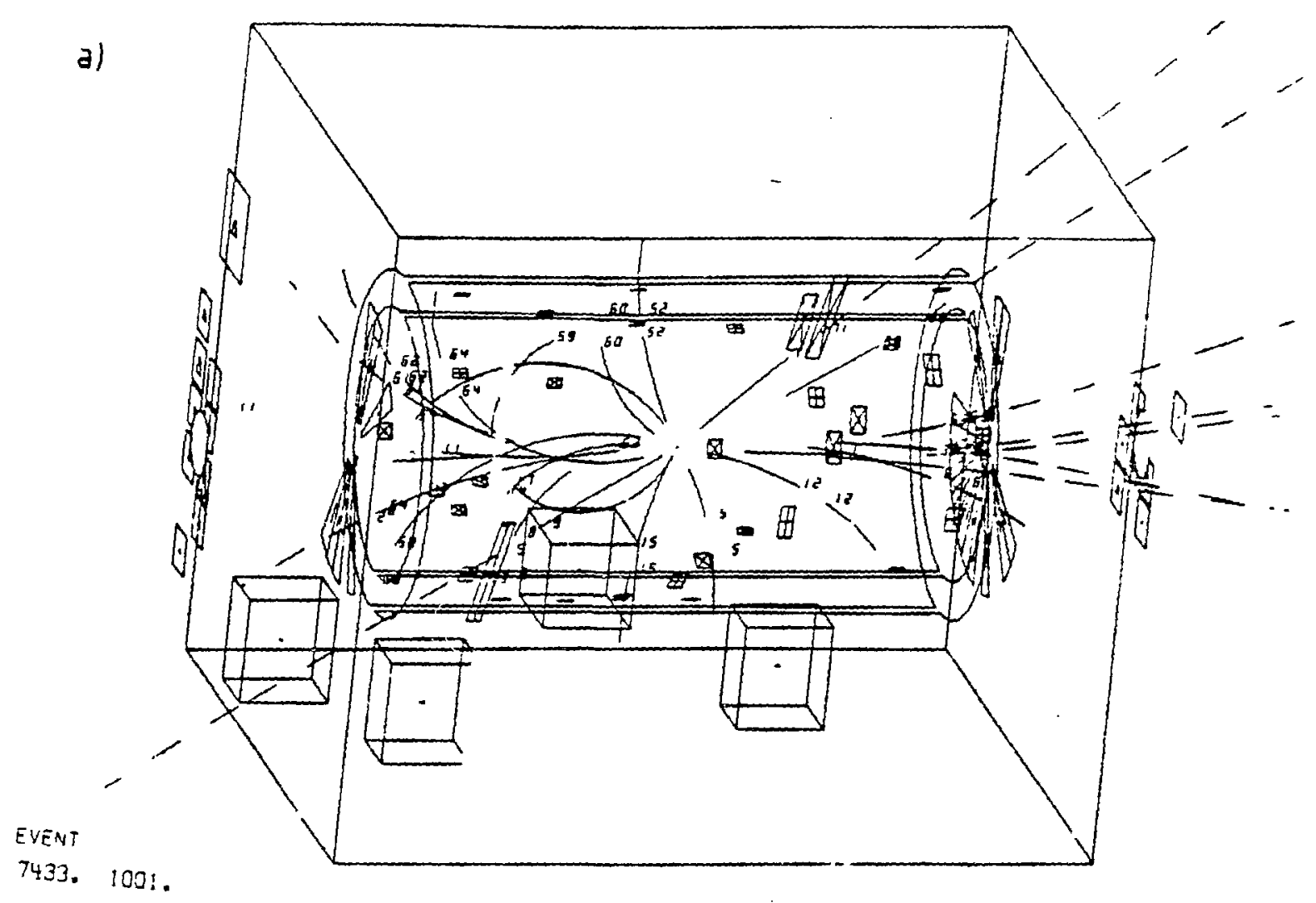

b)

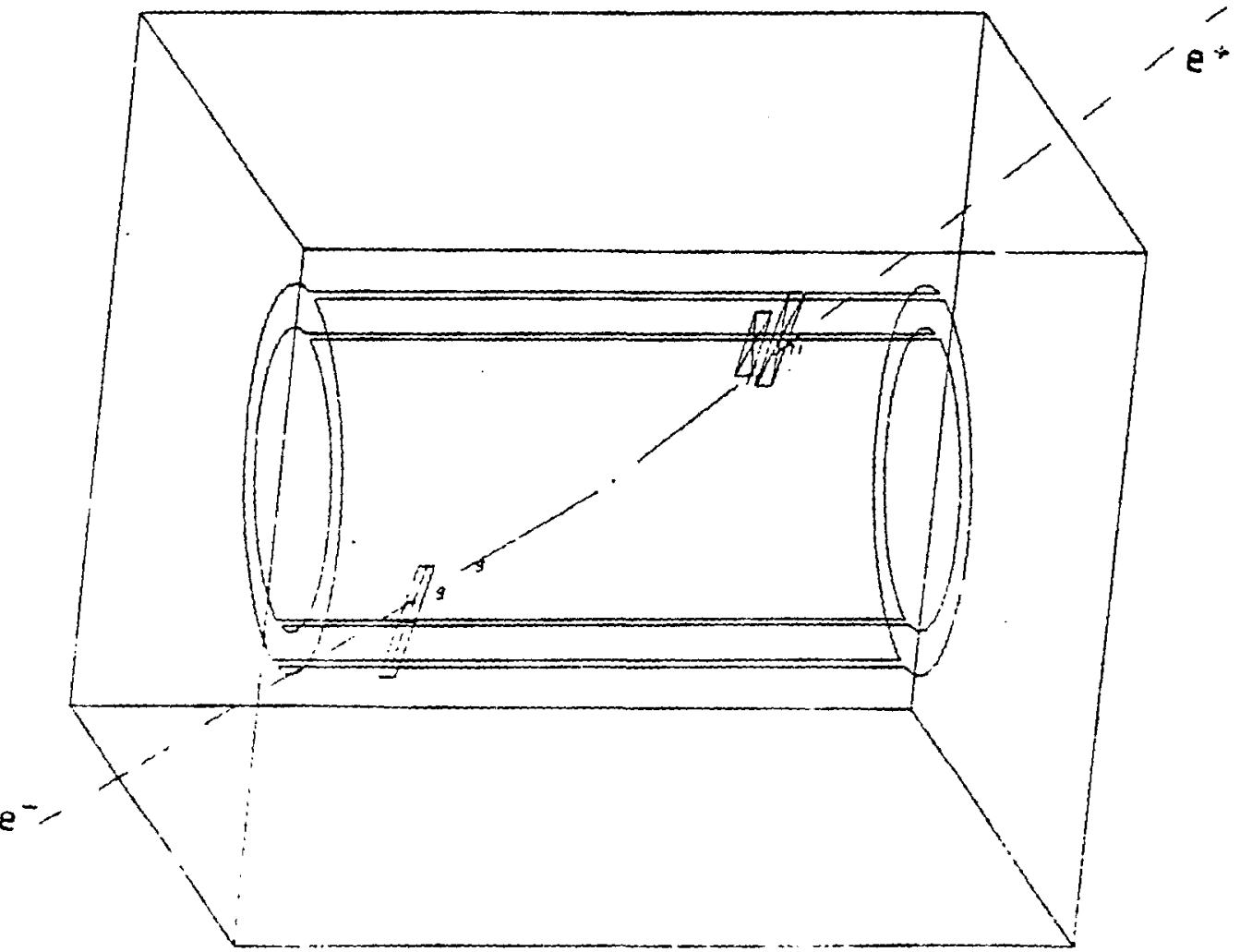

Figure 10. CERN UAl detector:

$z^{0}$ event. 


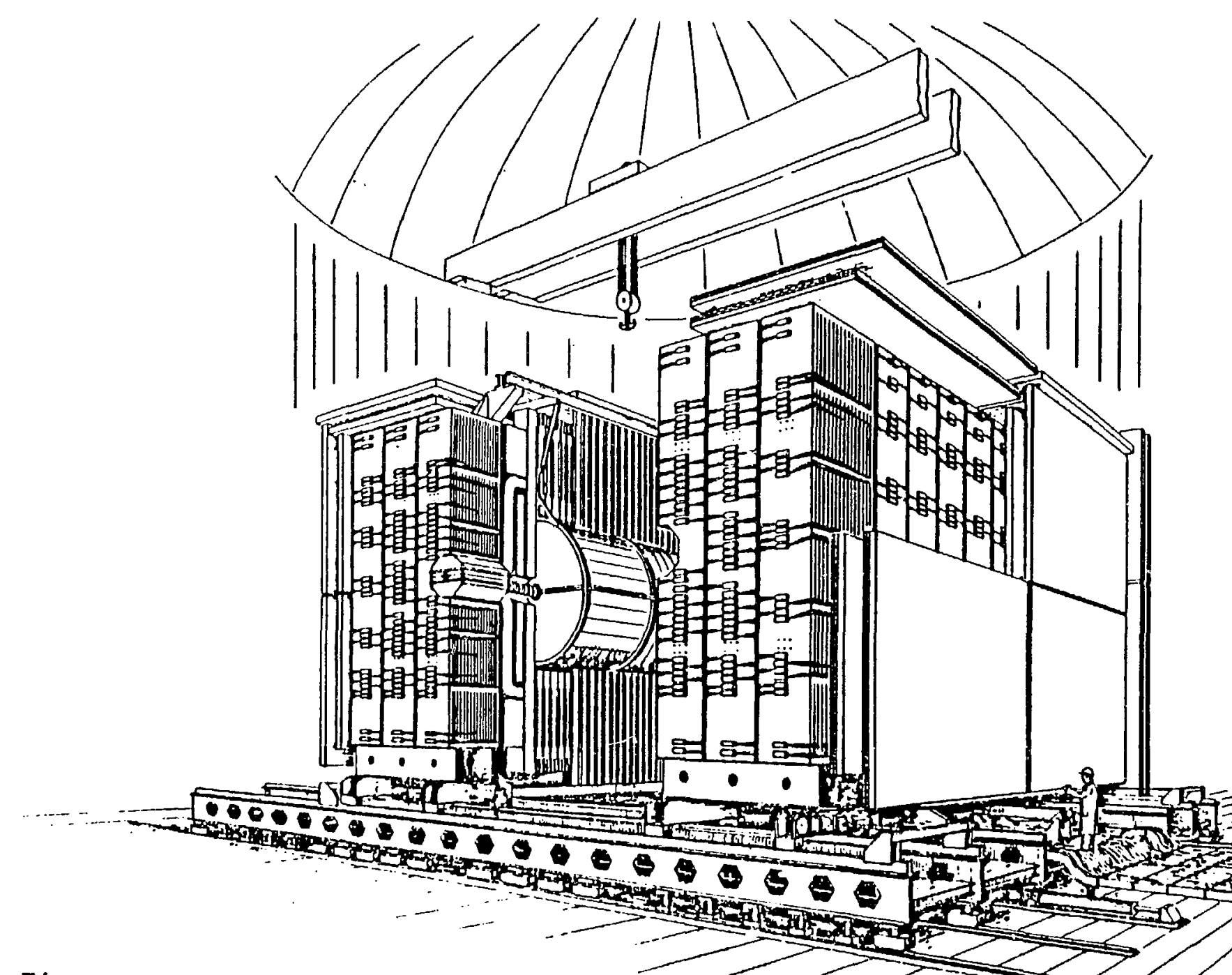

Figure 11. UAl detector configuration. 

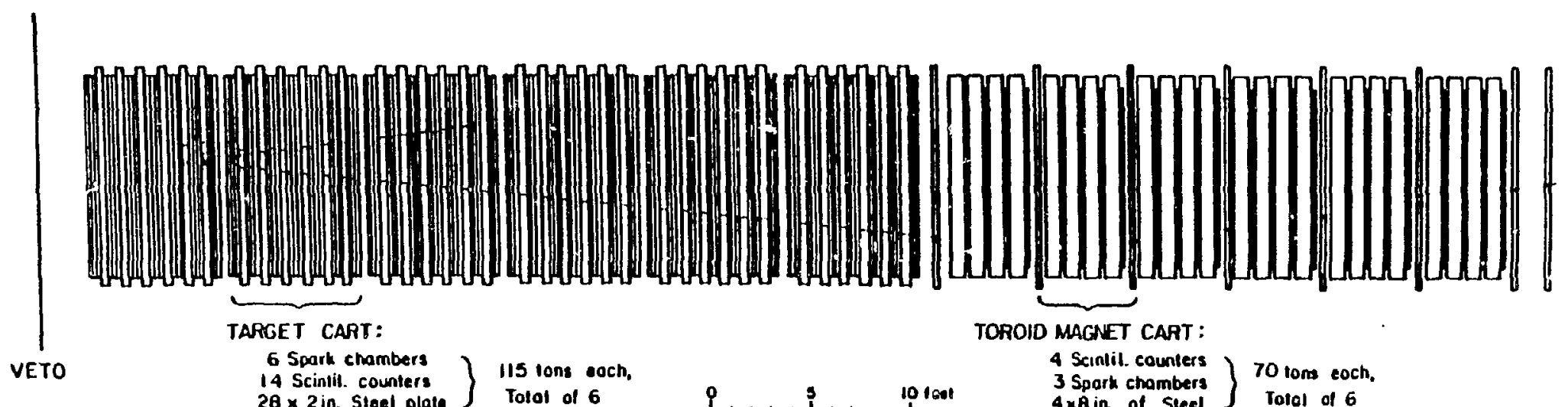

TOROID MAGNET CART :

$\left.\begin{array}{l}14 \text { Scintil. counters } \\ 20 \times 2 \text { in. Stoel plote }\end{array}\right\} \begin{aligned} & \text { Totol of } 6 \\ & \text {. }\end{aligned}$

$\left.\begin{array}{l}4 \text { Scinlil. Counters } \\ 3 \text { Spork chombers } \\ 4 \times 8 \text { in. of Sieel }\end{array}\right\} \begin{gathered}70 \text { tom eoch. } \\ \text { Tolol of } 6\end{gathered}$

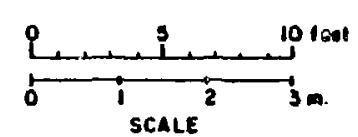

Figure 12. $\frac{E-6 ! 6 \text { NEUTRINO DETECTOR }}{690 \text { TON TARGET }}$
420 TON TOROID MAGNET

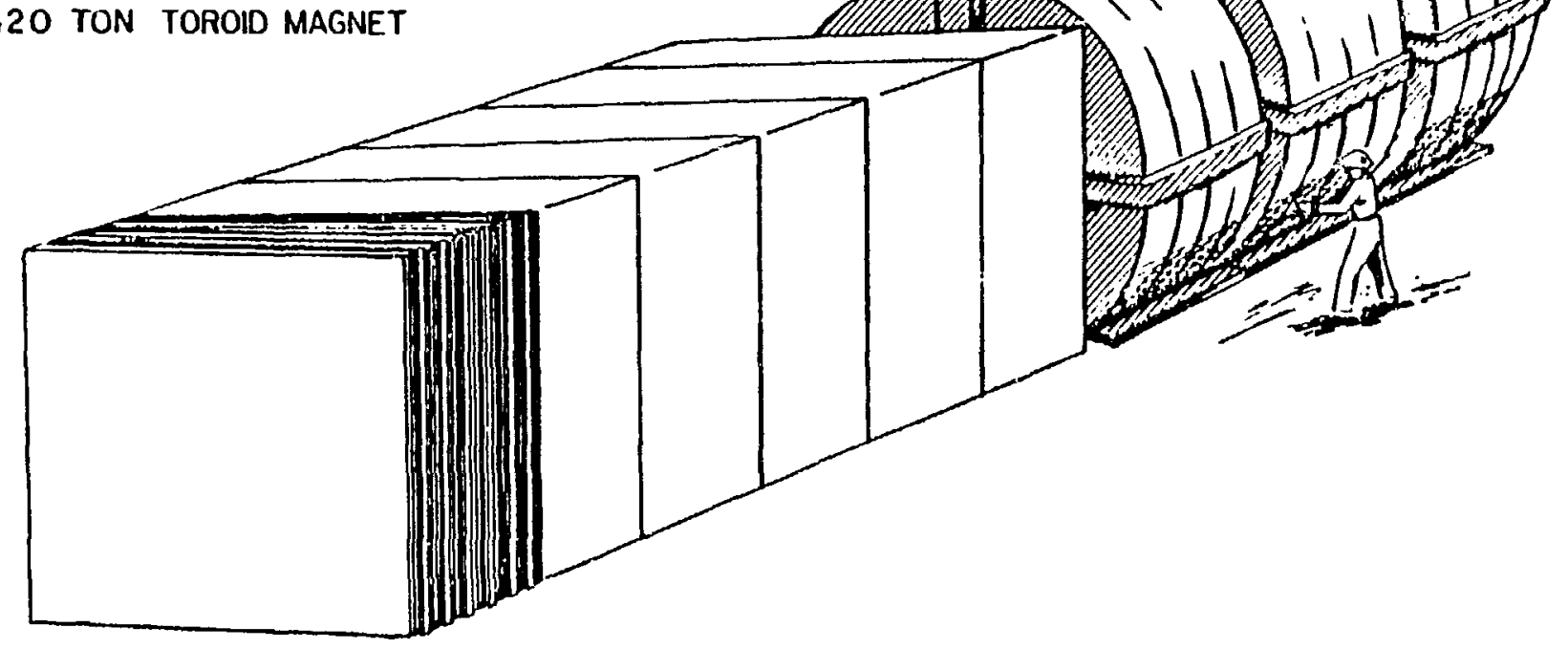


- Velocities of particles may be determined from the time interval required to pass between two points. In general this technique differentiates velocities only for relatively small particle energies.

- In the presence of a magnetic field, charged particles are deflected into curved orbits. Measurements of this curvature permits the momenta of these tracks to be determined. The particle's energy can be calculated from its momentum if the mass is known.

- Characteristic, but weak, radiation is coherently emitted by particles passing through material--Cerenkov radiation; or by particles as they cross an interface between different materials--transition radiation; or as they pass through magnetic fields--synchrotron radiation. The intensity and characteristics of these radiations can serve as the basis of a velocity measurement.

- When electrons or photons pass through matter they produce characteristic electromagnetic cascades of secondary radiation which in turn leave an intense core of ionized atoms. The energy of the original electron or photon is ultimately completely converted into ionization by these processes. Measurement of this converted energy in a sufficiently thick block of material determines the total incident electromagnetic energy and constitutes a calorimetric shower-energy measurement. A detector constructed to make use of this property is an eiectromagnetic calorimeter.

- Hadrons, such as protons and mesons, interact strongly as they pass through matter, producing secondary hadrons. This again results in the production of intense cores of ionized atoms. The total energy of the incident particles can be measured from the total energy deposited in the form of ionization. Hadronic cascades can be differentiated from the electromagnetic cascades that develop in much thinner layers of material. The technique of measurement is known as hadron calorimetry. Typically a hadron calorimeter might require a thickness of three to four feet of instrumented steel with a total weight of hundreds of tons.

- Energetic muons are uniquely characterized by the property that, although charged, they penetrate very large thicknesses of material and emerge with a relatively small change of energy at the outside of a detector.

Modern detectors typically make use of many or all of the above properties to characterize detected events. The characteristics of these detectors have many features in common and share very similar design architectures. The detectors for collider experiments are based on a series of concentric shells or layers, one behind the other, each of which is devoted to some particular aspect or aspects of the detection process. The initial detector layers are used to characterize the charged-particle component and are designed to be "nondestructive"-i.e, to contain very 1 ittle material so that charged particles will not interact or degrade in energy and thus will maintain their 
identity while traversing the layers. The outer detector layers deliberately use large amounts of material in order to materialize the neutral particles and to convert the energy carried by the particles into detectable ionization; such a device is known as a calorimeter.

The outer layer of the calorimeter, or an additional detection layer, is frequently used to detect muons. As mentioned earlier, energetic muons are usually the on $1 y$ particles that can reach this outermost layer.

Inevitably, as the layered levels of detection systems are built up, a detector will become very large and correspondingly complex and expensive: A prime objective of detector development is, therefore, to keep detection systems as compact as possible and to combine detection roles whenever possible.

Additional demands are imposed on detector systems associated with hadron colliders by the high ambient radiation levels at the detector, and by the fact that events of interest may be separated only by very short times from uninteresting hackground events.

The elements or layers constituting a typical modern detector can be seen in the schematic of the CDF detector at the Tevatron collider facility at Fermilab (Figures $13 a$ and $13 b$ ).

Detectors for the new colliders face many new challenges. For example, experiments at the Stanfecd Linear Collider (SLC) with its micron-size beams would benefit from improved spatial resolution if the existing 50 to 100-micron resolution of drift chambers could be improved to a level of about 5 microns. Micro-vertex detectors that can identify the secondary vertex when bottom or charm quarks are produced are being developed. Another important area for $R$ and $D$ is in the use of charge-manipulation structures of silicon or gallium arsenide for particle detection. Silicon strip detectors are being used successfully in particle physics experiments and have demonstrated 5-micron spatial resolution. Two-dimensional ChargeCoupled Devices (CCD) are being developed for television cameras and other optical imaging applications by industry; there is a clear benefit here in a collaborative approach both between laboratories and between laboratories and the micro-electronic industry.

To be effective and productive, the U.S. High Energy Physics Program requires a diversity of particle acccelerator and colliding beam facilities with adequate detector facilities. In the study of fundamental particles, valuable information comes also from sources other than accelerators. The detection of cosmic rays, measurements at nuclear reactors, the search for rare decays in the shielded environment of deep tunnels and mines, and the precise study of atomic transitions have all contributed to high energy physics. The most crucial and fundamental questions, however, can be answered only by probing deeper into the nucleon with increasingly energetic particle collisions. 


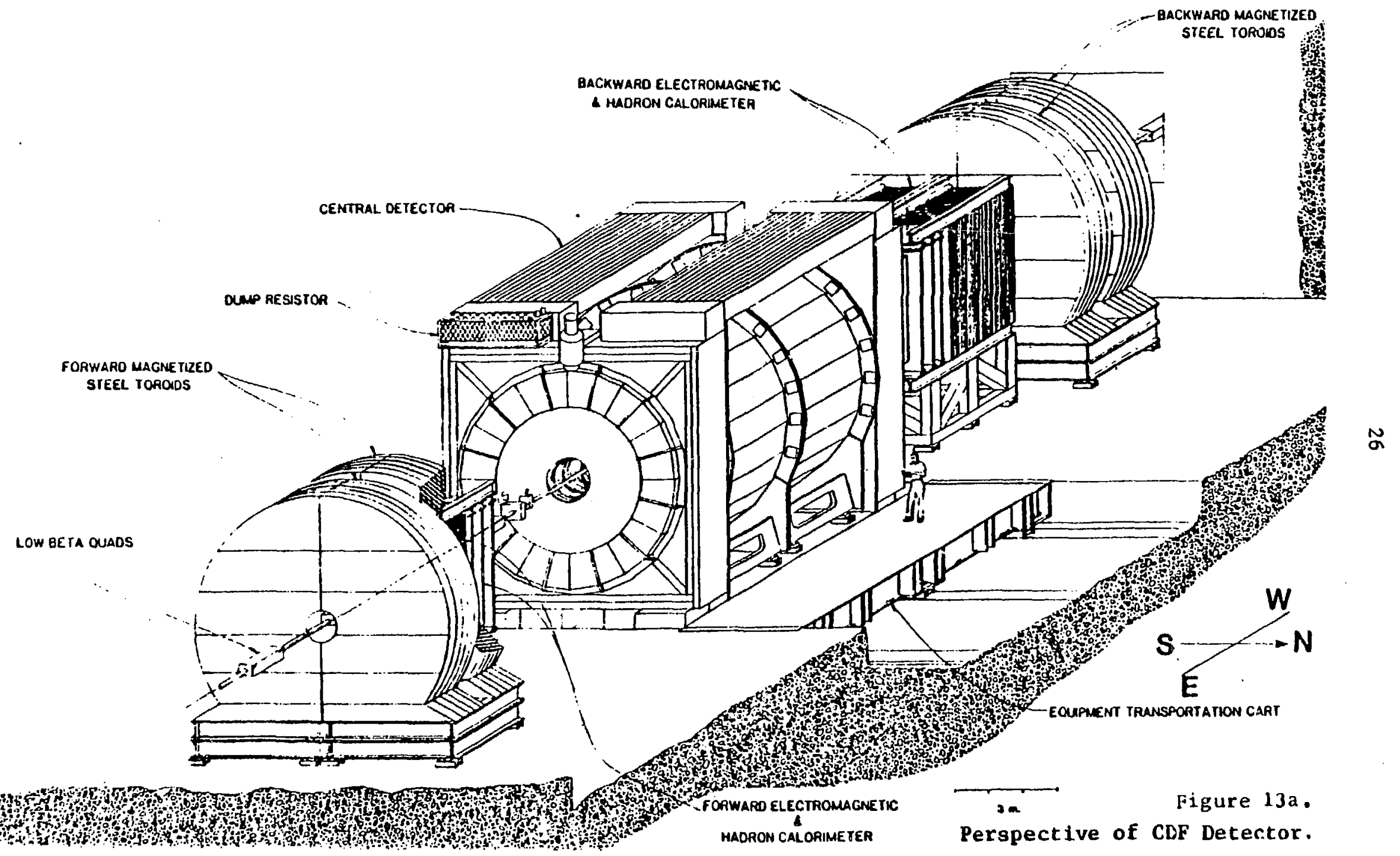




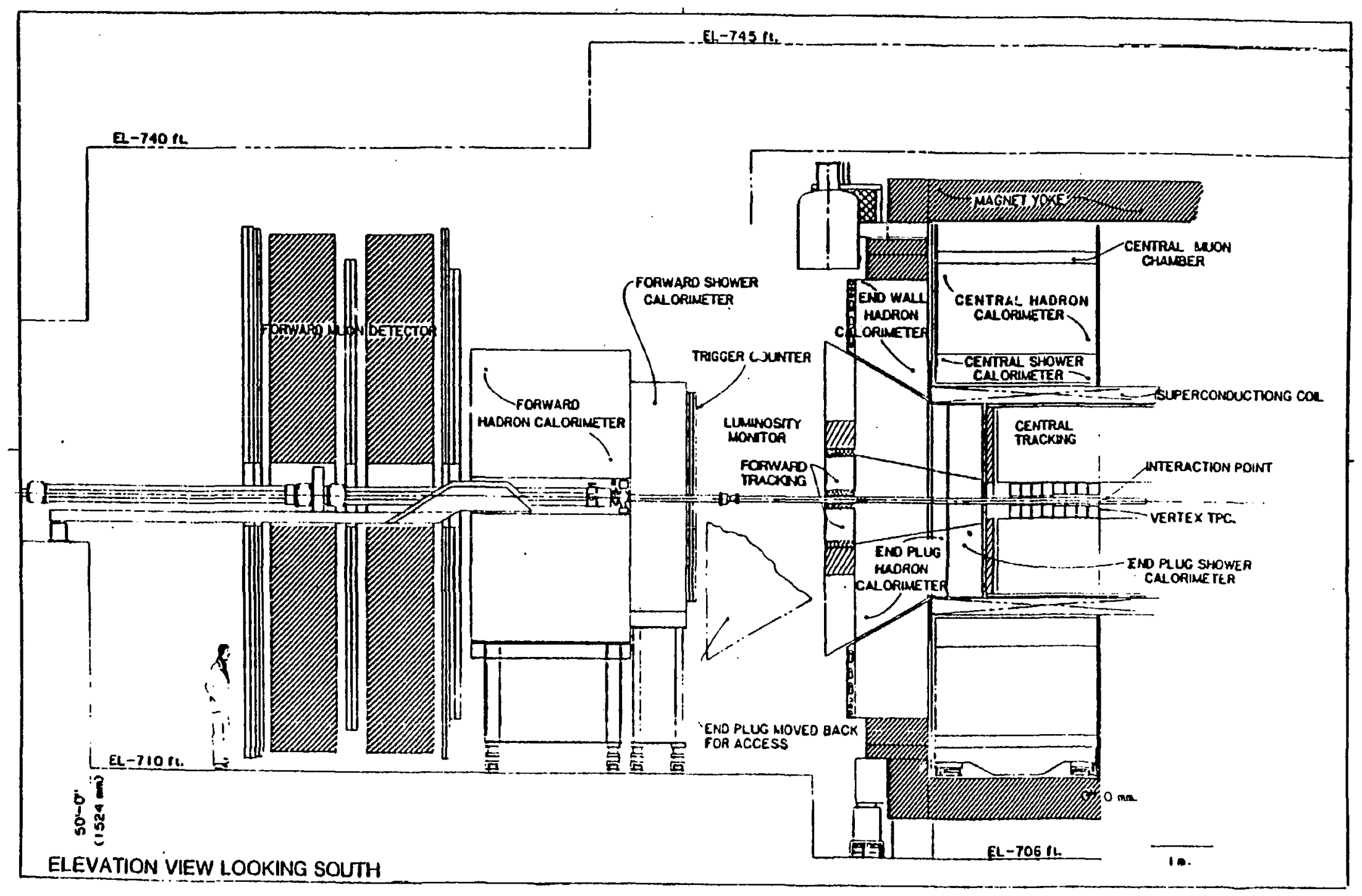

Figure 13b. Cross Section of CDF Decector. 


\section{A. Overview}

The U.S. program through $: 995$ will clearly have to depend on the major faciliti ss now operating and under construction. Assurance of a forefront U.S. program in this period will require that the already operating facilizies (the AGS at Brookhaven, the Energy Saver at Fermilab, the linac and the PEP and SPEAR colliders at SLAC, and the colliding beam device CESR at Cornel1) be thoroughly utilized and upgraded to the extent required by physics developments, and that the Fermilab Tevatron I and Tevatron II and the SLAC SLC facilities under construction be completed expeditiously and utilized effectively. The Fermilab and SLAC facilities are expected to carry the major burden of serving U.S. high energy physicists during this period.

These facilities coupled with the use of unique foreign facilities will permit a comprehensive program of research in the mass range below a few hundred $\mathrm{GeV}$. The existing ones will permit detailed studies of weak interactions, study of charm and bottom quark spectroscopy, and tests of QED and QCD. The new ones coming on line between now and 1987 at Fermilab and SLAC will open the regime of the $Z^{\circ}$ and $W$ and the electroweak interaction, top quark states, and any unexpected discoveries in the new energy regions.

The program will also provide opportunities for U.S. scientists to work at foreign facilities with unique capabilities not available here. The program will in addition include diverse nonaccelerator experiments aimed at exploring very low energy phenomena, which reveal critical features of the grand unified theories, as well as cosmic-ray physics and astrophysical processes.

As discussed in Section IV, there is an essential need for a major new facility to come into operation in the mid-1990s, the proposed Superconducting Super Collider (SSC), a device which would collide head-on two beams of protons, each of energy $20 \mathrm{TeV}$. An extensive program of preconstruction research and development will be carried out for several years beginning in FY 1984 to establish a cost-optimized design for this facility. This $R$ and $D$ will provide the data for a conceptual design, will integrate technical requirements with cost-effective fabrication methods, and will provide realistic cost and schedule estimates.

If the SSC is approved for a start at the end of the $1980 \mathrm{~s}$, then the 13te 1980s and early 1990s will be a period of extensive preparation for experiments at this facility. Probably some existing capabilities would have to be redirected so that the needed fiscal and manpower resources are available to develop the new detectors required for effective utilization of the opportunities provided by the SSC (see Figure 14).

In this period, the program would include a strong component of advanced technology $R$ and $D$ on future accelerator and colliding beam systems with even higher energies or with different particle capabilities. Of major interest, assuming success of the Stanford Linear Collider (under construction), would be the investigation of energy 


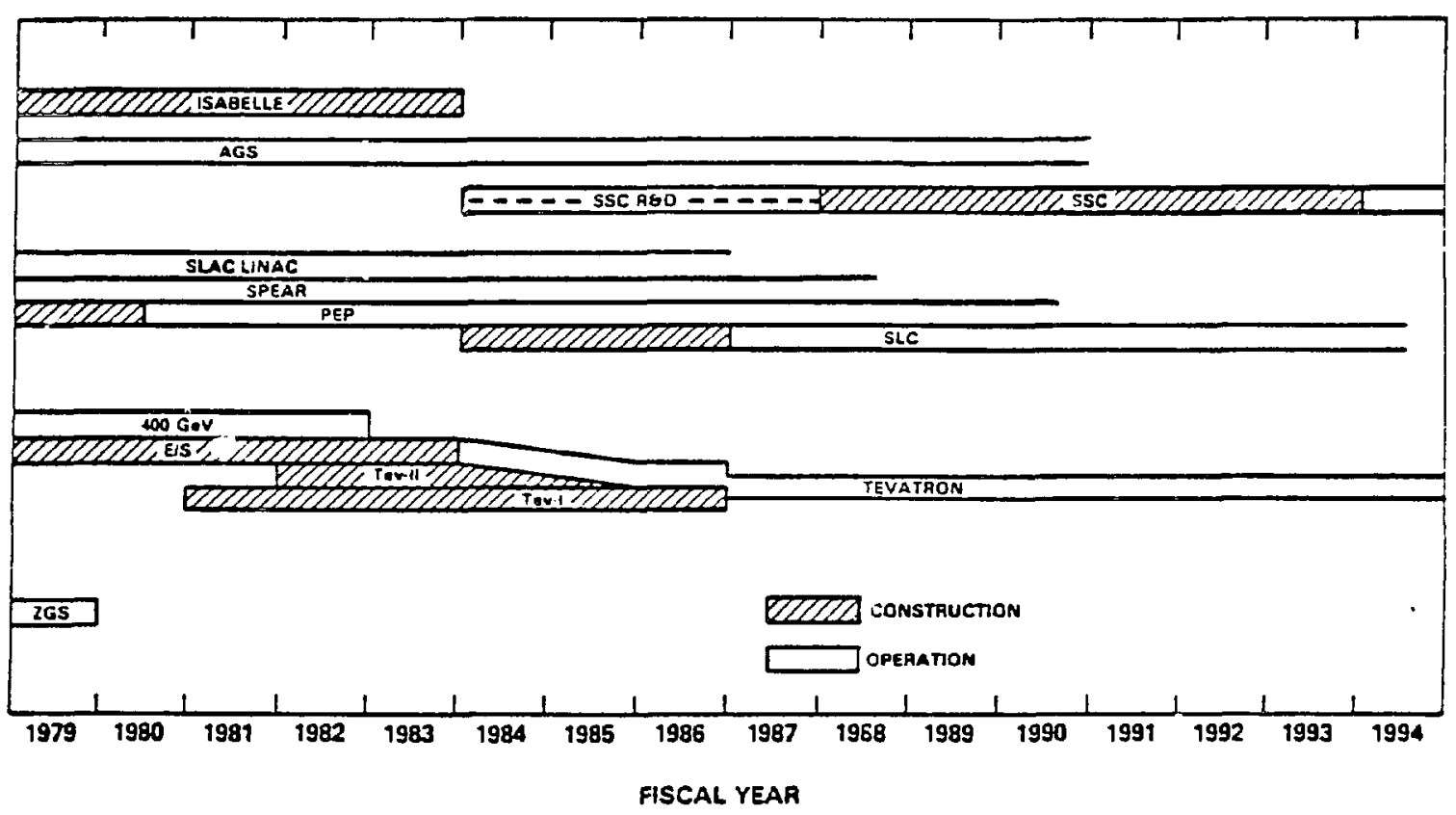

Figure 14. The time line indicates a possible plan for the future utilization of major DOE-funded accelerator facilities.

efficient acceleration structures and power systems for a large electron positron linear collider in the $\mathrm{TeV}$ energy range. Advantage would be taken of novel acceleration or detection schemes as the opportunities arise.

\section{B. Details of the Program}

The U.S. High Energy Physics Program at present depends primari)y on the accelerator facilities at the three DOE-supported national accelerator centers: Brookhaven National Laboratory (BNL), Fermi National Accelerator Lahoratory (Fermilab), and Stanford Linear Accelerator Center (SLAC). In addition, there is the CESR colliding beam facility at Cornell University supported by the National Science Foundation. Each has unique features complementary to those of the others, and together they provide the capabilities essential for a forefront U.S. program in the late 1980 s and early 1990s. The DOE three-center approach for high energy physics facilities has proven very effective in the past and, though faced with serious challenges from abroad, the U.S. continues to be recognized as a world leader in the field.

This section describes U.S. accelerator facilities (see Table IV); those in other countries are described in Section III. For comparison, Figure 15 shows the energy and luminosity at world-class high energy colliding beam facilities in operation, under construction, or being designed, and Tables $V$ and VI give more details of the U.S. high energy physics research centers and the major foreign ones. The U.S. High Energy Physics Program from 1985 to 1995, with emphas is on the major facilities existing or under construction, will be a vital program for the next decade. The planned SSC, if approved, 
is anticipated to come on line about 1995. The transition to the SSC is discussed in Section IV.

Table IV wajor L.S. Yigh Energy ?hysics Facj.lities

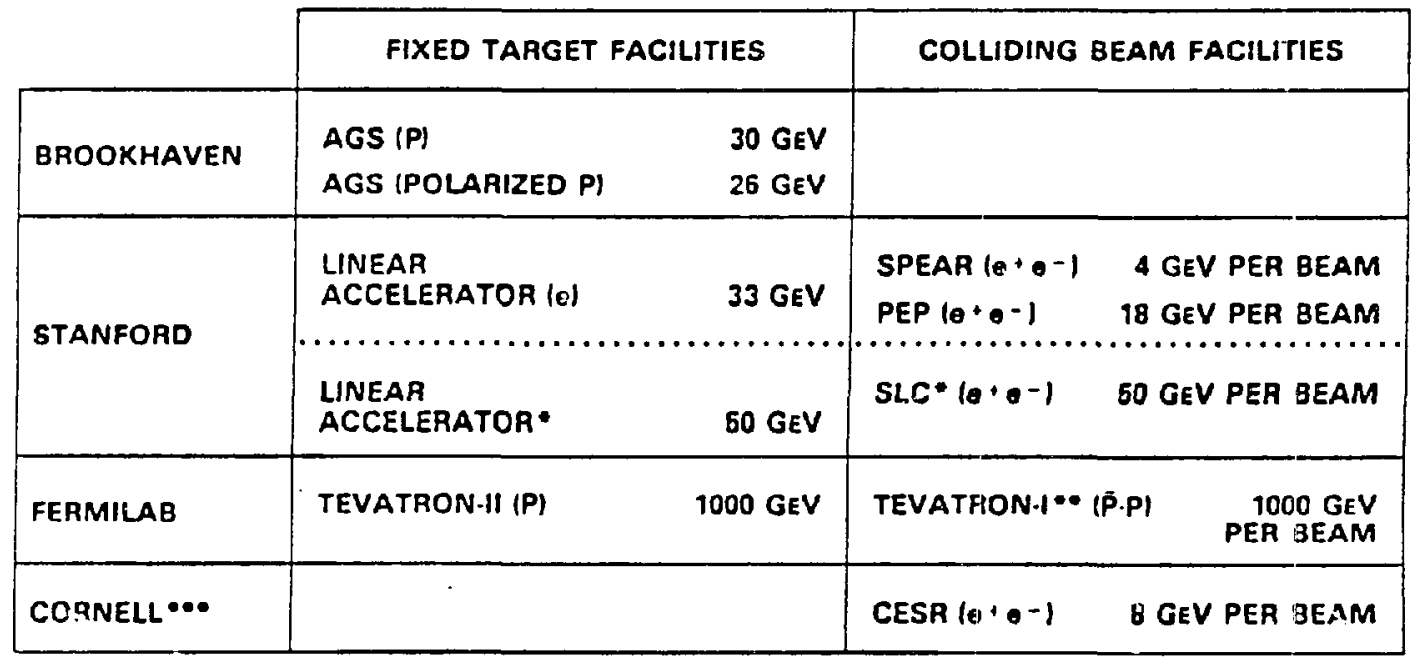

- linAC UPGRAde \& SLC CONSTRUCTION: INITIAL OPERATION FALL 1986.

- TEVATRON-I CONSTRUCTION: INITIAL OPERATION FALL 1986.

* NSF SUPPORTED.

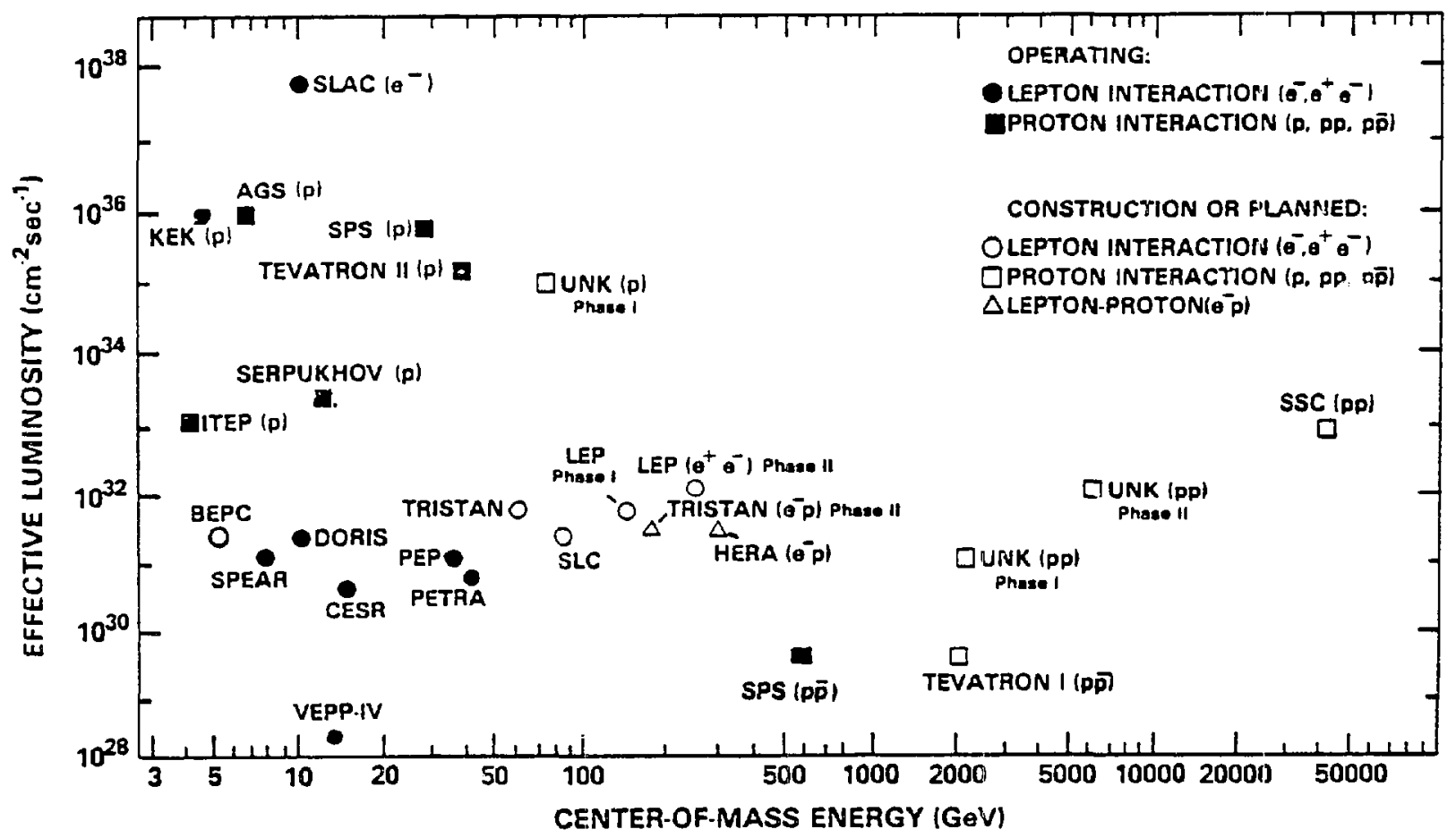

Figure 15. World accelerator and colliding-beam facilities. The major parameters, center-of-mass energy and luminosity. for colliding beam facilities in operation, under construction, or planned. The Superconducting Super Collider (SSC) would reach a center-of-mass energy of $40 \mathrm{TeV}$ at a luminosicy of $10^{33} \mathrm{~cm}^{-2} \mathrm{sec}^{-1}$, far surpassing the other facilities. 
Table V U.S. High lanergy Physics Research Centers

\begin{tabular}{|c|c|c|c|c|c|c|c|}
\hline $\begin{array}{l}\text { LABORATORY } \\
\text { (CITY. STATE) }\end{array}$ & TYPE OF MACHINE & PARTICLES & $\begin{array}{l}\text { MAXIMUM } \\
\text { ENERGY |GEV| }\end{array}$ & $\begin{array}{l}\text { CENTER OF } \\
\text { MASS ENERGY } \\
\text { (GEV) }\end{array}$ & $\begin{array}{l}\text { EFFECTIVE } \\
\text { LUMINOSITY } \\
\text { (cm } 2 \text { soc- } 1 \text { ) }\end{array}$ & $\begin{array}{l}\text { FIRST OPERATION } \\
\text { IN THIS MODE }\end{array}$ & STATUS \\
\hline $\begin{array}{l}\text { BROOKHAVEN NATIONAL } \\
\text { LABORATORY } \\
\text { (UPTON, LI, NYI }\end{array}$ & SYNCHROTRON (AGS) & $\begin{array}{l}\text { P } \\
\text { P (polarized) }\end{array}$ & $\begin{array}{l}30 \\
26\end{array}$ & $\begin{array}{l}7.5 \\
7.0\end{array}$ & $\begin{array}{c}1.3 \times 10^{38} \\
10^{33}\end{array}$ & $\begin{array}{l}\text { JULY, } 1960 \\
\text { JULY, } 1984\end{array}$ & $\begin{array}{l}\text { OPERATING } \\
\text { OPERATING }\end{array}$ \\
\hline $\begin{array}{l}\text { CORNELL U. } \\
\text { (ITHACA, NY) }\end{array}$ & STORAGE RING (CESA) & $\theta^{\prime} \theta$ & $8 \times 8$ & 16 & $7 \times 10^{30}$ & JUNE, 1979 & OPERATING \\
\hline $\begin{array}{l}\text { FERMILAB } \\
\text { (BATAVIA, ILI }\end{array}$ & $\begin{array}{l}\text { SYNCHAOTRON (TEV-II) } \\
\text { STORAGE RING (TEV.I) }\end{array}$ & $\begin{array}{l}\mathbf{p} \\
\mathbf{p p}\end{array}$ & $\begin{array}{l}1.000^{\circ} \\
1.000 \times 1.000\end{array}$ & $\begin{array}{r}43^{*} \\
2,000^{*}\end{array}$ & $\begin{array}{l}2 \times 10^{35} \\
6 \times 10^{25}\end{array}$ & $\begin{array}{c}(1983-1986) \\
(1986)\end{array}$ & $\begin{array}{c}\text { OPERATING } \\
\text { UNDER CONSTR. }\end{array}$ \\
\hline $\begin{array}{l}\text { SLAC } \\
\text { (PALO ALTO, CA) }\end{array}$ & $\begin{array}{l}\text { LINAC ISLED.II } \\
\text { LINAC IUPGRADE) } \\
\text { STORAGE RING ISPEARI } \\
\text { STORAGE RING (PEPI } \\
\text { LINEAR COLLIDER (SLC) }\end{array}$ & $\begin{array}{l}\theta^{\circ} \cdot \theta^{\circ} \\
\theta^{\circ}, \theta^{+} \\
\theta^{\circ} \theta^{-} \\
\theta^{\circ} \theta^{-} \\
\theta^{\circ} \theta^{-}\end{array}$ & $\begin{array}{c}33 \\
50^{\circ} \\
4.2 \times 4.2 \\
18 \times 18 \\
60 \times 50^{\circ}\end{array}$ & $\begin{array}{c}7.9 \\
9.7 \\
8.4 \\
36 \\
100\end{array}$ & $\begin{array}{l}10^{30} \\
7 \times 10^{37} \\
10^{31} \\
5 \times 10^{30} \\
6 \times 10^{30}\end{array}$ & $\begin{array}{c}1976 \\
\text { (1986) } \\
\text { APRIL. } 1972 \\
\text { MAY, 1980 } \\
\text { [1986) }\end{array}$ & $\begin{array}{l}\text { OPERATING } \\
\text { UNDER CONSTR. } \\
\text { OPERATING } \\
\text { OPERATING } \\
\text { UNDER CONSTR. }\end{array}$ \\
\hline UNSPECIFIED & $\begin{array}{c}\text { SUPERCONDUCTING SUPER } \\
\text { COLLIDER (SSC) }\end{array}$ & pp & $20.000 \times 20.000^{*}$ & $40,000^{\circ}$ & $10^{33} \cdot$ & $|\sim 1994|$ & PROPOSED \\
\hline
\end{tabular}

the numbea given is a design goal, not a measured value.

DECEMBER 12, 1984 
Table VI Major Foreign lligh thergy rliysics llesearch Centers

\begin{tabular}{|c|c|c|c|c|c|c|c|c|}
\hline LOCATION & $\begin{array}{l}\text { LABORATOAY } \\
\text { (CITY) }\end{array}$ & TYPE DF MACHINE & PAATICLES & $\begin{array}{l}\text { MAXIMUM } \\
\text { ENEAGY (GEV) }\end{array}$ & $\begin{array}{l}\text { CENTER OF } \\
\text { MASS ENEAGY } \\
\text { |GEVI }\end{array}$ & $\begin{array}{l}\text { EFFECTIVE } \\
\text { LUMINOSITY } \\
\left\{\mathrm{Gm}-\mathbf{2}_{\text {GOC }}^{-1} \mid\right.\end{array}$ & $\begin{array}{l}\text { FIFST OPEAATION } \\
\text { IN THIS MODE }\end{array}$ & STATUS \\
\hline \multirow[t]{2}{*}{ WESTERN EUROPE } & $\begin{array}{l}\text { CERN } \\
\text { IGENEVA, } \\
\text { SWITZERLANDI }\end{array}$ & $\begin{array}{c}\text { SYNCHROTRON AND } \\
\text { STORAGE RING (SPS) } \\
\text { STORAGE RING (LEP) } \\
\text { PHASE I } \\
\text { PHASE II }\end{array}$ & $\begin{array}{c}\text { p } \\
\text { pp } \\
0 \cdot \bullet-\end{array}$ & $\begin{array}{l}450 \\
270 \times 270 \\
60 \times 60^{\circ} \\
130 \times 130^{\circ}\end{array}$ & $\begin{array}{r}29 \\
640 \\
120^{\circ} \\
260^{\circ}\end{array}$ & $\begin{array}{l}6 \times 10^{36} \\
6 \times 10^{21} \\
4 \times 10^{31} \\
\sim 10^{32}\end{array}$ & $\begin{array}{l}\text { MAY. } 1976 \\
\text { JULY. } 1981 \\
\text { I1898) } \\
-\end{array}$ & $\begin{array}{l}\text { OPERATING } \\
\text { OPEAATINO } \\
\text { UNDER CONSTR. } \\
\text { (IN PLANNING) }\end{array}$ \\
\hline & $\begin{array}{l}\text { DESY } \\
\text { IHAMBURG. } \\
\text { GERMANY! }\end{array}$ & $\begin{array}{c}\text { DORIS } \| \\
\text { PETRA UPGRADE } \\
\text { STORAGE RINGS (HERAI }\end{array}$ & 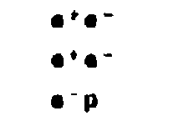 & $\begin{array}{l}6.6 \times 5.6 \\
23 \times 23 \\
30 \times 820^{\circ}\end{array}$ & $\begin{array}{l}11.2 \\
46 \\
314\end{array}$ & $\begin{aligned} & \times 10^{310} \\
\sim 6 & \times 10^{30} \\
4 & \times 10^{310}\end{aligned}$ & $\begin{array}{l}\text { JULY, } 1982 \\
\text { OCTOBER. 1993 } \\
\text { (1990) }\end{array}$ & $\begin{array}{l}\text { OPERATINO } \\
\text { OPERATING } \\
\text { UNDER CONSTR. }\end{array}$ \\
\hline JAPAN & $\begin{array}{l}\text { KEK } \\
\text { (TSUKUBA) }\end{array}$ & $\begin{array}{l}\text { SYNCHROTRON (KEK) } \\
\text { STORAGE RINGS (YRISTAN) } \\
\text { PHASE I } \\
\text { PHASE II }\end{array}$ & $\because p$ & $\begin{array}{l}12 \\
30 \times 30= \\
25 \times 300^{\circ}\end{array}$ & $\begin{array}{c}6 \\
60 * \\
173 \cdot\end{array}$ & $\begin{array}{l}10^{34} \\
\times 10^{31} \\
6 \times 10^{311}\end{array}$ & $\begin{array}{c}\text { MARCH. } 1976 \\
\text { I1898 } \\
-\end{array}$ & $\begin{array}{l}\text { OPERATING } \\
\text { UNDER CONSTR. } \\
\text { (IN PLANNING) }\end{array}$ \\
\hline PAC & $\begin{array}{l}\text { IHEP } \\
\text { (BEIJING| }\end{array}$ & $\begin{array}{l}\text { SYNCHAOTRON (BPS) } \\
\text { STORAGE RING (BEPC) }\end{array}$ & $\stackrel{p}{p \cdot-}$ & $\begin{array}{c}60^{\circ} \\
2.5 \times 2.5^{\circ}\end{array}$ & $\frac{10^{\circ}}{5^{\circ}}$ & $\begin{array}{l}\sim 10 \times 0 \\
\sim 5 \times 10^{211}\end{array}$ & - & $\begin{array}{l}\text { (PSTPND. INDEF.) } \\
\text { UNDEA CONSTA. }\end{array}$ \\
\hline USSA & $\begin{array}{l}\text { IHEP } \\
\text { ISERPUKHOVI } \\
\\
\text { INSTITUTE OF } \\
\text { NUCL. PHYSICS } \\
\text { (NOVOSIBIRSKI } \\
\text { ITEP IMOSCOWI }\end{array}$ & $\begin{array}{c}\text { SYNCHAOTRON } \\
\text { SYNCHROTRON AND } \\
\text { STORAGE RING IUNK) } \\
\text { PHASE I } \\
\text { PHASE II } \\
\text { STORAGE RING IVEPP.IV) } \\
\text { STORAGE RING (VAPP.IV) } \\
\text { SYNCHROTRON (U.10) }\end{array}$ & $\begin{array}{c}p \\
p \\
p p \\
p p \\
p \cdot- \\
p p\end{array}$ & $\begin{array}{c}76 \\
3,000^{\circ} \\
400 \times 3,000^{\circ} \\
3,000 \times 3,000^{-} \\
7 \times 7 \cdot \\
25 \times 25 \\
10\end{array}$ & $\begin{array}{r}12 \\
75^{\circ} \\
2.190^{\circ} \\
6.600^{\circ} \\
14^{\circ} \\
60^{\circ} \\
4\end{array}$ & $\begin{array}{c}6 \times 10^{33} \\
\sim 10^{360} \\
\sim 10^{31} \\
10^{32} \\
3 \times 10^{20} \\
\text { IUNKNOWNI } \\
\text { IUNKNOWNI }\end{array}$ & $\begin{array}{l}1967 \\
\text { [1990. PROJECTED) } \\
\text { [1990. PRO.JECTED) } \\
- \\
1979 \\
\text { (UNKNOWN] } \\
\text { (OCTOBER, 1961) }\end{array}$ & $\begin{array}{l}\text { OPERATING } \\
\text { UNDER CONSTR. } \\
\text { UNDER CONSTR. } \\
\text { PLANNED } \\
\text { OPERATING } \\
\text { IUNKNOWNI } \\
\text { OPEAATING }\end{array}$ \\
\hline
\end{tabular}




\section{Brookhaven National Laboratory (BNL)}

The fixed-target experimental program at BNL is based on the AIternating Gradient Synchrotron (AGS), a $30-\mathrm{GeV}$ proton synchrotron that provides intense external beams of high energy protons and also secondary beams of pions and kaons and low energy neutrino beams. A schematic of the present BNL accelerator facility is given in Figure 16. Many major discoveries have been made at the AGS, such as the finding that the neutrino associated with the electron is different from that associated with the muon; the omega minus hyperon, which gave strong evidence for the quark hypothesi:; $C P$ violation and, indirectly, the violation of time-reversal invariance, which led to a Nobel Prize for J. Cronin and V. Fitch in 1980; the J/W particle, which led to a Nobel Prize for S.C.C. Ting and B. Richter in 1976; and the charmed baryon. Currently the physics program at the AUs focuses on studies of the weak interactions via neutrino interactions and rare $\mathrm{R}$-meson decays; the search for neutrino oscillations; hadronic spectroscopy, including the study of gluonium states; a program of spin physics using the polarized proton beam; OED, via measurements on muonic atoms with helium nuclei; intermediate energy nuclear physics studies on hypernuclei including hyperon-induced atomic $x$-ray emission; and a search for an exotic six-quark state, termed $H$.

The AGS is the only proton accelerator in the world operating for physics experiments in the energy range up to $30 \mathrm{GeV}$. In addition, it provides more than $10^{13}$ protons per pulse every 1.2 seconds for neutrino physics and, with a one-second flattop every 2.5 seconds, for electronic counter experiments.

Vigorous work is in progress to improve the AGS performance so that more sensitive experiments can be done in the very interesting areas of rare $k$ decays and neutrino oscillations. In addition, a more intense polarized proton source is being developed and will enhance this unique BNL capability. Soon it will be possible to accelerate heavy ions in the AGS with initially the availability of ions up to mass 32 and energies up to $15 \mathrm{GeV}$ per nucleon. A booster synchrotron

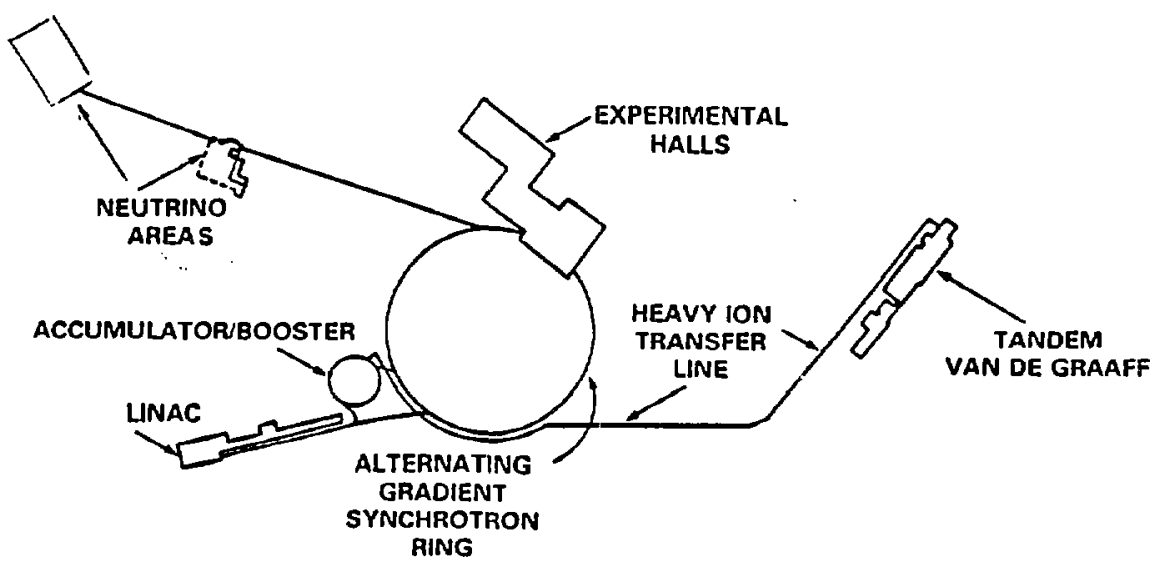

Figure 16. BNL high energy accelerator facilities. 
is being considered as an addition to the AGS in the late 1980 s to allow operation with significantly increased proton and polarized proton bean intensities, to permit sumultaneous operation of the Fast External Beam and the Slow External Beam as well as to extnd the mass range of accelerated heavy ions. In the future, it may be possible that these facilities could be used to inject heavy ions into a 100 on $100-\mathrm{GeV} /$ nucleon collider located in the now unused CBA tunne 1 . This proposed Relativistic Heavy Ion Collider (RHIC) could allow the study of the high baryon density phase transition and quark-gluon plasmas.

\section{Stanford Linear Accelerator Center (SLAC)}

The SLAC linear accelerator is the highest energy and highest intensity electron accelerator in the world. It currently provides beams of $33-\mathrm{GeV}$ electrons and $22-\mathrm{GeV}$ positrons and serves as injector for the SPEAR and PEP colliding beam facilities. Its energy is being upgraded to $50 \mathrm{GeV}$ so that it can serve as the central element in the Stanford Linear Collider (SLC). A schematic is given in Figure 17 .

a. SLAC Fixed-Target Program. Since its start in 1966 , the fixed-target experimental program at SLAC has contributed great $1 \mathrm{v}$ to the elucidation of the laws of physics. It gave rise to such pioneer-ring experiments as electron-nucleon inelastic scattering, which gave the first dynamic evidence for the quark and gluon composition of the nucleons, and the parity violation in polarized electron-deuteron scattering, which helped to establish the validity of the GlashowWeinberg-Salam model of the unified electroweak force. The program also included extensive photoproduction experiments and a spectrum of experiments with secondary hadron beams to study the hadron spectroscopy and weak interactions in $\mathrm{K}$-meson decays.

In recent years, the linear accelerator has been used mainly as an injector for the SPEAR and PEP storage rings, and the fixed-target program has been winding down. Recent experiments have included an axion search and a charmed particle lifetime measurement in the SLAC Hybrid Bubble Chamber facility. This has been completed, and no further bubble chamber experiments are planned. Another experiment, designed to study the scattering of electrons on nuclei and to eluci-

Figure 17. Stanford Linear Accelerator Center. High energy physics accelerator facilities.

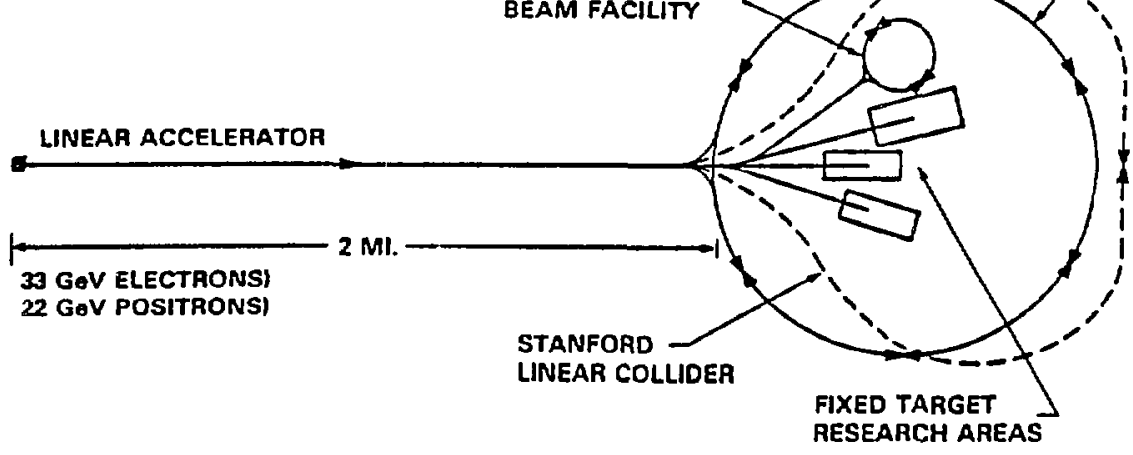


date some of the recently observed anomalies in these processes, has also been completed. An off-axis injector has been constructed to give a relatively cheap way of generating 3- to 4-GeV eloctrons. This improvement is motivated mainly by the approved nuclear yhysics program but will also provide an economical way of filling SPEAR.

b. PEP Program at SLAC. PEP is a high energy electron-positron storage ring, wich can provide collisions at reaction energies between 8 and $30 \mathrm{GeV}$. Since it started operation in 1980, its luminosity has been steadily improved and has recently reacised $3 \times 10^{31} \mathrm{~cm}^{-2}$ $\mathrm{sec}^{-1}$. The parameters of PEP are very similar to those of the PETRA storage ring at DESY in Hamburg, West Germany, and during their initial operation the experiments at these two storage rings addressed the same basic physics questions. Subsequently their physics goals have diverged somewhat. The staff at PETRA has pursued added rf capability to reach the highest energies possible ( $45 \mathrm{GeV}$ total centerof-mass energy), hoping to exceed the threshold for production of the postulated top quark; while workers at PEP have been capitalizing on its high luminosity to obtain very accurate measurements at a single energy ( $29 \mathrm{GeV}$ total energy).

Planned future upgrades of PEP include further increases in Iuminosity by micro-beta beam focusing insertions as well as corresponding improvements to the detectors. Five general purpose detectors have been operating at PEP: Mark II, a high resolution spectrometer (HRS), a detector oriented around a time projection chamber (TPC), a magnetic calorimeter detector (MAC), and a detector oriented around a large threshold Cerenkov counter (DELCO). Recently, a specialized detector to look for anomalous single photons (hSP), covering full $4 \pi$ solid angle, has been operating in the sixth interaction region to look for missing energy and transverse momentum in the search for possible long-lived non-interacting neutral paricles postulated in supersymetric theories. Two specialized experiments have already been completed: a free quark search and a monopole search, both with negative results.

These diverse detectors allow a correspondingly diverse physics program at PEP. In hadronic physics, the emphasis is on detailed QCD tests looking at quark and gluon jets, particle correlations in multi-jet events, and studies of the hadronization process for both the light and heavy quarks. The electroweak sector includes measurements of the neutral weak coupling constants of quarks and leptons by studying forward-backward asymetries and determinations of the weak mixing angles from B-meson decays. Recent upgrading of several detectors with precision vertex chambers has allowed precise determination of lifetimes of the tau leptons, charmed states, and B-quark states. An extensive two-photon physics program is under way, and a dedicated experiment to search for the photino, the photon's partner in supersymetric theories, is now in progress.

The recent planned improvements in luminosity will allow several years of productive research with high statistics experiments.

c. SPEAR Program at SLAC. SPEAR is a medium energy electronpositron storage ring with reaction energies between 2.5 and $8 \mathrm{GeV}$. 
It has been operating for more thin a decade, but its physics program continues to be very productive. Its main value is that it is the unique $e^{+} e^{-}$facility in the world in the $3-$ to $5-\mathrm{GeV}$ total energy region-the region that allows study not only of charmonium spectroscopy but also of the production and decay of various charmed states in relatively clean circumstances. Because different studies ( $\psi$ decay modes, $D$ mesons, $F$ mesons, charmed baryons) require different energies, they cannot be performed concurrently.

SPEAR has two interaction regions, but, since the Crystal Ball detector was moved to DORIS at DESY, only one has been occupied. It serves a general purpose detector known as Mark III. This detector has been optimized for study of the SPEAR energy region and features good efficiency for low energy gamma rays, a precision time-of-flight system to separate pions from kaons and protons over most of the kinematic range, and good charged particle momentum measurement. It has large solid-angle coverage and can be used to reconstruct a large fraction of charmed particle decays.

Recentiy SPEAR has been Iunning half time as a dedicated synchrotron radiation source for the Stanford Synchrotron Radiation Laboratory, and it will continue to do so in the forseeable future. The Mark III program is expected to remain productive at least until 1988.

d. SLAC Linear Collider (SLC). Construction of the SLC began in October 1983. Its justification is twofold. First, it provides for the development of techniques essential for establishing the tachnical feasibility of linear electron-positron colliders, whose energies could reach well beyond those feasible with circular colliders in view of projections that the cost of linear colliders rises linearly with the $e^{+} e^{-}$center-of-mass energy whereas that of storage ring colliders rises approximately as the square of this energy. Second, the SLC is designed with a reaction energy high enough to copiously produce directly the neutral intermediate boson, the $z^{\circ}$, recently discovered at CERN. The scheme (Figure 18) will bring into collision highintensity micron-sized bunches of electrons and positrons, each beam with an energy up to $50 \mathrm{GeV}$ for a total center-of-mass energy of up to $100 \mathrm{GeV}$ in the $\mathrm{e}^{+} \mathrm{e}^{-}$system. These bunch pairs arrive at the collision point from opposite directions, at the pulse rate of the SLAC Iinear accelerator ( 120 per second, eventually to be increased to 180 ). After the beams collide, the residual electrons and positrons are disposed of in beam dumps. SLC will also be capable of collisions witr polarized beams, and its very small beam size will allow increased opportunities for close-in vertex detectors and hence good conditions for studies of short-lived particles. It is scheduled to be completed in the second quarter of FY 1987.

The topics to be studied at the SLC include $Z^{\circ}$ production and decays (mass, width, decay modes and branching ratios, number of flavors, number of generations of neutrinos); study of top quarks by directly producing the vector tEE bound state (toponium); search for new particles such as the Higgs, particles predicted by supersymetry or technicolor, heavy leptons, and other new heavy quarks; QCD tests by studying quark and gluon jet:s; and study of the mixing between the 


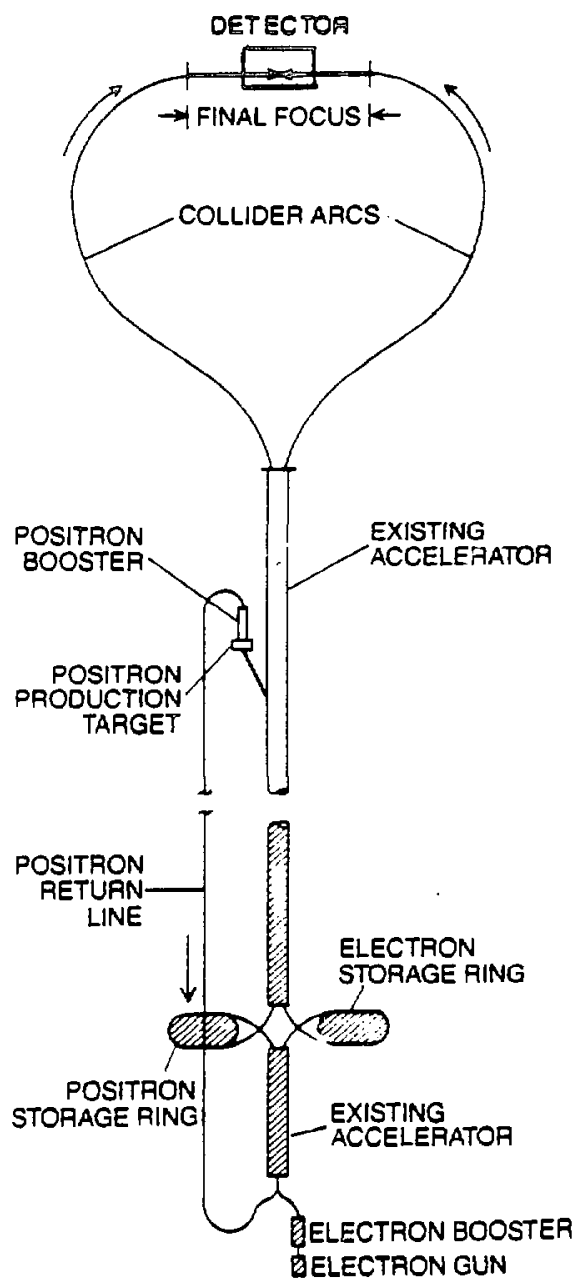

Figure 18. General layout of the SLAC Linear Collider (SLC): the injection system (electron gun and boosters), storage (damping) rings which serve to reduce the size and energy spread of the electron and positron bunches by radiation damping, the existing linac which accilerates bunches of electrons and positrons to $50 \mathrm{GeV}$, and the transport and final focusing systems which bring micron-sized bunches of electrons and positrons into head-on collisions. The positron target and booster use electron bunches to produce positrons for injection into the front end : $\varepsilon$ the linac.

different quark $f l$ avors ( $K-M$ mixing angles). These objectives are similar to those of LEP at CERN. The SLC, al though lower in energy than LEP, has a design luminosity $\left(16 \times 10^{30} \mathrm{~cm}^{-2} \mathrm{sec}^{-1}\right)$ that is comparable with that of LEP, but it is due to have its first physics run about two years earlier.

The SLC has two technical advantages over storage rings. First, detectors can be placed closer to the interaction region than in a storage ring. This allows the position of secondary vertices to be measured with high accuracy, improving the measurement accuracy of short 1 ifetimes and increasing the efficiency for tagging heavy quark decays. The second advantage is the ability to produce longitudinally polarized electron beams. This allows the $Z^{\circ} s$ to be produced with a tunable polarization, aiding in the neasurement of electroweak interaction parameters and the determination of new particle properties. The ability to produce a polarized electron beam and to measure its polarization will probably not be available until 1989.

During SLC commissioning and initial stages of operation, the Mark II detector (suitably upgraded) will be used in the single interaction region. This has the advantage that physics results can be obtained relatively quickly with $a$ detector that is well understood because of its previous operation at SPEAR and PEP. Fabrication of 
a general purpose detector, designated SLD, designed expressly for this energy range, is underway so that the full physics potential of the SLC will be exploited. The characteristics of the detectors for LEP and SLC are compared in Table VII.

\section{Fermi National Accelerator Laboratory (Fermilab)}

Until the fall of 1983 the physics program at Fermilab used beams produced by a $400-G e V$ proton synchrotron. The Energy Saver superconducting magnet ring (also called the Tevatron), which is expected eventualiy to reach particle beam energies up to $1 \mathrm{TeV}$, has recently come into operation. (Al though the Tevatron has a $1-\mathrm{TeV}$ capability, it is being operated at $800 \mathrm{GeV}$, with the limitation on energy being due to cryogenic capacity and the presence in the ring lattice of a small number of field-limited magnets.) The so-called Main Ring synchrotron, now operated at $150 \mathrm{GeV}$, serves as the injector for the Energy Saver. The $1-\mathrm{TeV}$ beam capability will be used in two different modes of operation: colliding 1-TeV proton and antiproton

TABLE VII

Summary of the New $e^{+} e^{-}$Detectors

\begin{tabular}{|c|c|c|c|c|c|c|}
\hline & $\begin{array}{c}\text { ALEPH } \\
\text { (LEP) }\end{array}$ & $\begin{array}{c}\text { DELPHI } \\
\text { (LEP) }\end{array}$ & $\begin{array}{l}\text { L3 } \\
\text { (LEP) }\end{array}$ & $\begin{array}{l}\text { OPAL } \\
\text { (LEP) }\end{array}$ & $\begin{array}{l}\text { Mark II } \\
\text { (SLC) }\end{array}$ & $\begin{array}{l}\text { SLD } \\
\text { (SLC) }\end{array}$ \\
\hline $\begin{array}{l}\text { Vertex } \\
\text { Detector }\end{array}$ & $\begin{array}{c}\text { chamber }+ \\
\mu \text {-strips }\end{array}$ & $\underset{\mu \text {-strips }}{\text { chamber }}+$ & TEC & chamber & $\begin{array}{c}\text { chamber + } \\
\text { possible } \\
\mu-s t r i p s \\
\text { or CCD's } \\
\end{array}$ & CCD's \\
\hline $\begin{array}{l}\text { Tracking } \\
\text { Device }\end{array}$ & $\begin{array}{c}\text { TPC } \\
1 \mathrm{~atm} \\
r=1.8 \mathrm{~m} \\
n=300\end{array}$ & $\begin{array}{c}\text { TPC } \\
1 \mathrm{~atm} \\
r=1.2 \mathrm{~m} \\
+ \text { outer } \\
\end{array}$ & - & $\begin{array}{c}D E \\
4 \mathrm{~atm} \\
r=1.6 \mathrm{~m} \\
n=160\end{array}$ & $\begin{array}{c}D C \\
1 \mathrm{~atm} \\
r=1.5 \mathrm{~m} \\
n=72\end{array}$ & $\begin{array}{c}D C \\
i \mathrm{~atm} \\
r=1.0 \mathrm{~m} \\
n=80\end{array}$ \\
\hline Coil & $\begin{array}{l}\text { s.c. } \\
1.5 \mathrm{~T}\end{array}$ & $\begin{array}{l}\text { s.c. } \\
1.2 \mathrm{~T}\end{array}$ & $\begin{array}{l}\text { warm } \\
0.5 \mathrm{~T}\end{array}$ & $\begin{array}{l}\text { warm } \\
0.4 \mathrm{~T}\end{array}$ & $\begin{array}{l}\text { warm } \\
0.5 T\end{array}$ & $\begin{array}{l}\text { warm } \\
0.6 \mathrm{~T}\end{array}$ \\
\hline $\begin{array}{c}\text { Tracking } \\
\text { Resolution } \\
\end{array}$ & $0.13 \% p$ & $0.15 \% p$ & $\begin{array}{c}0.03 \% \text { p } \\
\mu \text { oniy }\end{array}$ & $0.15 \% p$ & $0.12 \% p$ & $0.73 \% p$ \\
\hline Particle Id & $\begin{array}{l}d E / d x \\
4.5 \% 0\end{array}$ & $\begin{array}{c}d E / d x+ \\
\text { RICH }\end{array}$ & - & $\begin{array}{l}d E / d x \\
3.5 \% 0 \\
\end{array}$ & $\begin{array}{l}d E / d x \\
7 \% a\end{array}$ & $\mathrm{RICH}$ \\
\hline EM calor. & Pb-gas & HDPC & BGO & Pb glass & $P b-L A$ & U.LA \\
\hline EM resolution & $18 \% / \sqrt{E}$ & $18-20 \% / \sqrt{E}$ & $1 \%$ & $6.5 \% / \sqrt{E}$ & $12 \% / \sqrt{E}$ & $8 \% / \sqrt{E}$ \\
\hline $\begin{array}{c}\text { Hadron cal. } \\
\text { Resolution }\end{array}$ & $100 \% / \sqrt{E}$ & $100 \% / \sqrt{E}$ & $\begin{array}{c}50 \% / \sqrt{E} \\
+10 \% \\
\end{array}$ & $100 \% / \sqrt{E}$ & - & $45 \% / \sqrt{E}$ \\
\hline Muon Det. & full & full & high res & full & $55 \%$ & full \\
\hline
\end{tabular}

Key: TEC - Time Expansion Chambar

CCD - Charged Coupled Devica

TPC - Time Projection Chambisr

DC - Drife Chamber

RICH - Ring Imaging Cherenkov Detector

HDPC - High Density Projection Chamber

LA - Liquid Argon

(p in GeV) 
beams (Tevatron I project), and fixed-target experiments with $1-\mathrm{TeV}$ protons (Tevatron II project).

a. Tevatron II Program at Fermilab. The Tevatron II fixed-target program (Figure 19) provides for extraction of a $1-\mathrm{TeV}$ beam from the Energy Saver ring; upgrading the switchyard, external proton beam lines, target stations, and existing experimental halls for 1-TeV operation; and four new secondary beams including a high flux muon beam, a wideband photon beam, a polarized proton beam, and a meson-west pion beam. The proton beam extraction and proton beam lines in the switchyard are now in operation, and the entire project is expected to be completed in 1986. The increased proton beam energy will provide secondary particle beams with higher energy and increased intensity per pulse; however with reduced cycle rate. In addition, the use of superconducting magnets substantially improves the operating performance, in particular the beam spill time. The result is that the duty cycle (i.e. beam-on-target vs. cycle time) is as high as $30 \%$. This is a substantial improvement over the non-superconducting machine (Main Ring), where the duty cycle was 8 to $10 \%$.

The fixed-target experimental program covers a broad spectrum. Measurements of leptons produced at large transverse momentum have resulted in interesting comparisons with quark model predictions as well as the discovery of the upsilon particle. These programs will continue to higher energies and will be broadened to include beam particles other than protons and final state particles other than leptons. Studies of hadron jets and direct photons are also being emphasized. Measurements with a new polarized proton beam and with polarized targets will be possible.

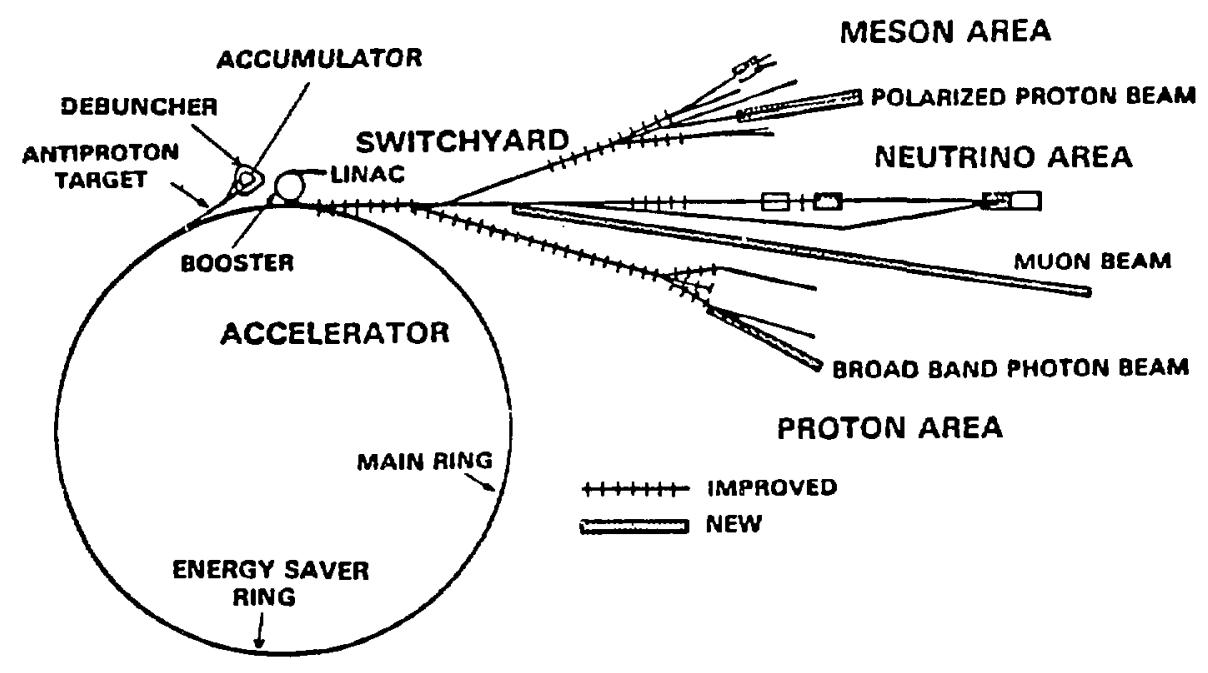

Figure 19. The Tevatron facility at Fermilab: the 6-km-circumference Main Ring and superconducting (Energy Saver) ring; the switchyard and external beamlines for fixed-target operation ( $\mathrm{TeV}$ II); the antiproton source (antiproton target, accumulator, and debuncher) for the colliding $\mathrm{p} \overline{\mathrm{p}}$ operating mode ( $\mathrm{TeV} \mathrm{I}$ ); and the Main Ring injector complex (1inac and booster). 
Several experiments on studies of charm and bottom production will use various novel techniques in different secondary beams. of special interest are efforts to develop vertex detectors and fast online processors to extract the events containing decays of heavy quarks, providing measurements of the Kobayashi-Maskawa $(K-M)$ mixing angles.

A series of experiments using neutrino and muon beams will extend the measurements of nucleon structure functions by about a factor of three in momentum transfer $\left(Q^{2}\right)$. This may turn out to be important because of the present confusion in the $Q^{2}$ evolution of structure functions (the value of $\Lambda_{Q C D}$ is smaller when it is obtained from data at higher $Q^{2}$ ). The neutrino experiments will be capable of more precise measurements of neutral current couplings, which can be compared with measurements from $\mathrm{e}^{+} \mathrm{e}^{-}$colliders operating at the $\mathrm{Z}^{\circ}$ mass. The muon experiments will study jet fragmentation and atomic number dependence at very high $Q^{2}$. The latter has recently been of considerable interest. Study of rare phenomena, like multi-lepton production by both muons and neutrinos, with much higher event rates at higher energies will improve understanding of these processes. Holography will be used in the 15-ft bubble chamber to improve track resolution and thus permit easier observation of heavy quark decays.

Some of the many phenomena from lower energies that are still not understood will be subjects of Tevatron II experimentation. A high statistics measurement of the electron asymetry parameter in the $\beta$-decay of $\Sigma^{-}$should corroborate or remove the present large discrepancy betueen this quantity as inferred from existing data and the prediction of the Cabibbo model. Precision measurements of CP violation parameters will be made at levels where effects predicted by certain classes of theories should become manifest. CP violation may be observed in channels where it has not yet been seen.

b. Tevatron I Program at Fermilab. The Tevatron I project provides a proton-antiproton collider with a center-of-mass energy of up to $2 \mathrm{TeV}$. Specifically it includes (1) additional refrigeration and radio-frequency accelerating structures so that the superconducting Energy Saver ring can operate at $1 \mathrm{TeV}$ in a storage ring mode; (2) an antiproton source (Figure 20) and accumulation system; and ( 3 ) two large experimental areas suitable for detectors to study the results of very high energy proton-antiproton collisions.

According to the present schedule, the Fermilab collider program will furnish up to $2-\mathrm{TeV}$ p $\bar{p}$ collisions for physics experiments by 1986-87 with a luminosity of $10^{30} \mathrm{~cm}^{-2} \mathrm{sec}^{-1}$. Two major collision regions are being planned: one at $B O$, where the construction is complete, and one at DO, which will be finished in 1987. A large general purpose detector (CDF) is now being fabricated for the BO area. Fabrication of a complementary detector for the DO area is under way. The first round of experiments is expected to run for four, five years with an integrated Imminosity of up to $10^{37} \mathrm{~cm}^{-2}$ or $10^{4} \mathrm{nb}^{-1}$ per year of running. The Tevatron I program will divide the available time with the Tevatron II fixed-target program, in a manner similar to CERN operation. 


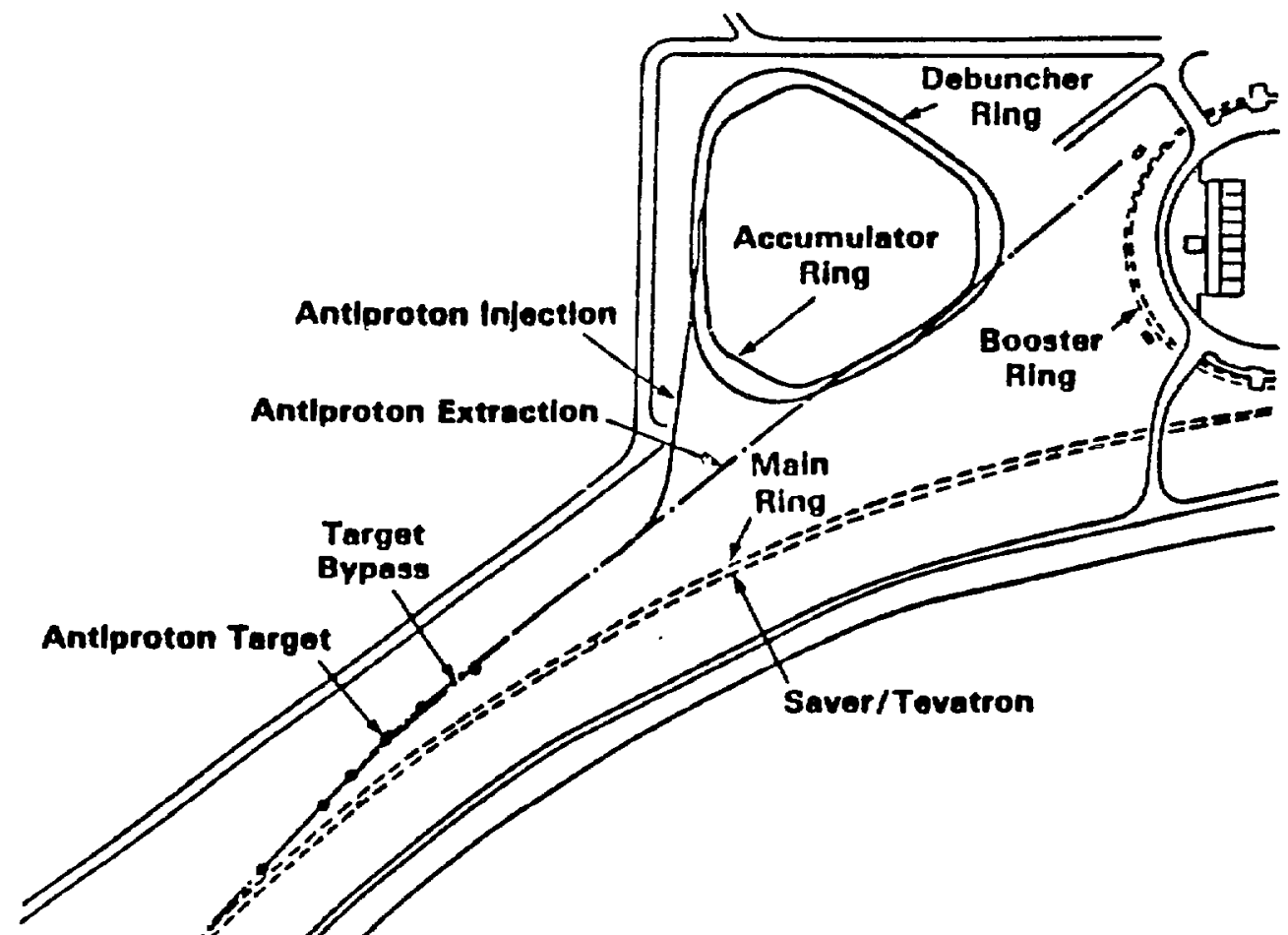

Figure 20. Tevatron I antiproton source.

The CDF detector employs a large solenoidal magnet which contains track chambers surrounding the beam pipe. The superconducting solenoid magnet is enclosed within a lead-scintillator electromagnetic calorimeter which, in turn, is enclosed in an iron-scintillator hadron calorimeter. The calorimeters utilize a highly segmented tower geometry in order to define the energy and direction of a jet. It will be possible to detect electrons and hadrons emerging from the interaction point at an angle of $l^{\prime \prime}$ or greater. Muon detection is provided over part of the solid angle. The construction of the detector will be complete in late 1986. The partially completed detector will be placed in the Tevatron Collider during a September 1985 beam test, and it will be used again in another colliding beams test planned for 1986 .

Unlike CDF, the DO detector has no central magnetic field, but emphasizes optimum muon and electron identification and minimizes missing transverse energy resolution. Its designers have chosen highly segmented uranium--iiquid argon calorimetry, utilizing projective geometry. The calorimeters will measure the energy of particles which emerge from the interaction point at angles $>1^{\circ}$ with respect to the beam axis. A special effort is being made to reduce both the statistical errors and the systematic errors in the measurement of electron and hadron energies. Electron identification is achieved through measuring shower development in the calorimeter and the ionization deposited in a transition radiation detector. The detector also has a magnetized iron muon detector covering nearly 4 ateradians. On the basis of the current plan the detector can be completed in 1990, with a staged turn-on beginning in 1989 .

The Tevatron I program has two main features providing physics opportunities. The primary one is the $2 \mathrm{TeV}$ available for producing 
new heavy particle states. This high energy gives Tevatron I a position unique in the world for the 1986-92 period. An added feature is. that the successful construction and operation of an antiproton source will enhance the development of that complex technology in the U.S. The anticipated annual yields at the Tevatron of standard model. intermediate bosons that can be exploited for detailed study are $2 \times 10^{5}$ Wt and $6 \times 10^{4} \mathrm{Z}^{\circ}$. Examples of the discovery potential of the new machine are $s 400-\mathrm{GeV}$ mass for new intermediate bosons, $s 100-\mathrm{GeV}$ mass for new heavy quarks, and $s 300-\mathrm{GeV}$ transverse momentum for QCD jets. During the late $1980 \mathrm{~s}$ this program is expected to occupy about $400 \mathrm{U}$.S. high energy experimental physicists.

\section{Cornell Laboratory for Nuclear Studies}

The Cornell electron-positron storage ring (CESR), supported by the National Science Foundation, has been operating since 1979, principally in the reaction energy range 9 to $12 \mathrm{GeV}$, although higher energies are possible. It has two interaction regions, one occupied by a large general purpose magnetic detector ( $C L E O$ ) and the other by a nonmagnetic detector for high resolution electromagnetic calorimetry (CUSB).

The CESR energy range is ideal for studying the bottom quark. Since CESR came into operation, the CLEO and CUSB experiments have studied the first six ${ }^{3} \mathrm{~S}$ states and the first two triplets of ${ }^{3}$ ? states in the bb quarkonium system. They have measured the dilepton and hadron decay widths of the first three ${ }^{3} \mathrm{~S}$ upsilons as well as the branching ratios for $\pi \frac{\pi}{3}$ transitions among them and the radiative transitions involving the ${ }^{3} \mathrm{p}$ states. The fourth upsilon has served as a "B-meson factory," allowing studies of the weak decays of the b quark: semileptonic branching ratios, multiplicities, inclusive $K, D, p$, and lepton yields, and exclusive modes. The results have confirmed the standard six-quark model and have established the $b \rightarrow c \rightarrow s$ cascade as the dominant decay mode.

An important gap in the $b \bar{b}$ bound state spectroscopy is the ${ }^{1}$ $n_{b}$ state which should be accessible from the upsilon states by a sequence of hadronic and radiative transitions. The systematic reconstruction of upsilon decay modes permits possible discoveries of glueballs, Higgs, axions, supersymetric partners, and perhaps other surprises. A thorough study of $B$ decays will be required to measure the generalized Cabibbo weak mixing angles, BOBO mixing, $C P$ violation, and rare modes such as $B \rightarrow \tau V$. Of the higher mass b-flavored hadrons, $B^{*}, B_{s}, \Lambda_{b}$, etc., only the $B^{*}$ has been observed.

The studies of the $b$ quark at CESR are important for progress in understanding both strong and weak interactions. Because the $b$ quark is so massive, the $b \bar{b}$ system is especially amenable to quantitative QCD predictions. The weak decays of the $b$ quark are unique in that they permit measurement of branching ratios which depend significantly on the weak mixing angles other than the original Cabibbo angle; the $\mathrm{B}^{\circ} \overline{\mathrm{B}} \circ$ system (or $\overline{\mathrm{B}}_{\mathrm{S}} \mathrm{B}_{\mathrm{S}}$ ) may provide the only chance to observe $\mathrm{CP}$ violation outside the $\mathrm{K}^{\mathrm{O}} \mathrm{K}^{\circ}$ system. One can hope that this work will lead to insights as to why there are three generations of quarks and leptnas. 
The future CESR physics program requires significant increases in quantity and quality of data for the following reasons. Relative to cross sections in the charmonium region, b $\vec{b}$ cross sections are suppressed because of quark charge and because of increased energy. Moreover, in the $\mathrm{b} \bar{b}$ case there are more states and more decay modes and, therefore, smaller branching ratios. The CESR luminosity improvement program is needed to provide at least a tenfold increase in rates. In addition, an extensive upgrade of the CLEO detector is under way and will provide significantly improved charged particle tracking and particle identification as well as photon energy resolution. The present CUSB experiment has been upgraded by improving the photon energy resolution.

\section{Non-Accelerator Program}

The U.S. High Energy Physics Program has traditionally supported a modest but diversified program of non-accelerator physics experiments. Recent predictions from grand unified theories have rekindled interest in searches for nucleon instability, heavy primordial magnetic monopoles, neutron oscillations, and other new phenomena. In fact, a stimulating merging of the interests of traditional high energy physicists with those of cosmic-ray physicists, astrophysicists, astronomers, and cosmologists is taking place. Non-accelerator experiments in these areas include searches for gravity radiation, neutrinoless double beta decay, neutrino mass, neutrinos from gravitational collapse, proton decay, and magrietic monopoles; studies of atmospheric neutrinos and neutrino oscillations, extraterrestrial and extragalactic cosmic rays, and gamma-ray and neutrino astronomy up to several $\mathrm{TeV}$ in energy; and solar neutrino measurements.

It is important to maintain U.S. effort in these areas. These experiments add significantly to the diversity of the U.S. high energy physics program and are especially desirable in the present theoretical climate where seeing what lies beyond the standard model is of prime importance. Most have direct relevance to the studies at high energy accelerators, and some explore physics on an energy scale beyond that attainable with accelerarors. Moreover, constraints on new theoretical ideas often come from exp-riments done for an entirely different purpose. For example, measureciants in $x$-ray astronomy put constraints on monopole catalysis of nucleon decay, and solar neutrino flux measurements put limits on the rate of monopole catalysis in the sun.

During the last few years, the proton decay program has been especially strong and diversified. Recent results from the large water Cerenkov experiment (Irvine-Michigan-Brookhaven collaboration) have put into question the simplest grand unified theories, and refined measurements continue to come from new data. The future tracking calorimeter experiment (Soudan II) should provide information that is complementary to that from the water Cerenkov detectors. Reactor neutrino experiments provide a unique means of studying neutrino masses and lepton mixing, because of the high fluxes and 1 ow energies of reactor neutrinos. 
During the next decade the non-accelerator elementary particle program should continue to play a vital role, complementing accelerator-based research. It not only provides a means of glimpsing an energy scale inaccessible to currently conceivable accelerators, but also connects the field of high energy physics with many othrs branches of physics.

\section{University-Based Research}

Modern high energy physics research started on the campuses of II.S. universities soon after the end of World War II, when a large group of scientists became free to pursue basic research. These scientists, with funding from the Federal Government, enabled maior universities, from coast to coast, to establish particle accelerators on campuses. As the machines reached the $\mathrm{GeV}$ energy range and their costs multiplied, a growing number of universities could not afford their own particle accelerator. Nevertheless, this field of research required higher energy accelerator facilities. The result was the arrangement now in effect: accelerators are built and operated at a few large national laboratories, (managed by individual or groups of universicies), and the experiments at them are done primarily by university-based scientists--in the user mode (see Figure 21 and Table VII).

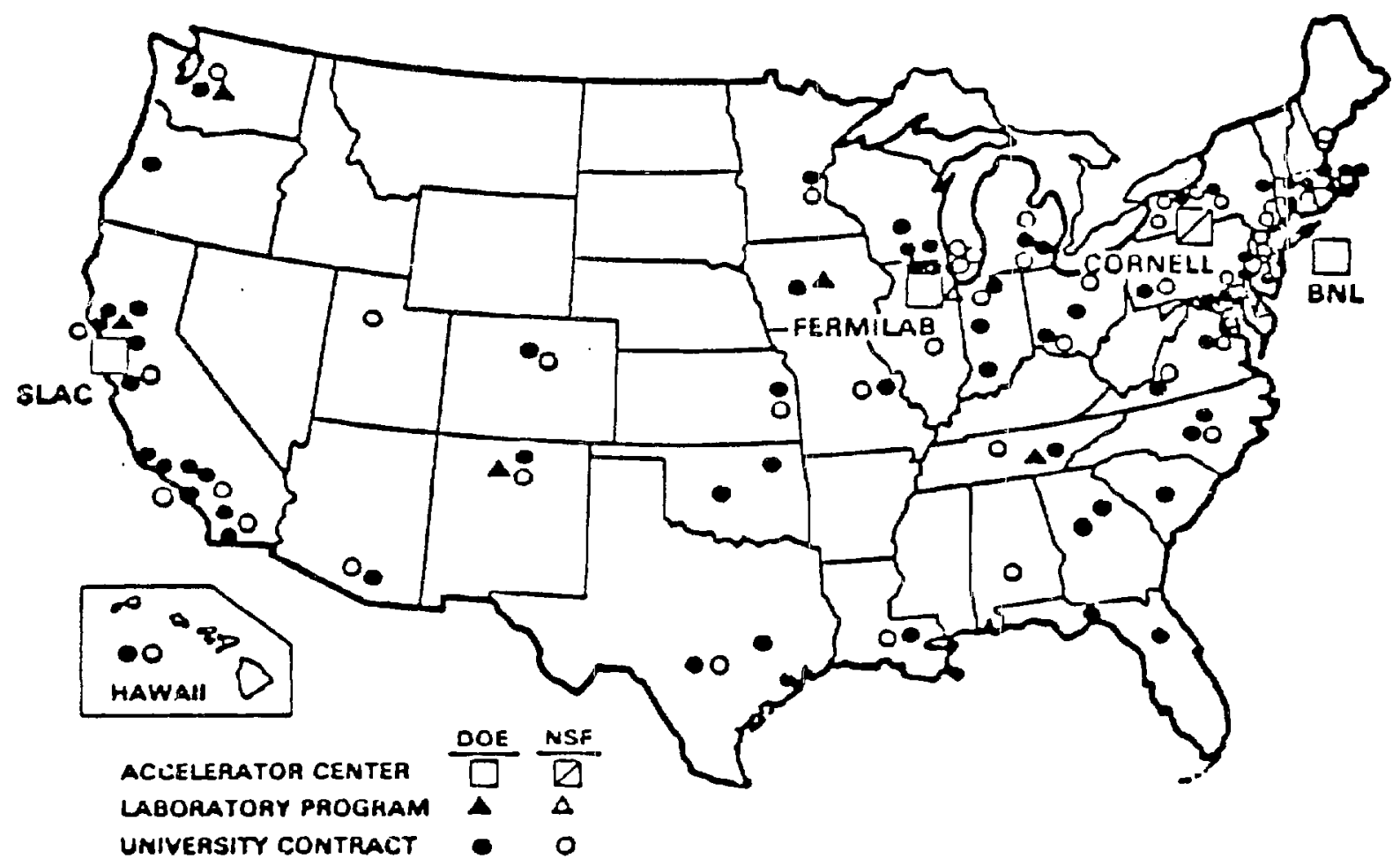

Figure 2!. The location of participants in the U.S. High Energy Physics Program as of 1983. Accelerator centers, national laboratories, and universities supported by DOE and NSF are indicated. 
Table VIII liser group mode

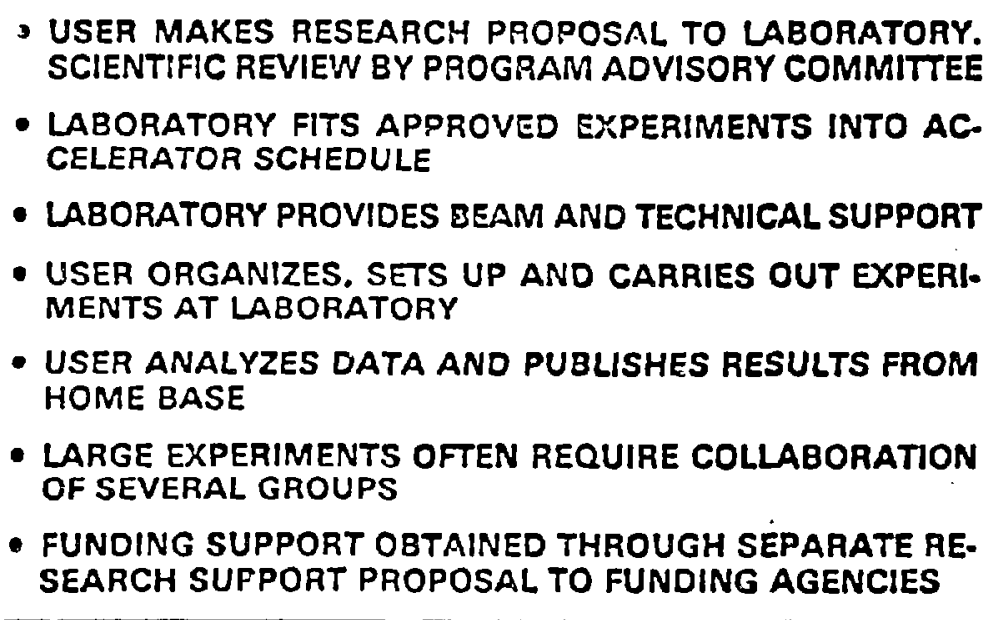

This arrangement has been extremely successful, and Western Europe has also adopted close coupling between large accelerator laboratories and universities.

The user mode allows the construction of facilities far larger than could be supported by any one university, and yet maintains the major advantages of university-based research. Because high energy physics is recognized as being of fundamental importance by the general public as vell as by scientists, universities have made an effort to attract and hold the best scientists (both from the U.S. and from abroad) and have thus remained the principal source of advances in hoth theory and experimental work.

A great advantage of keeping scientists at universities while providing them kith access to forefront research centers is that they maintain their association not only with the university staff but also with the students, which is vital to the continuation of scientific acivance. High energy physics needs new entrants, but even students who do not stay in this field benefit from contact with it.

An important interaction on campus is that between high energy theorists and experimentalists: the former learn which ideas might be explored by experiment, and the latter learn which experiments are needed for theoretical progress. Also important is the flow of ideas to and from other disciplines. High energy physics results have found their way into solid state theory, molecular and neurobiology, medical physics, plasma physics, synchrotron radiation facilities, cosmology, and astrophysics. In turn, computer science, solid state physics, cosmology, laser physics, the physics of gases, and electronic engineering have advanced high energy physics.

In a university environment, scientists are free to change the emphasis of their research. New opportunities quickly find energetic proponents, and this fosters the timeliness of research trends. Since peer review panels and program advisory committees at the accelerator laboratories include many university scientists, the high energy physics research community can adapt quickly to new ideas. 
During the academic year 1982-83, a Technical Advisory Committee on University Programs (TACUP) appointed by the High Energy Physics Division of DOE carried out a comprehensive review of the entire DOEsupported university-based program in high energy physics in order to assess the quality of the individual university contracts funded by DOE. TACUP was also asked to comment on the balance between the various components of the DOE High Energy Physics Program and to report opinions based on its review of the separate contracts and on comments solicited from the high energy community.

TACUP was asked to review 82 contracts comprising 64 separately funded theory tasks at 53 institutions, and 107 experimental tasks at 58 institutions. Eight panels, three for theory and five for experimental work; were appointed, and all used similar quality criteria. The evaluation was based on (1) a dossier submitted by the task leader, and (2) a conference between the panel and the task leader, with a site visit for larger contracts. Final reports, providing a quality evaluation for each of the 171 tasks, were submitted to DOE in May 1983.

Such a comprehensive review of all university-based DOE contracts simultaneously has not been carried out before, and the TACUP review provides a unique overall picture. The main conclusion from the individual task evaluations is that, with few exceptions, the university-based contracts produce research of very high quality. The high energy physics community is proud of the scientific achievements of the last decade and eager to deal with the new and deeper questions which these successes have suggested. The individual research programs, even though they address a bewildering array of topics, are well focused on issues of current scientific importance, and competition to obtain significant results is keen.

The High Energy Physics Program has subdivisions beyond theory and experiment. Experiments can be speculative or can be designed to add to knowledge in a predictable way; they can depend on accelerators-U.S. or foreign-- or be independent of them. Given the totality of funds available, TACUP concluded that the allocation of resources to these various program components was reasonably matched to the opportunities in each area.

Enthusiasm in the high energy community continues to be high, but the TACUP panels reported a significant level of concern. The number of U.S. accelerator facilities that can support front-rank experiments has been decreasing steadily. For example, only three fully instrumented collider interaction regions, two at Tevatron $I$ and the other at SLC, will permit access to new energy regimes at the end of the decade. The number of U.S. research teams planning experiments in Western Europe is increasing. Many university scientists are concerned that the shrinking opportunities foreseen in the $1990 \mathrm{~s}$ will discourage young researchers from committing their careers to the field. TACUP found the question of the development of frontier facilities in the U.S. in the $1990 \mathrm{~s}$ with adequate opportunities for university research groups to be a matter of great urgency.

Over the years, funding 1 imitations have not allowed the full exploitation of the existing U.S. accelerators, resulting in an increase in the already long time spans between initiation and completion of ex- 
periments and in a reduction in the number of experiments performed. TACUP recommended, as have many others, that operating funds earmarked for accelerator operation be increased until these large facilities are fully exploited.

Universities have opportunities for input into the decisionmaking process, but there is probably a need to develop further effective channels for participation in shaping the High Energy Physics Program. The Snowmass meeting of 1982 was useful in this respect, and workshops under APS Division of Particles and Fields sponsorship should be continued, with broad university participation.

TACUP did not study the program funded by the Advanced Technology $R$ and $D$ Branch of DOE, but many of the task leaders told the panels that research on advanced accelerator concepts is not being pushed as vigorously as it should. This would be an appropriate activity for universities since accelerator laboratories must be concerned also with more immediate improvements. The advisability of additional funding for universities for advanced accelerator research should be determined.

TACUP found that opportunities for young physicists to enter faculty positions has somewhat improved. The staffs of many large and medium-size research tasks now include a tenure-track faculty member hired in the last six years. The DOE Outstanding Junior Investigator (OJI) program has contributed significantly to this improvement. Since its inception in 1978, more than 50 theorists and experimentalists have been OJIs.

Since 1974, DOE support of its university-based physics program has been essentially constant at $\$ 53$ million per year (in 1982 dollars), but indirect costs have increased by about $10 \%$, and the experiments have become much more complex and costly. The TACUP panels found most tasks to be handicapped by serious funding constraints. On the theory side, funding shortages impede the training of students and postdoctorals and restrict travel and visitor exchanges. On the experimental side, universities have infrastructures that are deteriorating and apparatus that is no longer up-to-date, and few of them can maintain a stable group of engineers and technicians. Thase trends should be reversed by additional funds, especially ior equipment modernization and for support of $R$ and $D$ projects not directly tied to approved experiments.

The use of computers by theorists is burgeoning. A major new development is their application to clarifying basic mathematical ideas such as lattice gauge theories. This requires computers that not only are large but also have special characteristics designed to handle such calculations efficiently. The pressing needs in this area cannot be met by a small fractional increase in the theory hudget, and DOE should set up a panel to study computer needs and estimate budget requirements.

The assignment of VAX (or eqivalent) computers to a number of experimental tasks has been most welcome. The need for more computers-of higher power and greater variety--will continue and should be given high priority, with at least one VAX (or equivalent) planned per mediumsize research team, plus one located at the experiment and shared by the collaborators. The panels also found a large unmet need for 
"networking," i.e., communication between the compueers of a collaboration situated on various compuses, even though a few large collaborations are developing their own systems. DOE $t \cdot a$ convened a panel to evaluate various networking approaches, and commendations to the funding agencies are continuing.

TACUP emphasized the importance of graduate and postdoctoral training in the High Energy Physics Program. Summer study institutes have in fact been initiated for the training of postdoctoral theorists and experimentalists. Such institutes provide discussions of important current work, produce relevant pedagogical literature, and enhance interactions between students. Many graduate students doing experimental work at acceierator laboratories must interrupt or discontinue their formal education, and this is a growing problem as the time needed at the accelerator site lengthens. It would be helpful if the accelerator laboratories greatly increased the number of minicourses and lecture series to compensate for the formal courses on campus.

TACUP found the relations between the university users of acceleracors and the laboratory staffs to be excellent and mutually supportive but suggested that both groups would benefit from more frequent exchanges of personnel than at present and encouraged DOE support for such exchanges.

As a result of this comprehensive review, corrective actions were taken with respect to some 10 to $15 \%$ of the DOE-supported university contracts, including some terminations.

III. INTERNATIONAL COOPERATION IN HIGH ENERGY PHYSICS

\section{A. General}

International collaboration is exceptionally wel1-suited to high energy physics and has long been effective and mutually beneficial. Shared use provides access t:o unique facilities in one nation which are often complementary to those in another. It provides research opportunities that would otherwise not be available because of the worldwide distribution of unique facilities. Shared use has proved to be mutually beneficial, and the cash flow, averaged over the years, is wel1 balanced between cooperating nations and entities having major facilities.

Coordination of plans for constructing major new facilities, and international participation in research, including joint fabrication of large-scale equipment, is useful for progress on an international scale. However, no formal joint decision-making mechanism has been set up, and no joint construction venture (if CERN is considered a single participant) has been undertaken. In another area, some nonaccelerator experiments require unusual geophysical conditions such as very deep mines or a specific geomagnetic latitude, and these depend on international access, the history of which has generally been positive.

In the late 1950s and early 1960s, when the U.S. facilities (and their support) were better than those elsewhere, individual and institutional participation by foreign scientists in the U.S. was 
high, but few U.S. physicists worked abroad. The balance improved in the late $1960 \mathrm{~s}$ and early 1970s with the growth of CERN and DESY in Europe. The past ten years have seen extensive U.S. institutional involvement in both the ISR at CERN and PETRA at DESY. There is now

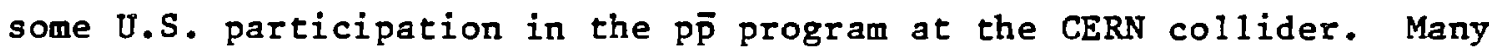
U.S. groups have made a strong commitment to work at LEP and TRISTAN, and others have expressed significant interest in doing experiments at HERA located at DESY. There is also U.S. participation at the DORIS storage ring at DESY and at LEAR, the low energy $p \bar{p}$ ring at CERN.

The fraction of the university high energy budget spent for work using unique facilities outside the U.S. has recently risen to $>10 \%$ with the increasing expenditures for the LEP detectors. A formal policy, initiated by the International Committee on Future Accelerators (ICFA) and adopted by all worldwide participating laboratories, specifies that scientific merit and feasibility of experiments shall be the sole criterion for acceptance of programs and experiments. This provides the basis for a reasonable "trade balance."

Whereas the extensive relations with Western Europe are largely informal, international callaborations with other nations are governed in part by formal bilateral agreements. The agreement with Japan appears to he very successful. The agreement with the People's Republic of China is working out well as they struggle to establish a world identity in high energy physics. Joint activities with the Soviet Union have decreased somewhat in recent years. The consensus remains that U.S.-Soviet exchanges are useful and should be maintained.

U.S.-Soviet Union cooperation in high energy physics is governed by the Bilateral Agreement on Cooperation in the Fundamental Properties of Matter, which operates under the Agreement on Cooperation in Atomic Energy, which was extended in 1983. Agreement on a yearly work program is determined by annual meetings of the Joint Coordinating Committee on Reseach on the Fundamental Properties of Matter (JCC/FPM). There have been some benefits under the JCC/FPM exchanges. More Soviet experimenters have participated in work at American acce1erators than have Americans at Soviet installations, but the Soviets have contributed novel instrumentation techniques that have been applied at American laboratories. Among these are the gas-jet target for 400-GeV experiments at Fermilab and the lithium high-current magnet lens for the Tevatron I antiproton source. A test facility for the electron cooling of proton beams, a technique invented in the Soviet Union at Novosibirsk, was designed and built at Fermilab a few years ago. These comprise significant "technology transfer" from the U.S.S.R. to the U.S.

Cooperation in high energy physics with Japan operates in part under a 10-year formal bilateral agreement initiated in 1978. It has consisted primarily of Japanese physicists working on U.S. programs, and equipment contributions, in exchange for access to U.S. facilities. With TRISTAN being brought into operation there is increasing interest on the part of U.S. physicists in doing experiments in this new facility. Currently an American-led group is assembling a major detector (AMY-CHAN) for use at one of the four TRISTAN intersection regions. There is also substantial U.S.-Japanese cooperation outside the formal agreement. 
Cooperation between the U.S. and People's Republic of China in. high energy physics is also governed in part by a bilateral agreement. Most of the collaborative efforts have been dominated by U.S. assistance in designing installations and instruments. The agreement is such that this assistance is without significant financial cost to the U.S. programs.

The $\mathrm{Kigh}$ Energy Commission under the aegis of the International Union of Pure and Applied Physics (IUPAP) has sponsored a regular cycle of international conferences in high energy physics, rotated among the Soviet Bloc, Western Europe, and the Unites States, and once in Japan. These have been eminently successful in furthering international communication, and in some cases have provided the first informal contact among scientists previously kept apart by political conditions.

Following the Economic Sumit Meetings at Versailles and Nilliamsburg, a Sumit Working Group on High Energy Physics.was set up to facilitate international cooperation in research and planning. The initial conclusions of the Working Group were the following:

i. Additional studies should be done on the possibility of expanded cooperation in accelerator technology development.

ii. The present intergovernmental working group should continue to meet periodically to ensure maximum collaboration over the coming years, regarding both existing machines and the planning of new ones.

iii. To benefit from international cooperation, both countries with major high energy physics programs and those with minor ones should have a strong home base program.

iv. If a largerinternational project is agreed upon, equity of benefits cannot be assured, but it can be approached through intensive negotiation on a case-by-case basis. Each participant should contribute in a way that leads to a balance of cooperation over the course of time.

The Working Group set up three new subpanels to facilitate the advance of high energy physics:

i. Subpanel for Long-Term Planning will receive from the Sumit nations their plans and proposals for high energy physics, with progress reports on existing major facilities, and will also take account of plans for high energy physics worldwide.

ii. Subpanel on Technical Collaboration will review activities tilat are underway or planned, to carry out the technical recomendations of the Subpanel on Improving International Cooperation in High Energy Physics

iii. Subpanel on Administrative Issues will review and make recommendations with respect to customs practices, data communications, and personnel exchanges. 
Meetings of the Working Group occurred in Washington in October 1983, in Brussels in July 1984 and in Cadarache, France, January 1985. The Group's activities are reviewed in "Annual Report to the Working Group on Technology, Growth, and Employment by the Sumit: Working Group on Bigh Energy Physics," April 1985, issued by the U.S. DOE.

Several conclusions on long-range planning were reached by the Working group. The present set of new high energy physics facilicies under construction, Tevatron and SLC in the U.S.; TRISTAN in Japan; LEP at CERN; and HERA in the Federal Republic of Germany, are complementary and not duplicative. A clear need not met by the present generation of accelerators is the requirement to extend the energy range of hadron colliders. The SSC is the most advanced plan in this regard and the Europeans are considering options in the LEP tunnel. Other open questions are a linear collider for electron positron collisions and an electron-proton collider which could also make use of the LEP tunnel. The required new and advanced facilities can be built and operated within broadly constant worldwide budgets, with some fluctuations during peak capital expenditure years. While further concentration of facilities is perhaps inevitable, more than one region with a forefront accelerator capability working effectively in high energy physics is essential. It is also essential that the limited number of unique facilities remain open to competent scientists from all over the world. Thus, $i=$ is considered of great importance to continue the discussion and planning on an intergovinnmental level.

With progress in high energy physics critically dependent on the availability of progressively more complex particle accelerators and versatile detectors, it has been necessary to develop new technology to ensure that these new facilities are achievable in the most economically feasible manner. Consequently, the technical cooperation subpanel recommended that the already active international collaboration in accelerator and detector technology be further encouraged; that coumon international standards be established to reduce costs and ensure compatibility; and that the existing high energy physics laboratories which are centers of advanced technology be used in extended ways for training in science and technology for industry, university, and other institutions.

It is clear that the removal of certain administrative obstacles would facilitate international collaboration via cost sharing and joint exploitation of regional facilities. Tariff and tax exemptions (e.g., on experimental equipment) are required for extended periods rather than the present short intervals. The freer exchange of scientific and technical staff requires simplified administrative country entry formalities, facilitated integration of the research worker's family in the host country, and a guarantee of adequate social coverage (e.g., health and accident insurance). Lastly, a productive international collaboration requires efficient international data transmission. To this end, it is necessary to review the changing policies for scientific data transmission across national borders and to promote effective data cormunication standards between nations.

In addition, the U.S. is actively pursuing opportunities for international cooperation in the Superconducting Super Collider (SSC) project, for example, through the Economic Sumit process. The U.S. 
representative and Chairman of the High Energy Physics Working Group has recently formally sent his Summit colleagues an open invitation for scientists from their countries to participate in the SSC effort. With the SSC being internationally available to collaborative experiments upon completion, it would be desirable to have some participation in the early $R$ and $D$ effort by scientists and engineers from those countries that are likely to be involved in the use of the facility. By such participation they would be better informed as to what international collaboration might best suit the future interests of their colleagues. In turn, the U.S. would benefit from their contribution to its $R$ and $D$ efforts to develop a plan for the best possible facility at the lowest possible cost.

Cooperation can take various forms extending from participation in the planning and design, to that of $R$ and $D$ on various problems, to the building of prototype systens, to construction, and to use. Early collaboration does involve the participation of capable and trained people where the contributions can be most significant and influential on the entire future success of the project. The summit offers an opportunity for greatly improving the international cooperation process.

\section{B. The European Program}

The European program in experimental high energy physics is centered at the European Organization for Nuclear Research Laboratory (CERN) in Geneva, Switzerland, and at the Deutches Electronen Synchrotron Laboratory (DESY) in lamburg, West Germany. CERN has 3600 staff members and an annual budget of $s 600 \mathrm{MSF}$; about $1800 \mathrm{high}$ energy physicists participate in the program. DESY has a staff of 1100 and an annual budget of $\curvearrowright 130 \mathrm{MDM}$. Although DESY is a national laboratory, it is utilized internationally, with groups from 11 nations and a total of 400 physicists participating in the program. These facilities are described below. For comparison, the 1984 worldwide budgets for high energy physics are shown in Table IX.

\section{CERN}

\section{a. Fixed-Target Operation of the Super Proton Synchrotron}

(SPS). The SPS is the mainstay of the experimental program at CERN. Its maximum energy has been upgraded to $450 \mathrm{GeV}$. The beam intensity achieved is now about $2 \times 10^{13}$ protons every 10 seconds. The West Experimental Area will be upgraded to provide two high energy, high quality secondary beams in addition to several test beams. New neutrino beams for beam dump experiments and for neutrino oscillation experiments are being constructed. The fixed-target program using the sPS is expected to remain strong during the second half of the $1980 \mathrm{~s}$.

b. The SPS Proton-Antiproton collider. This facility produces proton-antiproton collisions at a center-of-aass energy which has now reached $630 \mathrm{GeV}$ and with a peak luminosity of $4 \times 10^{29} \mathrm{~cm}^{-2} \mathrm{sec}^{-1}$ in each of two intersection regions. The integrated luminosity accumulated since 1981 is $0.6 \mathrm{pb}^{-1}$. The spps collider operates as follows: 26-GeV/C protons fron the PS are used to produce $3.5-\mathrm{GeV} / \mathrm{c}$ 
Table IX forld Budgets in High Energy Physics 1984

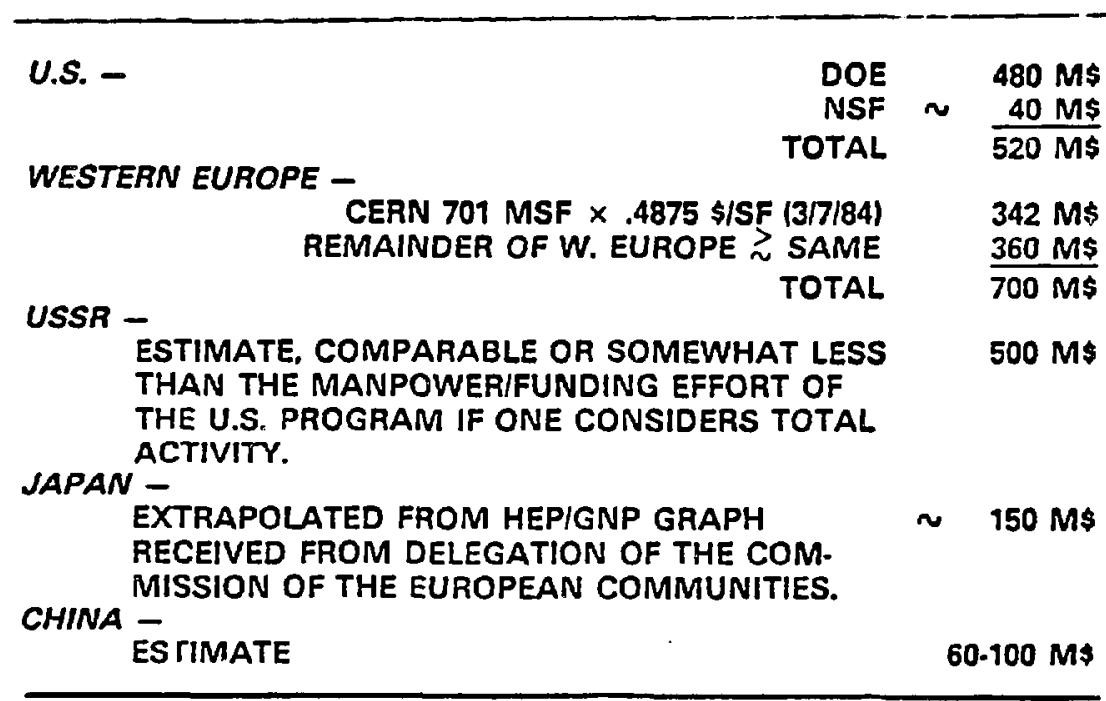

antiprotons, which are stored and stochastically cooled to form a dense beam in an accumulator ring (the AA). After enough antiprotons have accumslated and cooled, they are reinjected into the PS, accelerated to $26 \mathrm{GeV}$, and transferred to the SPS, where they are accelerated to $315 \mathrm{GeV}$ and caused to collide with counter-rotating bunches of $315-\mathrm{GeV}$ protons. The spectacular discoveries of the $\mathrm{W}$ and $2^{\circ}$ particles in 1983, and probably of the top quark in 1984, are all results of the first experiments with this facility. Improvements are underway to raise the luminosity to $3 \times 10^{30} \mathrm{~cm}^{-2} \mathrm{sec}^{-1}$ by 1987 , with an added $3-\mathrm{GeV}$ ring to enhance the accumulation of antiprotons (ACOL), (leading to an experced integrated luminosity of $10 \mathrm{pb}^{-1}$ during 1988 ). and to upgrade the UA1 and UA2 detectors.

The UA-1 detector is being upgraded by improving the calorimeter energy resolution, by adding a high resolution vertex detector, and by extending the muon detection system to a larger solid angle. In addition to these detector improvements, the data acquisition system will be upgraded to match the increase in luminosity. The muon detection system improvements will be completed in 1985, and the remainder of the improvements will be completed in 1987. These improvements will lead to better energy resolution for electrons, muons, and jets and will, thus, enhance the detectors' ability to determine the transverse energy carried away by particles which do not interact in the detector, such as neutrinos. With 23 U.S.-supported physicists among the $178 \mathrm{UA}-1$ collaborators, this detector represents a significant effort for the U.S. as well as for Europe.

The UA-2 detector will be upgraded by extending the hadron calorimeter coverage from $40^{\circ}$ to $5^{\circ}$ with respect to the beam axis. This will be accomplished by replacing the toroidal magnets with calorimeters, thereby making the detector a totally non-magnetic detector. Other changes will be made to improve the electron identification and tracking in the central calorimeter. 
Table $\mathrm{X}$ Comparison of the Sp̄pS and Tevatron Colliders

\begin{tabular}{|c|c|c|c|}
\hline & $\begin{array}{c}\operatorname{spps} \\
\text { (upgraded/1988) }\end{array}$ & $\begin{array}{l}\text { Tevatron I } \\
\text { (1987) }\end{array}$ & $\begin{array}{c}\text { Tevatron I } \\
\text { (upyraded/1989) }\end{array}$ \\
\hline Center-of-viass Energy (GeV) & 630 & 1800 & 2000 \\
\hline Peak Luminosity $\left(\mathrm{cm}^{-2} \mathrm{sec}^{-1}\right)$ & $3 \times 10^{30}$ & $10^{30}$ & $10^{31}$ \\
\hline Events of $W \pm e^{ \pm}=$ & $1.0 \times 10^{4}$ & $1.2 \times 10^{4}$ & $1.4 \times 10^{5}$ \\
\hline Events of $Z^{\circ} \rightarrow e^{+} e^{-}$ & $3.6 \times 10^{2}$ & $9.0 \times 10^{2}$ & $1.1 \times 10^{4}$ \\
\hline Number of $t$ and $\bar{t}(40 \mathrm{GeV} / \mathrm{c})$ & $6.0 \times 10^{4}$ & $1.8 \times 10^{5}$ & $2.2 \times 10^{6}$ \\
\hline $\begin{array}{l}\text { Mass Limits for Heavy } y \\
\left(\text { GeV/C } C^{2}\right)^{\star \star}\end{array}$ & 240 & 500 & 700 \\
\hline $\begin{array}{l}\text { Mass Limits for Heavy } Q \\
\left(G=V / c^{2}\right)^{\star \star}\end{array}$ & 90 & 130 & 220 \\
\hline
\end{tabular}

T Tine yields assume a run of $10^{\prime}$ seconds at peak luminosity.

* ine limits are based on the production of 100 produced particles in a run of $10^{7}$ seconds at peak luminosity.

A relative comparison of the discovery potential of the Sp̄pS and the Tevatron can be made by comparing the production of the standard electroweak bosons, more massive gauge bosons ( $V$ ), and heavy quarks (Q) in a run of $10^{7}$ effective seconds. The results are given in Table $X$, which considers planned luminosity improvement programs at both Colliders.

c. Large Electron-Positron Facility (LEP). LEP is an electron-positron storage ring with a circumference of $27 \mathrm{~km}$ and eight straight sections. Phase 1 of LEP, aimed at providing $60 \mathrm{GeV} \times 60 \mathrm{GeV}$ electron-positron collisions in four intersection regions was approved by the CERN Council at its October 1981 meeting. The predicted peak luminosity is $10^{31} \mathrm{~cm}^{-2} \mathrm{sec}^{-1}$. The. CERN schedule calls for the first colliding beams by the start of 1989. In Phase 1 , only $1 / 6$ of the available rpace along the ring will be equipped with conventional radiofrequency accelerating cavities. With the full complement of conventional cavities, LEP will be able to reach $85 \mathrm{GeV} \times 85 \mathrm{GeV}$. If the superconducting accelerating cavities are developed successfully and employed in LEP, an energy upgrade of LEP as high as $120 \mathrm{GeV} \times 120 \mathrm{GeV}$ will be possible. A capsule of the project is shown in Table XI. The four planned detectors for LEP (ALEPH, DELPHI, OPAL, and L3) are composed with those for SIC (MARK II and SLD) in Section II.B.2.d.

At center-of-mass energies of about $200 \mathrm{GeV}$, LEP will permit searches for new types of leptons and quarks up to masses of $100 \mathrm{GeV}$. It will permit study of the production of $\mathrm{z}^{\circ} \mathrm{z}^{\circ}$ pairs and $\mathrm{w}^{+} \mathrm{w}^{-}$pairs 
Taole XI LEF Project

- $\theta^{+}$- StORAGe RING - 60 - 120 GeVIBEAM

-120 - 240 GeV CENTER-OF-MASS

- CIRCUMFERENCE - $27 \mathrm{~km}$ (17 mi)

- location - eURopean CENTER for paAticle physics RESEARCH, CERN, GENEVA, SWITZERLAND

- AUTHORIZED FOR CONSTRUCTION - DECEMBER 1981

- COST - 910 iASF (1981). EXCLUDING LABOR

- ESTIMATE 2000 MSF WITH LABOR

- SCHEDULE - INITIAL OPERATION: START OF 1989

- DETECTORS - 4 OF 6 INTERACTION REgIONS INITIALLY

and searches for heavier vector bosons and other particles. LEP will also be able to search for Higgs particles up to a mass of $70 \mathrm{GeV}$. These experiments address some of the most fundamental questions with respect to our understanding of electroweak processes, including the origin of symmetry breaking and the origin of masses.

d. The Next Step at CERN. The European high energy physics community is considering the installation in the LEP tunnel in the mid1990 s of a superconducting magnet ring suitable for storing 5-and perhaps up to $10-\mathrm{TeV}$ protons. This has already been the subject of several European workshops and was discussed at the 1984 meeting in Japan of the International Committee on Future Accelerators (ICFA). Such a facility, designated the large hadron collider (LHC), would probe near the $1-\mathrm{TeV}$ mass range but with capabilities significantly lower than those of the SSC facility being considered in the U.S. A decision may be reached in 1987 .

\section{DESY}

a. DORIS. The electron-positron collider ring DORIS has been rebuilt with more accelerating capability added to raise the maximum reaction energy to $12.0 \mathrm{GeV}$. Both interaction regions are equipped with mini-beta beam-focusing optics to allow high luminosity operation ( $\backsim 300$ to $400 \mathrm{nb}^{-1}$ per lday). DORIS is operating with two complementary detectors: a large solenoid detector, ARGUS, and the Crystal Ball detector relocated from SPEAR. These will be used in a detailed study of bound bottom quark states and of decays of particles containing bottom quarks.

b. PETRA. The electron-positron collifing ring PETRA has so far operated at reaction energies between 10 ind $45 \mathrm{GeV}$. The installation of a mini-beta beam-focusing system in all four interacting regions has resulted in a peak luminosity above $10^{31} \mathrm{~cm}^{-2}$ $\mathrm{sec}^{-1}$ and an average integrated luminosity between 400 and $600 \mathrm{nb}^{-1}$ per day per interaction region. A program has been implemented 
recently to increase the maximum reaction energy to about $45 \mathrm{GeV}$ in two steps. An increase from 36.7 to $40.5 \mathrm{GeV}$ was achieved with a doubling of the if power from 4 to $8 \mathrm{MW}$. With the installation of rf cavities in the two remaining long straight sections the energy was increased from 40.5 to about $45 \mathrm{GeV}$ in early 1983. (Actually, a peak energy of $47.6 \mathrm{GeV}$ has been reached since that time.) Superconducting If cavities would be required to increase the energy beyond $45 \mathrm{GeV}$ up to a maximum of 60 to $70 \mathrm{GeV}$. A superconducting cavity built by Karlsruhe was successfully tested with high rf power and was installed at PETRA in 1982. DESY, in collaboration with CERN and Wuppertal, has a program to build and test superconducting cavities, but it is unlikely that such an energy upgrade for PETRA will be pursued.

c. HERA. The Deutsches Elektronen-Synchrotron (DESY) has begun the construction of a large electron-proton colliding beam facility, HERA, designed to collide either electrons or positrons of $30-\mathrm{GeV}$ nominal energy with protons of energies up to $820 \mathrm{GeV}$, yielding $314-\mathrm{GeV}$ reaction energies and a maximum momentum-transfer-squared, $Q^{2}$, of 98,000 $\mathrm{GeV}^{2}$. This is equivalent to an electron beam of $52 \mathrm{TeV}$ on a stationary target. The design luminosity of $2.5 \times 10^{31} \mathrm{~cm}^{-2} \mathrm{sec}^{-1}$ will allow the determination of the proton structure function in a substantially new range of momentum transfers, $2000(\mathrm{GeV} / \mathrm{c})^{2}<\mathrm{Q}^{2}<10000(\mathrm{GeV} / \mathrm{c})^{2}$, far beyond anything acheivable anywhere else in the world. (The maximum $Q^{2}$ achievable in deeply inelastic muon or neutrino scattering at the Tevatron II is about $\left.400(\mathrm{GeV} / \mathrm{c})^{2}\right)$. These experiments, together with observations of the final state, will allow new tests of the Standard Model, especially of perturbative QCD. The electron and positron beams are expected to be transversely polarized, and DESY has plans to enable rotation of the polarization, to provide longitudinally polarized electrons and positrons, an extremely useful capability.

Construction of HERA began in the spring of 1984, with initial operation scheduled for the last half of 1989. A capsule of the project is shown in Table XII.

\section{The Japanese Program}

Elementary particle research in Japan has so $f$ ar been carried out at the $12-\mathrm{GeV}$ proton synchrotron at KER. The future program involves the construction at KER of a large storage ring complex, TR.ISTAN, designed to collide electrons and positrons and, perhaps at a later stage, electrons (or positrons) wi th protons. The TRISTAN tunnel will have a total circumference of $3 \mathrm{~km}$ with four interaction regions. An electron-positron colliding ring capable of exploring reaction energies up to $60 \mathrm{GeV}$ will be housed in this tunnel. TRISTAN covers an energy range not covered by any other existing or planned $e^{+} e^{-}$collider and thus provides an important new opportunity to discover and study any new phenomena whose thresholds lie in this range. Its physics program will probably stress electroweak interference effects, jet formation and fragmentation, and studies of the toponium system if $\bar{t} t$ bound states are within its energy range. A maximun luminosity in the range ( 3 to 8 ) $\times 10^{31} \mathrm{~cm}^{-2} \mathrm{sec}^{-1}$ is expected. 
Table XII EERA Froject

- ep storage Rings - $820 \mathrm{GeV}$ p COLLIDING WITH $30 \mathrm{GeV}$ e - 314 GoV CENTER-OF-MASS

- CIRCUMFERENCE - $6.3 \mathrm{~km}(4 \mathrm{mi})$

- LOCATION - DEUTSCHES ELEKTRONEN SYNCHROTRON (DESY), HAMBURG, WEST GERMANY

- AUTHORIZED FOR CONSTRUCTION - APRIL 1984

- COST - 950 MDM (1984). EXCLUDING LABOR

- ESTIMATE 2000 MDM WITH LABOR

- SCHEDULE - INITIAL OPERATION: LAST HALF OF 1989

- Detectors - INITIAL COMPLEMENT of 3 Detectors

PLANNED. LETTERS OF INTENT TO BE SUBMITTED

BY JUNE 30, 1985.

- collaborating nations - Canada. france, isRael italy. NETHERLANDS. AND UNITED KINGDOM

There are three major detectors at TRISTAN; two of them, TOPAZ and VENUS, are general purpose detectors. VENUS uses a drift chamber in a solenoidal magnetic field for charged particle tracking and a lead glass array for photon detection. TOPAZ also features lead glass but uses a TPC for charged particle identification and tracking. Both detectors are being constructed by Japanese collaborations. The third detector, AMY, is a compact detector emphasizing lepton and photon detection. To this end, the detector uses a high magnetic field, high resolution tracking chambers, and a photon counter before the magnet coil. This detector has major U.S. collaboration and leadership.

The TRISTAN project has been authorized, and construction is underway. The start of the experimental program is scheduled for late 1986. The installation of superconducting rf could bring the energy to $36 \mathrm{GeV}$ per beam.

D. The U.S.S.R. Program

1. Institute for High Energy Physics (IHEP), Serpukhov

The major proton accelerator facility in the U.S.S.R. at present is the 76-GeV synchrotron at IHEP. Although its proton beam intensity is low by Fermilab and CERN SPS stantards, it supports an active program, similar to that at the AGS at BNL, with emphasis on large, relatively permanent experimental setups. Some unique work on cham and particle production by hadrons is being done in a neutral beam; a neutrino counter experiment has ceased operation until the new booster comes into operation to increase the intensity. Work on $\mathrm{K}^{\circ}$ regeneration experiments has been in progress for 10 years; a large multiparticle spectrometer facility has been used to study charmed particles. A group using emulsion techniques has provided the cold emulsions for the search for charmed particles in the Fermilab neutrino beam using the 15-ft Ferailab bubble chamber. SKAT, the 
large propane chamber for neutrino physics, is still in operation as is MIRABELLE, a large bubble chamber for hadron physics. The bubble chamber scanning and measuring facilities at both Dubna and Serpukhov are extensive and have recently been upgraded.

In the future the $76-\mathrm{GeV}$ synchrotron will become th 3 injector for a multi-TeV accelerator/storage ring, UNR. Phase I of UNK, which was authorized for construction in 1981 , includes a $400-\mathrm{GeV}$ booster synchrotron using conventional magnet technology and a $3000-\mathrm{GeV}$ ( $3-\mathrm{TeV})$ synchrotron using superconducting magnets whose design is similar to Fermilab's. Phase I will provide fixed-target beams to 3000$\mathrm{GeV}$ maximum incident energy ( $75-\mathrm{GeV}$ reaction energy) and proton-proton colliding beams of $400 \times 3000 \mathrm{GeV}$ ( $2190-\mathrm{GeV}$ reaction energy): $\overline{\mathrm{p} p}$ collisions at $2.2-\mathrm{TeV}$ center-of-mass energy would be possible at this stage. Phase II, which is still in the planning stage, could provide $3000 \times 3000-\mathrm{GeV}$ proton-antiproton colliding beams and, with the addition of a second superconducting magnet ring, $3000 \times 3000-G e V$ protonproton collisions. If both phases of UNR are successfully completed and operated, the U.S.S.R. will have a high energy physics research capability in the 1990s far beyond that provided by any facility currently authorized. However, either the SSC in the U.S. or the LHC at CERN would surpass the capabilities envisioned for UNK.

\section{Institute for Nuclear Physics (INP), Novosibirsk}

This Institute is the center of lepton interaction physics in the Soviet Union. The research centers around the VEPP- 4 electronpositron storage ring, which has a maximum energy of $5.8 \mathrm{GeV}$ per beam. A Iuminosity of $3 \times 10^{30} \mathrm{~cm}^{-2} \mathrm{sec}^{-1}$ has been obtained at $5 \mathrm{GeVx} 5 \mathrm{GeV}$ (10$\mathrm{GeV}$ reaction energy). This device has been used to make precision measurements of masses of particles containing the charmed quark. An older storage ring, VEPP-3, is used as an accumulator/injector for VEPP-4, allowing the electrons and positrons to polarize before injection. Pclarized beams of $>50 \%$ have been achieved. The main detector facility employs a 400-ton magnet with field perpendicular to the plane of the particle orbits. While the physics research program at INP is considered modest by Western standards, the contributions of INP to the development of accelerator technology have been exceptiona1. INP is reported to be involved with design of an antiproton source for use with UNK. This effort stems from the pioneering work at INP on electron cooling of proton beams.

\section{Joint Institute for Nuclear Research (JINR), Dubna}

Although the accelerator facility at Dubna, the $10-\mathrm{GeV}$ synchrophasotron is not competitive by modern standards, its high energy physics program remains active. Its group is allocated about $30 \%$ of the research time at Serpukhov and maintains a team of physicists on-site to support this research. At: Dubna, work is being done on quark models of the nucleus, using heavy ion beams, as well as some fine polarized jet target research using a very advanced and effective solid state detector array. 
E. The Program of the People's Republic of China

The major high energy physics facility under construction in the People's Republic of China is a $2.5 \mathrm{GeV} \times 2.5 \mathrm{GeV}$ electron-positron collider designated BEPC. It is designed to achieve high luminosity to support an experimental program focused on high precision studies of the particle states containing charmed quarks. The design luminosity is $1.7 \times 10^{31} \mathrm{~cm}^{-2} \mathrm{sec}^{-1}$ with one bunch per beam. Initial operation is expected in 1988 .

IV. NEW FACILITY NEEDS FOR THE U.S. HIGH ENERGY PHYSICS PROGRAM

\section{A. The Next Step}

In the past, major scientific breakthroughs in high energy physics have been associated primarily with investigations at higher and higher energies. Recent insights have led to the conclusion that exploration of the $\mathrm{TeV}$ mass scale (or equivalently of the $10^{-17} \mathrm{~cm}$ distance scale) is the next step toward any major advance.

This could be accomplished with a Superconducting Super Collider (SSC), a device that collides head-on two beams of protons, each of energy $20 \mathrm{TeV}$. The proposed SSC (Figure 22) would consist of an injector complex of accelerators, of approximately the scale of the Fermilab Tevatron facility, to accelerate protons from rest to an energy of $\mathrm{I} \mathrm{TeV}$ or more for injection into two very large rings of superconducting magnets that confine the counter-rotating bunches of protons in the desired orbits. These bunches of high energy protons are then accelerated to an energy of $20 \mathrm{TeV}$ and caused to collide at six symetrically placed positions around the rings where facilities for physics experiments can be located. The circumference of the main ring is determined by the strength of the main ring dipole magnets which bend the trajectory of the protons. For the range of dipole magnetic fields considered at this time, the circumference of the main ring is between 90 and $164 \mathrm{~km}$. Figure 23 shows the size of a $90-\mathrm{km}$ SSC main ring compared with the 6-km-circumference Tevatron and the 27-km-circumference LEP facility.

The energy of the SSC and its high design luminosity $\left(10^{33} \mathrm{~cm}^{-2}\right.$ $\mathrm{sec}^{-1}$ ) represent a substantial extension of the major parameters of colliders, proposed or under construction. The SSC will be able to probe to a much higher mass scale or to smaller subnuclear distances than are accessible with any other existing or planned facility. It thus offers a real probability of major advances in our knowledge c $f$ elementary particles and the unified forces of nature. Such a device is now within reach of present-day technology because of the success of U.S. investment in superconducting magnet technology and the investment of the world HEP laboratories in high energy beam technology. The SSC could be operational in the mid 1990 s.

The 20-TeV proton-proton collider envisioned for the SSC have been discussed for some time. Two workshops sponsored by the International Committee for Future Accelerators (ICFA), at Fermilab in 1978 and at CERN in 1979, considered the technology of such accelerators and other potential frontier instruments for reaching very high 

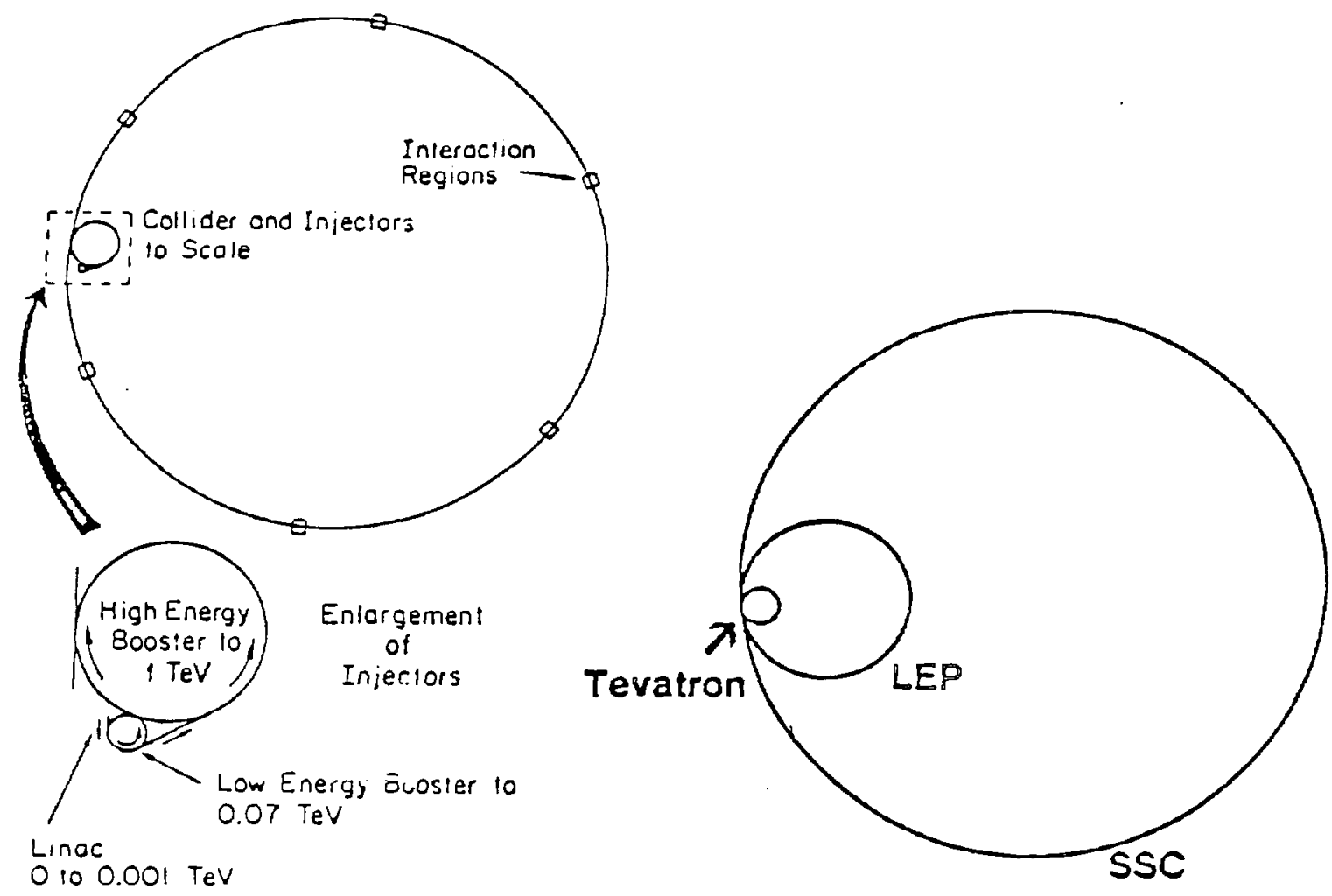

Figure 22 (Left). The layout of the SSC used in the Reference Designs Study, indicating the injector complex and the main ring where protons are accelerated to $20 \mathrm{TeV}$ in counterrotating bunches that collide at six points along the circumference.

Figure 23 (Right). The relative sizes of the Tevatron at Fermilab (6-km circumference), the LEP collider under construstion at CERN (27-km aircumference), and the SSC main ring (90-km circumference if constructed with $6.5-\mathrm{T}$ bending magnets).

energies but left costs and engineering details to the future. Since then, magnet and colliding beam technologies germane to proton-proton colliders have made substantial advances.

These technical advances and recognition of the enormous scientific potential of a multi-TeV proton-proton collider led U.S. leaders in high energy physics to suggest construction of such a collider as soon as possible. Figure 24 indicates some of the physics motivation for the SSC.

The U.S. high energy physics community started to consider this question at the 1982 Summer Study of the American Physical Society (APS) Division of Particles and Fields at Snowmass, CO (June 28 to July 16). The purposes of the Summer Study were to assess the future of high energy physics, to explore the limits of our technological capabilities, and to consider the nature of future major facilities in the U.S. The 1982 Sumer Study led to a widespread recognition of the need for (and importance of) a multi-TEV proton-proton collider, based 


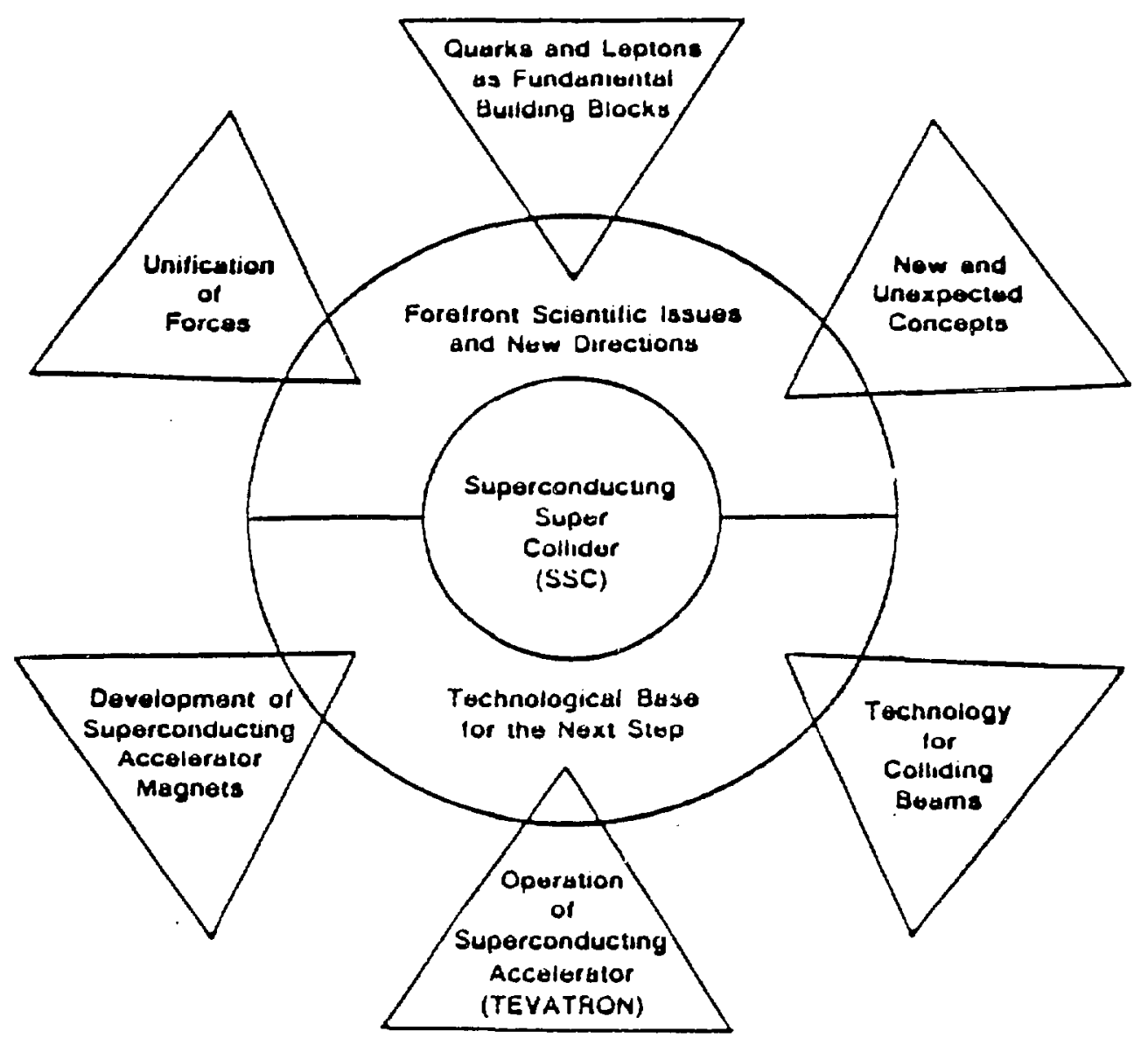

Figure 24. In recent years progress in theoretical and experimental physics has pointed to several forefront scientific issues and new directions for research that must be addressed by a vital U.S. high energy physics program, and technical developments have provided a strong base for the next step in major facility development and construction. The SSC concept represents the response of the U.S. high energy physics commity to these factors and opportunities.

in part on recent accomplishments (in both experimental and theoretical research) strongly suggesting that the next major step will require exploration of the energy region where new particles (with new properties and masses of a few $\mathrm{TeV}$ ) can be produced.

At a 1983 20-T $3 \mathrm{~V}$ Hadron Collider Technical Workshop at CornelI University (March 28 to April 20), 40 U.S. and European experts in accelerator science and accelerator construction technology met to consider further the technical issues. They concluded that the time was ripe to intensify development of candidate magnet systems in order. to allow design and cost studies that would narrow cost uncertainties and define construction methodologies more sharply. Assuming a reasonable level of effort, they thought construction could begin within four years.

The APS Division of Particles and Fields sponsored a 1983 Workshop on Hadron Collider Detectors: Present Capabilities and Future 
Possibilities, at Lawsence Berkeley Laboratory (February 28 to March 4), attended by about 100 experimenters, mostly from the U.S. The issues of detector technologies in the face of the multiplicities expected from machines like the SSC were addressed, and the general conclusion was that, although difficult, the required experiments will be possible.

In February 1983, a HEPAP Subpanel on New Facilities was formed to make recomendations relative to the scientific requirements and opportunities for a forefront U.S. high energy physics program in the next five to ten years. Following a series of on-site meetings at the three DOE accelerator centers, the Subpanel completed its report in two lengthy deliberative meetings in June-July 1983. Its first and unanimous -ecommendation was for the immediate initiation of a project aimed at the design and construction of a multi-TeV, high-luminosity proton-proton collider, designated the Superconducting Super Collider (SSC). This recommendation was unanimously endorsed by HEPAP with the highest priority. In his letter transmitting the Subpanel report, the Chairman of HEPAP commented that the SSC "... has fired the imagination of high energy physicists everywhere. The SSC would be the forefront high energy facility of the world and is essential for a strong and highly creative United States high energy physics program into the next century."

After reviewing the HEPAP recommendation, the DOE decided to initiate $R$ and $D$ for the SSC. The DOE then used its advisory mechanism to provide input on the direction of advanced accelerator $R$ and $D$, preliminary to a proposal for constructing the SSC. As requested in August 1983 by the Director of the DOE Office of Energy Research, HEPAP set up a subpanel to provide advice and recommendations on the content and :uplementation of an FY $1984 \mathrm{R}$ and $\mathrm{D}$ effort, preliminary to the initiation of a formal SSC R and D program. In the fall of 1983, the subpanel reviewed the various requests to carry out relevant $R$ and $D$ and recommended several different approaches to SSC magnet design, including high field (8- to 10-tesla) magnets based on $\mathrm{Nb}_{3} \mathrm{Sn}$ superconductor, medium field (5- to 6-tesla) magnets based on $\mathrm{Nb}-\mathrm{Ti}$ superconductor, and low field (2- to 3-tesla) superferric magnets. It also recommended support for development of $\mathrm{Nb}_{3} \mathrm{Sn}$ conductor, accelerator physics activities, and other critical technical $R$ and $D$ efforts. The subpanel's recomendations were endorsed by HEPAP and transmitted to the DOE Office of Energy Research.

In addition to the work by HEPAP, other activities have provided significant input from the high energy physics and accelerator physics communities (as well as the relevant technical and industrial communities) into the nationwide consideration of the scope, design, and use of the SSC and of the $R$ and $D$ required to generate an optimized design. These activities include the following:

i. Ad hoc Physics at the SSC (PSSC) Meetings of the potential SSC user community, held periodically since 1983 at various universities and laboratories, attended by about 200 U.S. physicists, focus on the required scientific capability for the SSC as best determined from recent theoretical and experimental results, and on the anticipated requirements for detector instrumentation. 
ii. The 1983 APS Division of Particles and Fields Workshop on Accelerator Issues for the SSC at the University of Michigan, Ann Arbor (December 12-17) was attended by 70 accelerator specialists from many laboratories and universities. Its goals were to identify the accelerator physics issues crucial to demonstrating the feasibility of the SSC and the key $R$ and $D$ needs, and to guide the engineering design of technical components and systems.

iii. The 1984 APS Division of Particles and Fields Cryogenic Workshop at Brookhaven National Laboratory (January 17-19) was attended by about 100 experts in cryogenic systems, superconducting magnet design and fabrication, and accelerator physics, who addressed the cryogenics issues most pertinent to the design of a large-scale accelerator using superconducting magnets.

iv. Implementation was completed by DOE, in early 1984, of an interim management arrangement to develop $R$ and $D$ and management $p l a n s$ for a preconstruction $R$ and $D$ and proposal development phase of the SSC activity. These plans were submitted to DOE in July 1984.

v. The 1984 APS Division of Particles and Fields followup workshop to the 1982 Snowmass workshop, at Snowmass (June 23 to July 13), reaffirmed the SSC as the facility essential to ensure a vigorous and creative U.S. program in high energy physics in the latter part of this century and the early part of the next.

In December 1983 the directors of the U.S. high energy accelerator laboratories with DOE concurrence chartered the National SSC Reference Designs Study to review in detail the technical and economic feasibility of various options for creating the Superconducting Super Collider (SSC) facility as a $20-\mathrm{TeV}$ on $20-\mathrm{TeV}$ proton-proton collider having a luminosity up to $10^{33} \mathrm{~cm}^{-2} \mathrm{sec}^{-1}$. The objective was to assess the technical feasibility, develop a cost estimate based on clearly stated and credible assumptions, and to help define how best to proceed wi th SSC $R$ and $D$ directed toward improving the cost effectiveness of applicable accelerator technology. The study was based on three kinds of super-conducting magnets, with different configurations, and was aimed at sharply decreasing the cost of ' needed magnet system below that of existing designs. It addressed three key areas: technical feasibility, economic feasibility, and identification of specific $R$ and $D$ needs, with primary emphasis on the range within which the SSC construction cost -an confidently be expected to fall--excluding the cost of research equipment, preconstruction $R$ and $D$, and site acquisition. The results indicated that the basic design principles used successfully for existing accelerators can be conservatively extended to a proton collider having the SSC primary specifications of energy and luminosity, and that each of the three reference magnets could serve as the foundation for such a collider. Vigorous $R$ and $D$ would be needed to refine the cost estimates for the magnets, to determine their actual performance, reliability, and manufacturability, and to develop cost-effective methods for their assembly and quality assurance. A6.5-tesla magnet was selected in 1985 as part the $R$ and $D$ program. An important goal will be to produce a significant number of magnets by mass-production 
methods, and to test them under conditions simulating actual accelerator operation.

The estimated construction costs for an SSC facility based on the three $k$ inds of magnets, range from $\$ 2.70$ to $\$ 3.05$ billion (FY 1984 dollars). These include sufficiently conservative contingencies that they represent the current best estimate for an upper bound on the SSC cost.

A DOE comittee, including consultants, reviewed the National SSC Reference Designs Study Report in May 1984, and concluded that the Report establishes the technical feasibility, economic credibility, and specific $R$ and $D$ needs of the proposed SSC project. The comittee recommended increasing the total estimated construction cost by 0.2 billion dollars, and concluded, on the basis of an analysis of the sensitivity of the cost estimate to various critical assumptions, that an upper limit on the SSC cost is no more than 1.25 times the cost of the most expensive design considered in the Study. The committee judged the proposed six-jear construction schedule to be feasible.

A decision to proceed with the preconstruction $R$ and $D$ and proposal development phase of the SSC effort was made by the DOE during the summer of 1984 .

In preparation for the SSC, research and development are continuing in FY 1985. A critical milestone of the 1985 program is selection of the magnet type. The efforts in FY 1985 were concentrated on magnet $R$ and $D$ to provide a technical basis for this decision, but they also included work on a siting parameter document. In FY 1986 $K$ and $D$ is expected to focus on a cost-optimized design of the selected magnet, conceptual designs of conventional and technical systems (including cost and schedule estimates), and fabrication of magnet prototypes in preparation for spring tests in 1987.

B. Accelerator $R$ and $D$ in Superconductivity

For large circular colliders, it is imperative to use superconductivity to keep costs down, and to keep power consumption and/ or size within reasonable limits.

In the area of hadron colliders, substantial $R$ and $D$ on superconducting magnets is required to continue the improvement in performance, to lower even further the total cost of such colliders, and to increase magnet reliability. This $R$ and $D$ work (being performed in the U.S. at BNL, Fermilab, LBL, and the Texas Accelerator Center) can conveniently be subdivided into two categories:

1. Development of new conductors and related magnet construction techniques suitable for fields $\geq 8 \mathrm{~T}$ (high fields).

2. Improvement of conductors and techniques for fields in the range $2 \mathrm{~T}$ to $6 \mathrm{~T}$ ( 1 ow to mediurn fields).

The most substantial basic development is required for the first category, wile cost considerations are important for both categories in order to allow the final choice to be made on sound economic grounds. Two lines of development are open in the area of high-field con-

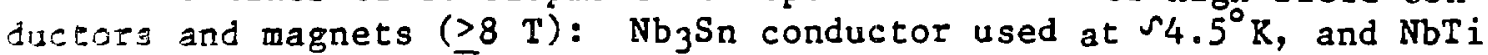


used at $\checkmark 2^{\circ} \mathrm{K}$. In principle the most promising material is $\mathrm{Nb}_{3} \mathrm{Sn}$ because of its higher critical field and higher temperature (more relaxed cryogenic system). Its drawback is brittleness and fragility, which requires either final reaction of the composite to obtain the superconducting state after winding, or else magnet coils wound with relatively large radii using pre-reacted matexial. $\mathrm{Nb}_{3} \mathrm{Sn}$ has been produced for many years, but the specific requirements for this application of small-bore magnets operating over a wide field range (maximum field about 20 times the injection field) are high current density and superconducting filaments of small diameter. Winding and insulation techniques compatible with a heat treatment of $\checkmark 700^{\circ} \mathrm{C}$ for a few hours, or methods of avoiding too small bending radii in winding of pre-reacted material, must also be developed.

The alternative line of development toward high fields is to use NbTi conductors at lower temperature $\left(\wedge 2^{\circ} \mathrm{K}\right)$. The advantage is that the material can be wound in the reacted state by means of well established techniques; the disadvantages relate to the cryogenic system - more complicated cryostats and larger power consumption. The maximun field is also more limited. This line is a convenient reserve should the development of $\mathrm{Nb}_{3} \mathrm{Sn}$ encounter serious difficulties or lead to excessive costs.

Work based on medium-field magnets $(4.5$ to $6 \mathrm{~T}$ ) is a natural contindation of present work. The $R$ and $D$ is directed toward low cost and the development of industrial manufacturing techiques. The conductor development would be essentially the same as that required for the high-field case with $\mathrm{NbTi}$ at $2^{\circ} \mathrm{K}$.

Still lower fields, between 2 and $3 \mathrm{~T}$, are also being considered. In this case the field distribution is shaped by iron boundaries, and the role of superconductor is to minimize the power consumption. These magnets are called superferric. The development needed here is directed toward simple, inexpensive design and costeffective manufacturing techniques in order to counterbalance the increase in cost attributable to the longer tunnel and more spread-out infrastructure that would be required.

International collaboration in these superconducting magnet development programs would be very beneficial. Such work should be aimed at enhancing technology transfer to the industries of the collaborating regions. Specific elements in such a collaboration might be the following:

- Common definition of possible new superconductors in order to minimize industrial investment for development and to enlarge potential markets.

- Joint selection of a small number of potentially interesting techniques and conceptual magnet designs to be tested by means of models.

- Eventually, coordinated fabrication and evaluation of fullscale prototypes.

To assess the possibility of acheiving these goals, the exchange of people between interested laboratories should be encouraged and supported. 
It should be emphasized that all of these developments could be beneficial to a number of applications in other fields such as fusion, electrical power transmission, cryogenerators, energy storage and recovery, magnetic separation of minerals, nuclear magnetic resonance for medical and other applications, transportation, etc.

The large superconducting magnet systems described above require very substantial cryogenic systems, distributed over long distances, for the production, transfer, and recovery of He. Simple cryostats, a reliable and efficient liquifier plant, and low-loss transfer lines are very important elements of a satisfactory design, which must be tailored to each specific accelerator project. One point that deserves a careful assessment by experts is the possibility of using superfluid the at $s 2^{\circ} \mathrm{K}$ for such applications. An evaluation of the additional costs with respect to normal $4.5^{\circ} \mathrm{K}$ systems, because of their increased cryostat complexity and power consumption, is a necessary first step.

During the last few years superconducting radiofrequency ( $r f$ ) accelerating structures have matured to the point where their use in large circular accelerators seems feasible and realistic. Multicell niobium structures have reached average accelerating gradients of more than $4 \mathrm{MV} / \mathrm{m}$ at negligible rf-power loss. Even considering the fact that these small remaining losses occur at the temperature of liquid helium and require powerful refrigerators, the overall power economy of superconducting resonators is one to two orders of magnitude better than that of conventional copper structures. The fact that the accelerating gradients are more than a factor of four higher than those in copper structures (in $\mathrm{cw}$ operation) makes these superconducting resonators very well suited to applications in which large rf accelerating voltages are required in a continuous operation, i.e., in electron and proton storage rings.

These new structures have been studied at a number of different laboratories in the U.S. Germany, France, Japan, Italy, and at the CERN laboratory. Successful and reliable operation at high accelerating gradients has been demonstrated at the electron-positron storage rings CESR (Cornell University) and PETRA (DESY, Hamburg). To make this new technology more economical and attractive for routine operational use, vigorous development programs are now under way at several laboratories. These efforts include the development of copper structures with a superconductive niobium coating, simplifications in the cryogenic technology, special cavity shapes to suppress excitation of higher resonances, and simple techniques for industrial production.

\section{Longer Range}

For the longer range, beyond 1995 , several possibilities are envisioned for new or expanded facilities to allow continued vitality and U.S. leadership in high energy physics. These could include upgrading the SSC, a TeV-range colliding 1 inear accelerator, or some type of facility not yet imaginable.

The concept of colliding beams with linear accelerators as a possible approach to high energies in the next century has been discussed within the high energy physics community and informally with the 1983 
HEPAP Subpanel on New Facilities for the U.S. High Energy Physics Program. In this concept two opposing, collinear linear accelerators would accelerate a series of charged bunches toward each other in such a way that collisions between the bunches take place in the space between the linacs, where sophisticated beam optical systems focus the beams to submicron size at multiple collision points displaced laterally from one another. Different bunches collide at different collision points.

Technical success with such a colliding beam system depends on the ability to produce and maintain high phase-space density bunches in the face of strong space-charge and wakefield forces, and on the ability to bring these bunches to a sharp, steady focus of typical dimension 0.1 micron: 10 times smaller than that sought in the SLC. In addition, luminosity multiplication through self-pinching of the opposing beam bunches during collision is crucial for achieving the desired luminosity. Some confidence about understanding these factors will be gained in the course of bringing the SLC into operation, but the order-of-magnitude reduction in beam size and transverse phasespace area for the multi-TeV case should be emphasized.

Economic success with this direct evolutionary approach depends on considerable lowering of unit costs, both capital and operating. Major items in the capital cost are the rf sources, including klystrons and modulators; and the accelerator sections, including accelerating and feed waveguides, vacuum systems, and supports.

Net conversion efficiency of line power to beam power for single-bunch operation of a conventional SLAC-style linac is considerably less than $1 \%$. As average beam power of $10 \mathrm{MW}$ or more will be required to produce useful luminosities at a colliding linear accelerator facility, efficiencies of acceleration must be improved considerably to avoid unacceptable electric power usage.

Assuming the necessary technological developments, colliding electron and positron linear accelerators might provide, at $2-\mathrm{TeV}$ center-of-mass, a luminosity of $1.5 \times 10^{32} \mathrm{~cm}^{-2} \mathrm{sec}^{-1}$ with an energy spread of $10 \%$ at each of six parallel interaction regions. Such linacs might be used also for electron-proton collisions if a suitable cooled proton source (e.g., cooling ring) could be developed.

D. Advanced Technology Research and Development

Research and development in the technologies of particle beam acceleration, beam control, other accelerator-related systems and instrumentation, and detection systems for experimentation are essential for maintaining the forefront nature of the High Energy Physics Program.

New technologies developed in the past have resulted in enormous increases in accelerator energy and decreases in cost per unit energy in the past fifty years, but the present scale of $R$ and $D$ is relatively small--and certainly not comensurate with its importance. One problem is the reluctance of individuals to comit themselves to tasks whose possible fruition seems quite distant. Another is the lack of suitably trained multi-disciplinary experts. A third may be the mechanisms for supporting accelerator physics. Encouragement to universi- 
ties to expand training in accelerator physics may be needed, and it might be desirable to implement a funding mechanism that would allow laboratories to pursue long-term work with an assurance that such funding was truly an addition to that for more immediate goals, since internal priorities tend to force some curtailment of very long-range activities. Despite these problems, it is encouraging that many people are working on new ideas and that advanced accelerator workshops are held at regular intervals. Calculations and research are being done in the U.S. and abroad on various concepts for obtaining higher accelerating gradients (energy gain per unit of accelerator length). These indicate that accelerator structures can eventually be built to handle gradients up to $200 \mathrm{GeV}$ per kilometer, ten times those now available. This will require a suitable high efficiency, high power source of short-wavelength electromagnetic radiation that can provide a relatively large amount of energy per unit length. Some of the possibilities are as follows:

i. Very high power, very short pulse length, high-frequency klystrons suitable for this purpose may be developed.

ii. A special case of a source of short-wavelength electromag netic radiation is the wakefield of a high energy beam passing through a cavity system. This idea is being pursued theoretically and shows considerable promise and a special simplicity since the wakefield source cavity can be combined with a beam-accelerating cavity within a single structure.

iii. In the two-beam accelerator concept, a high power, low energy electron beam travels parallel to the desired high energy particle beam. Using a principle such as that of the free electron laser, the hi.jh-power, low energy beam radiates its power to the high energy beam, thus providing the acceleration.

iv. A more radical approach is to use the very short wavelength obtainable from a laser. In this case one cannot consider accelerating structures of conventional design; the dimensions are far too small. It appears possible, however, to use a suitable optical grating in place of a conventional cavity. The most extreme case would be replacement of the periodic grating by a periodic plasma, possibly formed over a grating surface, by which gradients as high as $1 \mathrm{TeV}$ per kilometer could theoretically be attained. Such high and obviously desirable gradients can exist only in or near a plasma and not in or near any solid conductor or dielectric.

v. A particularly interesting idea is to expose a plasma to two laser beams of suitably close frequency: The beat frequency between the two lasers can be matched to the natural plasma frequency, to induce a strong periodic and moving charge modulation, which generaises large electrostatic fields that could be used to accelerate suitably injected beams. Accelerating fields as high as $2 \mathrm{TeV}$ per kilometer have been discussed, but great uncertainty remains about the stability 
and energy efficiency of such a mechanism and its suitability for use in a high energy linear collider.

Many other ideas have been suggested. Some of them may not work. Others may work but not have appiication for high energy physics. It is clear, however, that without some such idea, no great further step in energy will be economically possible after the SSC. On the other hand, with gradients of the order of $1 \mathrm{TeV} / \mathrm{km}$ theoretically possible, an accelerator of $100 \mathrm{TeV}$ is not unthinkable. It is thus very important to the future of the field that these ideas be followed up in coming years.

Continued advance in the development of instrumentation and detection systems is the experimental high energy physicist's challenge. These systems provide both the raison d'être for accelerator facilities and the means by which theory can be confirmed by experiment. Support for the development of instrumentation will grow during the next decade, and basic research on new detectors will be increasingly recognized and supported as being fundamental to progress.

E. Funding and the Transition to the Superconducting Super Collider

A funding scenario for the future High Energy Physics Program must be based on specific programatic and budgetary assumptions. It is, of course, not a simple task to predict the future. To get a feeling for what might be possible for High Energy Physics in the future, we take the results of a recent HEPAP study, to be found in "Report of the 1985 High Energy Physics Advisory Panel Study of the U.S. High Energy Physics Program 1985-1995." In the following, in order to emphasize that the details of program and budget in this 10-year period are recomendations offered by HEPAP, the use of "we" is maintained as in the report and refers to the HEPAP study group.

High Energy Physics 1985-1995. We are looking forward to the start of SSC construction in 1988 and an expected completion in 1994 after a 6-year construction period. Here we describe a strong U.S. High Energy Physics Program and show how the transition to the SSC might proceed. We look at "snapshots" of the program at three distinct times: in 1987, before the start of SSC construction; in 1991, during the SSC construction; and finally, in 1995, after the SSC has been completed and its operation for experiments has begun. Obvious$1 y$, there are uncertainties associatd with anticipating the program 10 years in the future in a rapidly moving field such as high energy physics. The following is our expectation from today's vantage point.

\section{Evolution of the Research Program}

The Program in 1987. By this time, the new facilities now under construction will be completed. This will be the start of the rich utilization of all of our facilities. At Fermilab, the Tevatron 1 $\mathrm{P} \overrightarrow{\mathrm{p}}$ collider will be in early operation with one det.ctor. The Tevatron II fixed-target programs will be in full operation. At SLAC, 
the $e^{+} e^{-}$collider, SLC, will be in its initial operation and PEP will be in full operation. The lower energy $e^{+} e^{-}$collider, SPEAR, will be utilized about half-time for high energy physics. At Brookhaven, the AGS will be in full operation. The majority of AGS time will be devoted to high energy physics, with a small portion of the time used by the nuclear physics program studying heavy ion collisions. At Cornell, the newly upgraded $e^{t} e^{-}$collider, CESR, will be fully utilized. There will also be a number of U.S. groups working at non-U.S. facilities. Detectors for the $e^{+} e^{-}$collider, LEP, and the $e^{ \pm} p$ collider, HERA, will be under fabrication, and there should be experiments running at the $e^{+} e^{-}$collider, TRISTAN, and the $\bar{p} p$ collider, the $s \bar{p} p s$. A variety of non-accelerator experiments will be taking data. A vigorous SSC $R$ and $D$ program in advance of construction will be in progress, both on the accelerator systems and the associated detectors. There will also be activity in advanced accelerator $R$ and $D$.

The Program in 1991. By this time, we expect the SSC will be in the middle of its construction period. The construction of detectors for the SSC will be in full swing. At Fermilab, the collider will operate with both major detectors, and the full complement of fixedtarget facilities will be utilized, both for experiments and for providing test beams for detector development. At SLAC, the SLC will be in full operation. At Brookhaven, a high intensity AGS with a completed Booster will be utilized partially by the High Energy Physics Program and partially by the Nuclear Physics Heavy Ion Program. At Corne 11, CESR will still be operating. There will also be U.S. groups taking data in experiments at LEP and TRISTAN. Experiments at HERA, with U.S. participation, will be starting up. A variety of nonaccelerator experiments will be taking data. The activity in advanced accelerator $R$ and $D$ will be continuing.

The Program in 1995. By this time, we expect that the SSC will be in its early stages of operation with at least a partial complement of detectors. At Fermilab, we expect that the collider will be running with a mature program; the fixed-target facilities will be operating for an experimental program at a reduced level in addition to supplying test beams for new detector development. The SLC at SLAC will still be in operation. The AGS at Brookhaven is expected to be almost fully occupied as an injector for the heavy ion collider, RHIC, and as a fixed-target facility for the Nuclear Physics Program and is not expected to be utilized significantly by the High Energy Physics Program. U.S. groups will probably still be involved at LEP II and HERA, but the TRISTAN involvement is expected to be winding down. A variety of non-accelerator experiments will be taking data. We anticipate a vigorous program in advanced accelerator $R$ and $D$ at this time. It will be difficult to maintain a forefront U.S. program if the operation of the SSC is delayed beyond this time.

\section{Funding Required for the High Energy Physics Program}

An estimate has beem compiled for a minimum budget required to carry out the program described here. For the pre-SSC program in FY 
1987 , these estimates are $\$ 435$ million for operating, $\$ 90$ million for equipment, $\$ 70$ million for construction (for a total of $\$ 595$ million non-SSC related) and $\$ 65$ million for SSC $R$ and $D$, for a total High Energy Physics budget from the DOE of $\$ 660$ million. An additional $\$ 55$ million is required from the National Science Foundation (all in FY 1987 dollars). For comparison, the FY 1985 High Energy Physics budget (converted to FY 1987 dollars) was $\$ 400$ million in operating, $\$ 66$ million in equipment, and $\$ 129$ million in construction (for a total of $\$ 595$ million non-SSC related) and $\$ 23$ million for SSC $R$ and $D$, for a total DOE budget of $\$ 618$ million, with an additional $\$ 46$ million from the NSF. We note that while the total non-SSC-related funds needed for FY 1987 are essentially the same as in FY 1985, the operating funds are up and the construction funds are down relative to FY 1985 . This is necessary for the following reasons. Two of the major accelerator facilities of the High Energy Physics Program will be coming into full operation by 1987 . These unique state-of-the-art accelerators (SLC and Tevatron) are not only the mainstay of the High Energy Physics Program but also prototypes for future higher energy accelerators. It is essential that incremental operating funds be provided for these facilities.

With these assumptions, the projection of the DOE High Energy Physics budget to FY 1995 is given in Table XIII. Here the SSC construction, equipment, and operating costs from the SSC Reference Designs Study have been used. It can be seen that a substantial pulse of incremental funding over the $\mathrm{FY} 1987$ level is required for the SSC construction for the FY 1988-1993 period. After this period, the funding required for the program, including the operation of the SSC, returns to within about $10 \%$ of the FY 1985 level. During the SSC construction period, the non-SSC part of the budget declines, reaching a reduced level of about $\$ 400$ million by FY 1995. The SSC operating costs are expected to be $\$ 215$ million with an additional $\$ 50$ million in equipment funds and $\$ 15$ million in accelerator improvement (AIP/GPP) funds, for a total High Energy Physics budget from the DOE in FY 1995 of about $\$ 680$ million (in FY 1987 dollars).

\section{Scientific Manpower}

Current estimates indicate that the U.S. scientific manpower ( $P h . D$. physicists or equivalent) working in high energy physics at the present time consists of about 1150 experimentalists, 750 theorists, and 330 accelerator scientists. The number of experimentalists now occupied in carrying out the present program is not a bad match to the requirement of the program during the SSC construction period and the period after the start of SSC operation. We do, however, anticipate a scarcity of accelerator physicists during the SSC construction period. A need for a 20 to $30 \%$ increase in the number of accelerator scientists is anticipated. A vigorous program of training present high energy physics experimentalists and theorists in accelerator science is of high importance in the near future. In addition, efforts should be made to make a career in accelerator physics attractive to young physicists entering the field of high energy physics. We anticipate a flow of experimentalists and accelerator 
Table XIII Projected High Energy Physics Budgets 1985-1995 (in units of millions of FY 1987 dollars)

\begin{tabular}{|c|c|c|c|c|}
\hline & EY 85 & FY 87 & FY 91 & FY 95 \\
\hline \multicolumn{5}{|l|}{ Non-SSC Program } \\
\hline Operating* & 400 & 435 & 410 & 350 \\
\hline Equipment & 66 & 90 & 70 & 40 \\
\hline Construction & 129 & 70 & 20 & 10 \\
\hline Total Non-SSC & 595 & 595 & 500 & 400 \\
\hline \multicolumn{5}{|l|}{ SSC Program } \\
\hline$R$ and $D$ & 23 & 65 & 40 & 0 \\
\hline Construction & & & 700 & 15 \\
\hline Equipment & & & 130 & 50 \\
\hline Operating & & & 30 & 215 \\
\hline Total SSC & 23 & 65 & 900 & 280 \\
\hline Total DOE Program & $618 * *$ & 660 & 1400 & 680 \\
\hline NSF & 46 & 55 & 48 & 48 \\
\hline
\end{tabular}

* Includes University budgets, part of which will be spent on SSC experiments. $* * 546$ in FY 85 s.

physicists from the present program to the program including the SSC. We expect that in addition to the U.S. physicists, there will be a substantial number of non-U.S. experimentalists working at the SSC. This analysis shows that the existing high energy physics manpower, with an anticipaced $10 \%$ growth over the next decade, can accomplish the program described here.

In sumary, the major points are the following:

i. A budget slightly above the FY 1985 level would be adequate to permit effective research with the new facilities at present being completed and would allow the needed $R$ and $D$ for the $S S C$ in the period prior to the initiation of construction.

ii. During the SSC construction phase, there will be needed an increment, or pulse, in capital funds for construction and detectors.

iii. After the SSC is in operation, a productive progran can be achieved with a funding level in constant-year dollars not much larger than that prior to SSC construction. This is possible because some of the present facilities will. have reached maturity by then and there can be an orderly transition from these activities to research on the SSC.

These points are shown schematically in Figure 25, with budget figures given in constant 1984 dollars. 


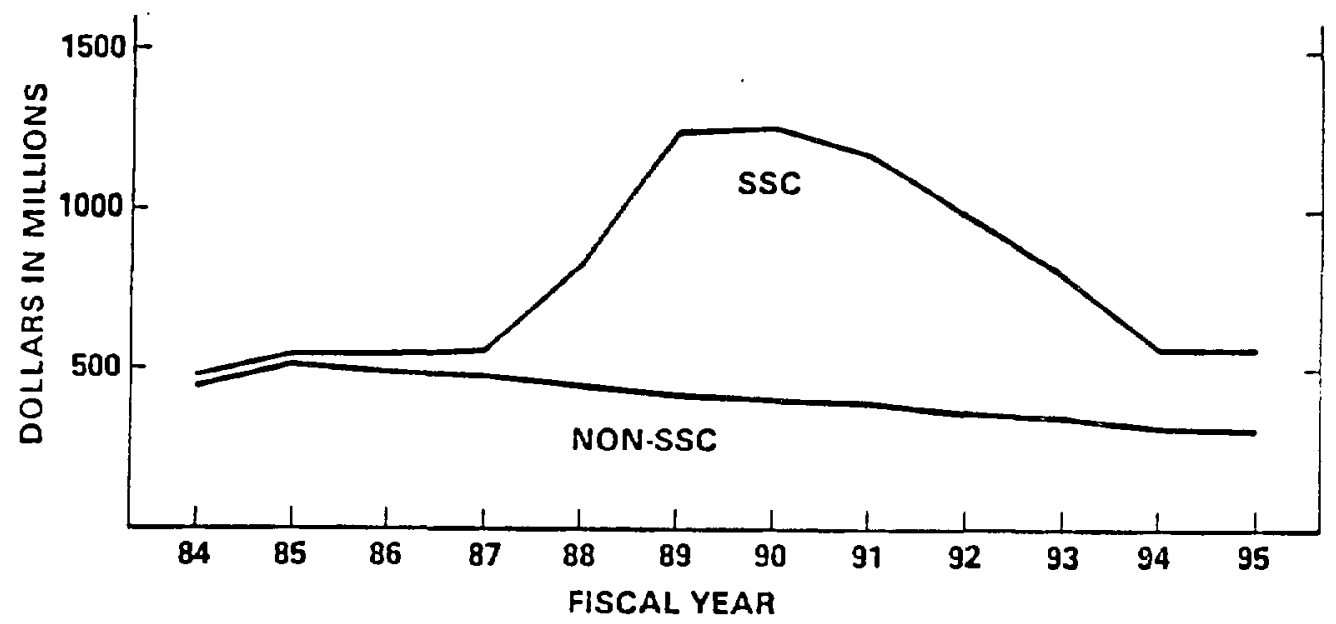

Figure 25. High energy physics funding scenario (FY 84 dollars) for the DOE-supported U.S. High Energy Physics Program. Indicated is the base program (including operation of major facilities in existence or under construction, advanced technology $R$ and $D$, and the facility user groups at universities and laboratories) and the funding for the SSC including preconstruction $R$ and $D, R$ and $D$ in support of construction, construction costs, pre-operating costs, and the design and fabrication of the initial complement of detectors. 